\title{
Hydrazonyl Radical-Participated Tandem Reaction: A Strategy for the Synthesis of Pyrazoline-Functionalized Oxindoles
}

Xiao-Yong Duan, Xiu-Long Yang, Pan-Pan Jia, Man Zhang, and Bing Han* State Key Laboratory of Applied Organic Chemistry, Lanzhou University, Lanzhou, Gansu, 730000, People's Republic of China

hanb@1zu.edu.cn

\section{Supporting Information}

- Contents page

- General

- Methods for the synthesis of ketones 2

- Methods for the synthesis of substituted hydrazones 4

- Methods for the synthesis of substituted amides 4

- General experimental procedure 5

- References 5

- Analytical data for new substrates $\quad 6$

- Analytical data for products $\quad 8$

- Copies of ${ }^{1} \mathrm{H}$ and ${ }^{13} \mathrm{C}$ spectra of new substrates 26

- Copies of ${ }^{1} \mathrm{H}$ and ${ }^{13} \mathrm{C}$ spectra of products 38 


\section{General:}

All reagents were purchased from commercial suppliers and used without further purification. Flash chromatography was carried out with silica gel (200-300 mesh). Analytical TLC was performed with silica gel GF254 plates, and the products were visualized by UV detection. ${ }^{1} \mathrm{H}$ NMR and ${ }^{13} \mathrm{C}$ NMR $(400 \mathrm{MHz}$ and $100 \mathrm{MHz}$, respectively) spectra were recorded in $\mathrm{CDCl}_{3}$. Chemical shifts $(\delta)$ are reported in ppm using TMS as internal standard and spin-spin coupling constants $(J)$ are given in $\mathrm{Hz}$. The high resolution mass spectra (HRMS) were measured on a Bruker Daltonics APEX II $47 \mathrm{e}$ spectrometer by ESI. Data collections for crystal structure were performed at room temperature (293 K) using Mo $K \alpha$ radiation on a Bruker APEXII diffractometer. Integration of the frames and data reduction was carried out using SAINT. The structure was solved by direct methods using SHELXS-97.

\section{Methods for the synthesis of ketones: ${ }^{[1]}$}

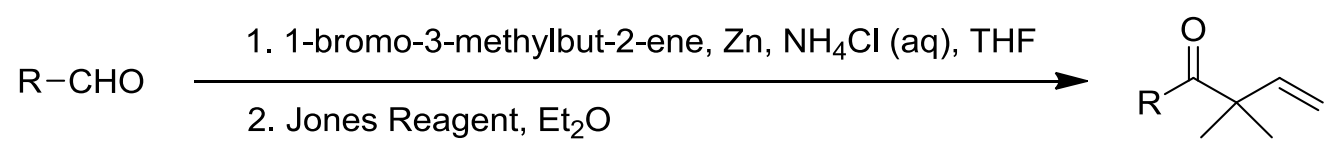

(1) A round bottomed flask charged with a solution of the 3-bromo-2-methylprop1-ene (2.0 equiv) and the aldehyde (1.0 equiv) in $\mathrm{THF} / \mathrm{H}_{2} \mathrm{O}(1: 1)$ was kept at $0{ }^{\circ} \mathrm{C}$ with stirring. The $\mathrm{Zn}$ powder (2.0 equiv) was slowly added to the solution and the resulting suspension was stirred at RT for $24 \mathrm{~h}$ as monitored by TLC. $1 \mathrm{~N}$ hydrochloride solution was added at $0{ }^{\circ} \mathrm{C}$. The THF layer was separated from the aqueous layer, which was extracted with diethyl ether for 3 times. The combined organic layers were washed with brine, dried over $\mathrm{Na}_{2} \mathrm{SO}_{4}$, filtered and concentrated in vacuo. The crude product was directly used in the next step without further purification.

(2) To the previous prepared mixture was added a solution of Jones reagent (12 $\mathrm{mL})$ and $\mathrm{H}_{2} \mathrm{O}(12 \mathrm{~mL})$ at $0{ }^{\circ} \mathrm{C}$. The mixture was stirred at $0{ }^{\circ} \mathrm{C}$ for $1-2 \mathrm{~h}, \mathrm{H}_{2} \mathrm{O}(50 \mathrm{~mL})$ was added, and extracted with $\mathrm{Et}_{2} \mathrm{O}(300 \mathrm{~mL})$. The organic extracts were washed with 
brine $(30 \mathrm{~mL})$, dried $\left(\mathrm{Na}_{2} \mathrm{SO}_{4}\right)$ and concentrated in vacuo. Purification of the residue was performed by silica gel column chromatography (hexanes-EtOAc 40:1) to give product in about $75 \%$ yield.

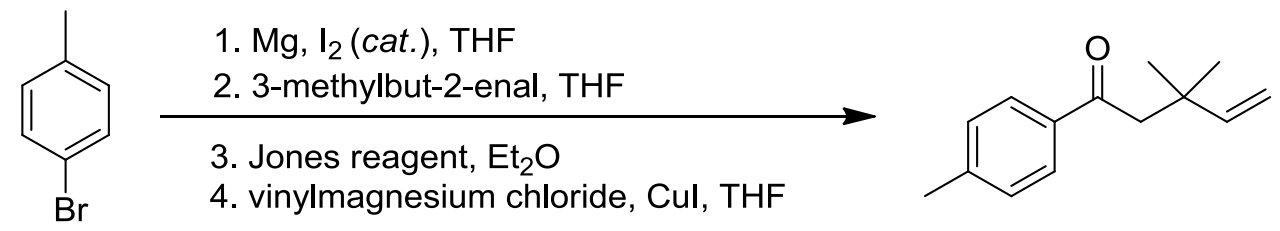

(1) Under Ar atmosphere, a solution of 1-bromo-4-methylbenzene (20 mmol) and THF (20 mL) was slowly added to a mixture of Mg (24 mmol), $\mathrm{I}_{2}$ (cat.) and THF (20 $\mathrm{mL})$. Then the mixture was stirred at reflux for $6 \mathrm{~h}$.

(2) A solution of 3-methylbut-2-enal (22 mmol) and THF (20 mL) was added to the previous prepared solution of Grignard reagent. The mixture was stirred at RT for $2 \mathrm{~h}$ as monitored by TLC. The reaction was quenched adding saturated $\mathrm{NH}_{4} \mathrm{Cl}$, and extracted with $\mathrm{Et}_{2} \mathrm{O}$. The organic extracts were washed with brine, dried $\left(\mathrm{Na}_{2} \mathrm{SO}_{4}\right)$ and concentrated to $100 \mathrm{~mL}$ in vacuo.

(3) To the previous prepared mixture was added a solution of Jones reagent (12 mL) and $\mathrm{H}_{2} \mathrm{O}(12 \mathrm{~mL})$ at $0{ }^{\circ} \mathrm{C}$. The mixture was stirred at $0{ }^{\circ} \mathrm{C}$ for $1-2 \mathrm{~h}, \mathrm{H}_{2} \mathrm{O}(50 \mathrm{~mL})$ was added, and extracted with $\mathrm{Et}_{2} \mathrm{O}(300 \mathrm{~mL})$. The organic extracts were washed with brine $(30 \mathrm{~mL})$, dried $\left(\mathrm{Na}_{2} \mathrm{SO}_{4}\right)$ and concentrated in vacuo. Purification of the residue was performed by silica gel column chromatography (hexanes-EtOAc 40:1) to give product in $75 \%$ yield $(2.61 \mathrm{~g}, 15 \mathrm{mmol})$.

(4) Under Ar atmosphere, a mixture of the previous prepared 3-methyl-1-(p-tolyl)but-2-en-1-one (10 mmol), $\mathrm{CuI}(1 \mathrm{mmol})$ and THF $(20 \mathrm{~mL})$ was cooled to $-40{ }^{\circ} \mathrm{C}$, and a solution of vinylmagnesium chloride $(12 \mathrm{mmol})$ was added to the mixture. Then the mixture was stirred at RT overnight. The reaction was quenched adding saturated $\mathrm{NH}_{4} \mathrm{Cl}$, and extracted with $\mathrm{Et}_{2} \mathrm{O}$. The organic extracts dried $\left(\mathrm{Na}_{2} \mathrm{SO}_{4}\right)$ and concentrated in vacuo. Purification of the residue was performed by silica gel column chromatography (hexanes-EtOAc 100:1) to give product in 60\% yield (1.21 g, 6 mmol). 


\section{Methods for the synthesis of substituted hydrazones: ${ }^{[1]}$}

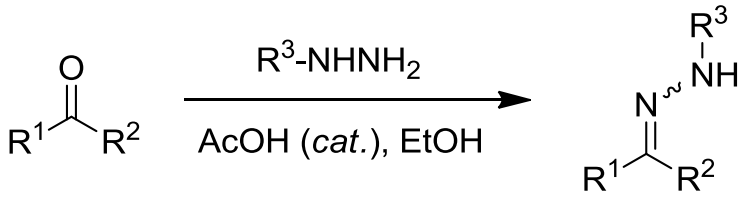

$$
\begin{aligned}
& \mathrm{R}^{3}=\text { phenyl, acetyl, tosyl }
\end{aligned}
$$

To a solution of the ketone (1.0 equiv) in anhydrous ethanol was added $N$-substituted hydrazines (1.5 equiv) and acetic acid (0.2 equiv). The mixture was stirred at reflux and monitored by TLC. Then the mixture was directly concentrated in vacuo. The crude material was purified by flash chromatography on silica gel to afford the hydrazone in $60-70 \%$ yield.

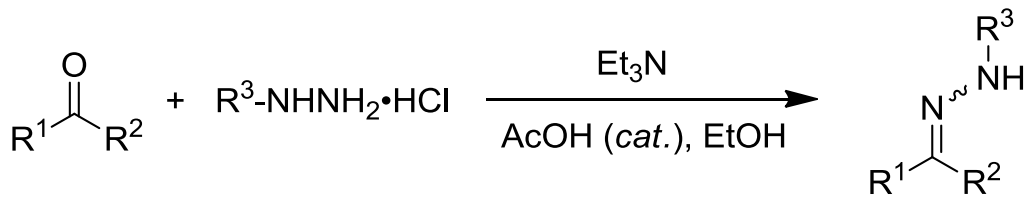

$$
\begin{aligned}
& \mathrm{R}^{3}=\text { alkyl, aryl }
\end{aligned}
$$

To a solution of the ketone $(1.0 \mathrm{mmol})$ in $2 \mathrm{~mL}$ of anhydrous ethanol and acetic acid $(0.2 \mathrm{~mL})$ was added a solution of $N$-substituted hydrazine hydrochloride $(1.5 \mathrm{mmol})$ and $\mathrm{Et}_{3} \mathrm{~N}(0.2 \mathrm{~mL}, 1.5 \mathrm{mmol})$ in $3 \mathrm{~mL}$ of ethanol. The reaction mixture was stirred overnight at reflux and monitored by TLC. Then the mixture was directly concentrated in vacuo. The crude material was purified by flash chromatography on silica gel to afford the hydrazone in $45-60 \%$ yield.

\section{Methods for the synthesis of substituted amide: $:^{[2]}$}

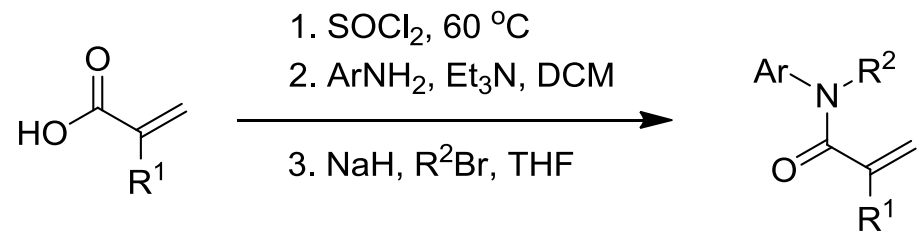

(1) A mixture of acrylic acid (12 mmol) and $\mathrm{SOCl}_{2}(14 \mathrm{mmol})$ was stirred at $60{ }^{\circ} \mathrm{C}$ for $2 \mathrm{~h}$.

(2) A mixture of arylamine (10 mmol), $\mathrm{Et}_{3} \mathrm{~N}(12 \mathrm{mmol})$ and DCM (20 mL) was stirred 
at $0{ }^{\circ} \mathrm{C}$, then a solution of the previous prepared acyl chloride and DCM $(10 \mathrm{~mL})$ was added slowly into the mixture. And the reaction mixture was stirred at RT and monitored by TLC. The reaction was quenched adding saturated $\mathrm{NaHCO}_{3}$, and extracted with DCM. The organic extracts dried $\left(\mathrm{Na}_{2} \mathrm{SO}_{4}\right)$ and concentrated in vacuo. Purification of the residue was performed by silica gel column chromatography (hexanes-EtOAc 5:1) to give product in about 70\% yield.

(3) A mixture of acrylamide $(8 \mathrm{mmol})$, and THF $(20 \mathrm{~mL})$ was stirred at $0{ }^{\circ} \mathrm{C}$, NaH $(10$ mmol) was added slowly to the mixture. Then the reaction mixture was stirred at $0{ }^{\circ} \mathrm{C}$ for $30 \mathrm{~min}$, and $\mathrm{R}^{2} \mathrm{Br}(10 \mathrm{mmol})$ was added to the mixture. At last, the mixture was stirred at RT for $2 \mathrm{~h}$ and monitored by TLC. The reaction was quenched by adding $\mathrm{H}_{2} \mathrm{O}$, and extracted with $\mathrm{Et}_{2} \mathrm{O}$. The organic extracts dried $\left(\mathrm{Na}_{2} \mathrm{SO}_{4}\right)$ and concentrated in vacuo. Purification of the residue was performed by silica gel column chromatography (hexanes-EtOAc 10:1) to give product in about $80 \%$ yield.

\section{General experimental procedure:}

A mixture of $N$-aryl acrylamide $1(0.5 \mathrm{mmol})$, hydrazone $2(1.0 \mathrm{mmol})$ and DTBP (3.25 mmol) was placed in a $10 \mathrm{~mL}$ Schlenk tube and stirred at $100{ }^{\circ} \mathrm{C}$ under argon for $72 \mathrm{~h}$. When the starting materials were consumed completely as monitored by TLC, the reaction mixture was isolated by silica gel column chromatography to give the product in $42-94 \%$ yield. The identity and purity of the product was confirmed by ${ }^{1} \mathrm{H}$ and ${ }^{13} \mathrm{C}$ NMR spectroscopic analysis.

\section{Reference:}

1. X. Y. Duan, X. L. Yang, R. Fang, X. X. Peng, W. Yu and B. Han, J. Org. Chem., 2013, 78, 10692;

2. L. N. Guo, H. Wang and X. H. Duan, Chem. Commun., 2013, 49, 7540; 


\section{Analytical data for new substrates:}<smiles>C=C(C)C(=O)N(C)c1ccc(-c2ccccc2)cc1</smiles>

\section{$N-([1,1$ '-biphenyl]-4-yl)-N-methylmethacrylamide (1i)}

Yellow solid, mp 94-95 ${ }^{\circ} \mathrm{C} ; \mathrm{R}_{f}=0.55$ (hexanes/ethyl acetate 5:1, twice); ${ }^{1} \mathrm{H}$ NMR $\left(400 \mathrm{MHz}, \mathrm{CDCl}_{3}\right): \delta 7.59-7.55(\mathrm{~m}, 4 \mathrm{H}), 7.44(\mathrm{t}, J=8.0 \mathrm{~Hz}, 2 \mathrm{H}), 7.35(\mathrm{t}, J=7.2 \mathrm{~Hz}$, $1 \mathrm{H}), 7.20(\mathrm{~d}, J=8.4 \mathrm{~Hz}, 2 \mathrm{H}), 5.07(\mathrm{~s}, 1 \mathrm{H}), 5.05(\mathrm{~s}, 1 \mathrm{H}), 3.38(\mathrm{~s}, 3 \mathrm{H}), 1.81(\mathrm{~s}, 3 \mathrm{H}) ;{ }^{13} \mathrm{C}$ NMR (100 MHz, $\left.\mathrm{CDCl}_{3}\right): \delta$ 171.9, 143.7, 140.6, 139.9, 139.6, 128.8, 127.7, 127.5, 126.9, 126.6, 119.4, 37.6, 20.3; ESI-HRMS: $\mathrm{m} / z$ Calcd for $\mathrm{C}_{17} \mathrm{H}_{17} \mathrm{NO}+\mathrm{H}^{+}: 252.1383$, found 252.1381 .<smiles>C=CC(C)(C)/C(=N\NC)c1ccccc1</smiles>

\section{1-(2, 2-dimethyl-1-phenylbut-3-en-1-ylidene)-2-methylhydrazine (2f)}

Yellow oil; $\mathrm{R}_{f}=0.40$ (hexanes/ethyl acetate 100:1); ${ }^{1} \mathrm{H}$ NMR $\left(400 \mathrm{MHz}, \mathrm{CDCl}_{3}\right): \delta$ $7.41-7.38(\mathrm{~m}, 2 \mathrm{H}), 7.35-7.40(\mathrm{~m}, 1 \mathrm{H}), 6.77(\mathrm{dd}, J=6.8 \mathrm{~Hz}, J=1.6 \mathrm{~Hz}, 2 \mathrm{H}), 5.98(\mathrm{dd}$, $J=17.6 \mathrm{~Hz}, J=10.4 \mathrm{~Hz}, 1 \mathrm{H}), 4,99-4.90(\mathrm{~m}, 2 \mathrm{H}), 4.39$ (br, 1H), 2.81 (s, 3H), $1.22(\mathrm{~s}$, $6 \mathrm{H}) ;{ }^{13} \mathrm{C} \mathrm{NMR}\left(100 \mathrm{MHz}, \mathrm{CDCl}_{3}\right): \delta 154.1,145.7,134.3,128.6,128.5,128.0,111.4$, 43.5, 37.7, 25.5; ESI-HRMS: $m / z$ Calcd for $\mathrm{C}_{13} \mathrm{H}_{18} \mathrm{~N}_{2}+\mathrm{H}^{+}$: 203.1543, found 203.1540.<smiles>C=CC(C)(C)/C(=N\NC(C)=O)c1ccccc1</smiles>

\section{$N^{\prime}$-(2, 2-dimethyl-1-phenylbut-3-en-1-ylidene) acetohydrazide (2g)}

Yellow oil; $\mathrm{R}_{f}=0.35$ (hexanes/ethyl acetate 10:1); ${ }^{1} \mathrm{H}$ NMR (400 $\left.\mathrm{MHz}, \mathrm{CDCl}_{3}\right): \delta$ 7.90 (br, 1H), 7.44-7.39 (m, 3H), 7.01 (dd, $J=7.6 \mathrm{~Hz}, J=1.6 \mathrm{~Hz}, 2 \mathrm{H}), 5.92$ (dd, $J=$ $18.8 \mathrm{~Hz}, J=10.8 \mathrm{~Hz}, 1 \mathrm{H}), 5.06-4.96(\mathrm{~m}, 2 \mathrm{H}), 2.30(\mathrm{~s}, 3 \mathrm{H}), 1.26(\mathrm{~s}, 6 \mathrm{H}) ;{ }^{13} \mathrm{C} \mathrm{NMR}$ $\left(100 \mathrm{MHz}, \mathrm{CDCl}_{3}\right): \delta 172.8,158.3,144.2,131.9,129.1,129.0,128.0,112.8,44.2$, 
25.2, 20.4; ESI-HRMS: $m / z$ Calcd for $\mathrm{C}_{14} \mathrm{H}_{18} \mathrm{~N}_{2} \mathrm{O}+\mathrm{H}^{+}$: 231.1492, found 231.1489.<smiles>C=CC(C)(C)C(=NNS(=O)(=O)c1ccc(C)cc1)c1ccccc1</smiles>

\section{$N^{\prime}$-(2,2-dimethyl-1-phenylbut-3-en-1-ylidene)-4-methylbenzenesulfonohydrazide} (2h)

White solid, mp $102-103{ }^{\circ} \mathrm{C} ; \mathrm{R}_{f}=0.35$ (hexanes/ethyl acetate 5:1); ${ }^{1} \mathrm{H}$ NMR (400 $\left.\mathrm{MHz}, \mathrm{CDCl}_{3}\right): \delta 7.78(\mathrm{~d}, J=8.4 \mathrm{~Hz}, 2 \mathrm{H}), 7.38(\mathrm{t}, J=3.0 \mathrm{~Hz}, 3 \mathrm{H}), 7.33(\mathrm{~d}, J=8.0 \mathrm{~Hz}$, 2H), $6.96(\mathrm{br}, 1 \mathrm{H}), 6.81(\mathrm{dd}, J=5.2 \mathrm{~Hz}, J=2.8 \mathrm{~Hz}, 2 \mathrm{H}), 5.73(\mathrm{dd}, J=17.6 \mathrm{~Hz}, J=$ $10.4 \mathrm{~Hz}, 1 \mathrm{H}), 4.97-4.82(\mathrm{~m}, 2 \mathrm{H}), 2.46$ (s, 3H), $1.16(\mathrm{~s}, 6 \mathrm{H}) ;{ }^{13} \mathrm{C}$ NMR (100 MHz, $\left.\mathrm{CDCl}_{3}\right): \delta 162.9,143.9,143.8,135.3,131.4,129.34,129.31,129.1,127.9,127.6$, 113.1, 44.4, 25.1, 21.6; ESI-HRMS: $m / z$ Calcd for $\mathrm{C}_{19} \mathrm{H}_{22} \mathrm{~N}_{2} \mathrm{O}_{2} \mathrm{~S}+\mathrm{H}^{+}: 343.1475$, found 343.1474 .<smiles>C=CC(C)(C)C(=NNc1ccccc1)c1ccc2ccccc2c1</smiles>

\section{1-(2, 2-dimethyl-1-(naphthalen-2-yl) but-3-en-1-ylidene)-2-phenylhydrazine (2m)}

Yellow oil; $\mathrm{R}_{f}=0.55$ (hexanes/ethyl acetate 40:1); ${ }^{1} \mathrm{H}$ NMR (400 $\left.\mathrm{MHz}, \mathrm{CDCl}_{3}\right): \delta$ 7.92-7.84 (m, 3H), $7.60(\mathrm{~s}, 1 \mathrm{H}), 7.55-7.51$ (m, 2H), 7.21-7.13 (m, 3H), 6.91-6.86 (m, $3 \mathrm{H}), 6.77-6.74(\mathrm{~m}, 1 \mathrm{H}), 6.12-6.04(\mathrm{~m}, 1 \mathrm{H}), 5.05-4.96(\mathrm{~m}, 2 \mathrm{H}), 1.36(\mathrm{~s}, 6 \mathrm{H}) ;{ }^{13} \mathrm{C}$ NMR (100 MHz, $\left.\mathrm{CDCl}_{3}\right): \delta$ 152.0, 145.6, 145.3, 133.1, 133.0, 130.9, 129.0, 128.7, 128.2, 128.1, 127.8, 126.7, 126.5, 119.4, 112.6, 112.1, 44.3, 25.7; ESI-HRMS: $\mathrm{m} / \mathrm{z}$ Calcd for $\mathrm{C}_{22} \mathrm{H}_{22} \mathrm{~N}_{2}+\mathrm{H}^{+}$: 315.1856, found 315.1851. 


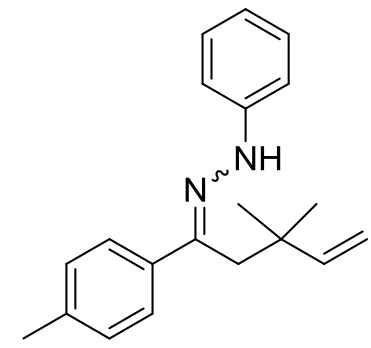

1-(3, 3-dimethyl-1-(p-tolyl) pent-4-en-1-ylidene)-2-phenylhydrazine (2q)

Yellow oil; $\mathrm{R}_{f}=0.60$ (hexanes/ethyl acetate 40:1); ${ }^{1} \mathrm{H}$ NMR (400 MHz, $\left.\mathrm{CDCl}_{3}\right): \delta$ 7.27 (br, 1H), 7.65 (d, $J=8.4 \mathrm{~Hz}, 2 \mathrm{H}), 7.45$ (br, 1H), 7.28-7.21 (m, 6H), 7.19-7.15 (m, 7H), $7.09(\mathrm{~d}, J=8.2 \mathrm{~Hz}, 2 \mathrm{H}), 7.95(\mathrm{~d}, J=8.0 \mathrm{~Hz}, 1 \mathrm{H}), 6.85(\mathrm{t}, J=7.2 \mathrm{~Hz}, 1 \mathrm{H})$, $6.77(\mathrm{t}, J=7.2 \mathrm{~Hz}, 1 \mathrm{H}), 5.97-5.90(\mathrm{~m}, 1 \mathrm{H}), 5.82-5.75(\mathrm{~m}, 1 \mathrm{H}), 5.12-5.00(\mathrm{~m}, 2 \mathrm{H})$, 4.86-4.76 (m, 2H), 2.76 (s, 2H), 2.63 (s, 2H), 2.39 (s, 3H), $2.36(\mathrm{~s}, 3 \mathrm{H}), 1.09$ (s, 6H), $1.01(\mathrm{~s}, 6 \mathrm{H}) ;{ }^{13} \mathrm{C} \mathrm{NMR}\left(100 \mathrm{MHz}, \mathrm{CDCl}_{3}\right): \delta 148.2,148.1,145.3,143.6,138.5$, $137.59,137.57,132.5,129.8,129.2,129.1,128.9,127.8,126.1,119.9,119.3,113.0$, 112.6, 111.5, 110.0, 50.4, 38.3, 38.1, 37.6, 28.0, 27.2, 21.3, 21.1; ESI-HRMS: $\mathrm{m} / \mathrm{z}$ Calcd for $\mathrm{C}_{20} \mathrm{H}_{24} \mathrm{~N}_{2}+\mathrm{H}^{+}$: 293.2012, found 293.2010.

\section{Analytical data for products:}

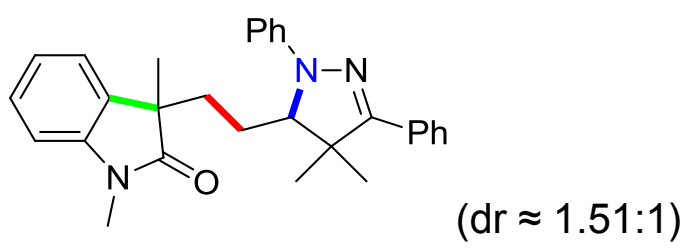

3-(2-(4, 4-dimethyl-1, 3-diphenyl-4, 5-dihydro-1H-pyrazol-5-yl) ethyl)-1, 3dimethylindolin-2-one (3a)

Yellow oil; (192 mg, 88\% yield); $\mathrm{R}_{f}=0.45$ (hexanes/acetone 5:1, twice); ${ }^{1} \mathrm{H}$ NMR $\left(400 \mathrm{MHz}, \mathrm{CDCl}_{3}\right): \delta 7.72(\mathrm{~d}, J=8.0 \mathrm{~Hz}, 2 \mathrm{H}), 7.66(\mathrm{~d}, J=8.0 \mathrm{~Hz}, 2 \mathrm{H}), 7.39-7.29(\mathrm{~m}$, 7H), 7.23-7.14 (m, 7H), 7.07-7.01 (m, 3H), 6.91-6.73 (m, 6H), 6.45 (d, J = 7.2 Hz, $1 \mathrm{H}), 3.82(\mathrm{~d}, J=6.0 \mathrm{~Hz}, 1 \mathrm{H}), 3.61(\mathrm{~d}, J=8.0 \mathrm{~Hz}, 1 \mathrm{H}), 3.14(\mathrm{~s}, 3 \mathrm{H}), 3.13(\mathrm{~s}, 3 \mathrm{H}), 2.01$ $(\mathrm{td}, J=6.0 \mathrm{~Hz}, J=4.0 \mathrm{~Hz}, 1 \mathrm{H}), 1.82(\mathrm{td}, J=6.0 \mathrm{~Hz}, J=4.0 \mathrm{~Hz}, 1 \mathrm{H}), 1.76(\mathrm{td}, J=8.0$ $\mathrm{Hz}, J=4.0 \mathrm{~Hz}, 1 \mathrm{H}), 1.63-1.52(\mathrm{~m}, 3 \mathrm{H}), 1.42$ (s, 3H), 1.40 (s, 3H), 1.37 (s, 3H), 1.33 $(\mathrm{s}, 3 \mathrm{H}), 1.31(\mathrm{~s}, 3 \mathrm{H}), 1.29-1.25(\mathrm{~m}, 2 \mathrm{H}), 1.20(\mathrm{~s}, 3 \mathrm{H}) ;{ }^{13} \mathrm{C} \mathrm{NMR}\left(100 \mathrm{MHz}, \mathrm{CDCl}_{3}\right): \delta$ $180.4,180.0,155.6,155.2,144.8,144.5,143.4,143.2$, 133.4, 133.1, 132.58, 132.57, 
129.1, 128.9, 128.43, 128.35, 128.31, 128.3, 128.0, 127.5, 126.9, 122.8, 122.6, 122.4, 122.3, 119.5, 119.1, 114.4, 114.2, 108.2, 107.7, 72.1, 71.3, 50.0, 49.2, 48.3, 48.2, 34.9, 32.8, 28.09, 28.06, 26.2, 26.1, 24.6, 23.8, 22.3, 22.1, 19.5, 19.2; ESI-HRMS: $\mathrm{m} / \mathrm{z}$ Calcd for $\mathrm{C}_{29} \mathrm{H}_{31} \mathrm{~N}_{3} \mathrm{O}+\mathrm{H}^{+}$: 438.2540, found 438.2532.

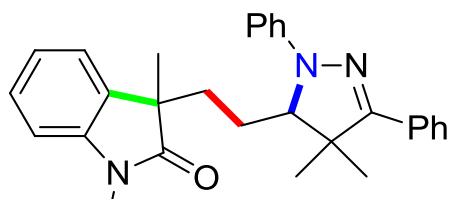

$$
\mathrm{Bn}^{\prime} \quad(\mathrm{dr} \approx 1.79: 1)
$$

1-benzyl-3-(2-(4, 4-dimethyl-1, 3-diphenyl-4, 5-dihydro-1H-pyrazol-5-yl) ethyl)-3methylindolin-2-one $(3 b)$

Yellow oil; (200 mg, 78\% yield); $\mathrm{R}_{f}=0.55$ (hexanes/acetone 5:1, twice); ${ }^{1} \mathrm{H}$ NMR (400 MHz, $\left.\mathrm{CDCl}_{3}\right): \delta 7.73(\mathrm{~d}, J=6.8 \mathrm{~Hz}, 2 \mathrm{H}), 7.66(\mathrm{~d}, J=6.8 \mathrm{~Hz}, 2 \mathrm{H}), 7.40-7.30(\mathrm{~m}$, 9H), 7.29-7.10 (m, 13H), 7.06-7.00 (m, 5H), $6.93(\mathrm{~d}, J=8.0 \mathrm{~Hz}, 1 \mathrm{H}), 6.85(\mathrm{t}, J=7.2$ $\mathrm{Hz}, 1 \mathrm{H}), 6.79$ (t, $J=7.2 \mathrm{~Hz}, 2 \mathrm{H}), 6.68-6.64(\mathrm{~m}, 2 \mathrm{H}), 6.49$ (d, $J=6.8 \mathrm{~Hz}, 1 \mathrm{H}), 4.83$ $(\mathrm{dd}, J=36.0 \mathrm{~Hz}, J=15.6 \mathrm{~Hz}, 4 \mathrm{H}), 3.81(\mathrm{~d}, J=5.2 \mathrm{~Hz}, 1 \mathrm{H}), 3.62(\mathrm{dd}, J=7.6 \mathrm{~Hz}, J=$ $3.2 \mathrm{~Hz}, 1 \mathrm{H}), 2.07(\mathrm{td}, J=13.0 \mathrm{~Hz}, J=5.0 \mathrm{~Hz}, 1 \mathrm{H}), 1.92(\mathrm{td}, J=13.2 \mathrm{~Hz}, J=4.3 \mathrm{~Hz}$, $1 \mathrm{H}), 1.79(\mathrm{td}, J=12.6 \mathrm{~Hz}, J=3.6 \mathrm{~Hz}, 1 \mathrm{H}), 1.65(\mathrm{td}, J=13.2 \mathrm{~Hz}, J=3.6 \mathrm{~Hz}, 1 \mathrm{H})$, 1.60-1.42 (m, 2H), 1.41 (s, 3H), 1.39 (s, 3H), 1.38 (s, 3H), 1.35 (s, 3H), 1.32-1.31 (m, 1H), $1.30(\mathrm{~s}, 3 \mathrm{H}), 1.29-1.27(\mathrm{~m}, 4 \mathrm{H}) ;{ }^{13} \mathrm{C} \mathrm{NMR}\left(100 \mathrm{MHz}, \mathrm{CDCl}_{3}\right): \delta 180.4,180.1$, $155.5,155.1,144.8,144.5,142.4,142.1,136.1,136.0,133.3,133.0,132.5,129.1$, $128.8,128.65,128.64,128.34,128.27,128.23,128.19,127.8,127.5,127.4,127.3$, $127.1,126.85,126.81,122.7,122.6,122.3,119.4,119.1,114.4,114.2,109.1,108.7$, 72.0, 71.1, 49.9, 49.2, 48.19, 48.16, 43.6, 43.5, 34.9, 32.8, 28.0, 27.9, 24.9, 24.0, 22.5, 22.2, 19.3, 19.1; ESI-HRMS: $m / z$ Calcd for $\mathrm{C}_{35} \mathrm{H}_{35} \mathrm{~N}_{3} \mathrm{O}+\mathrm{H}^{+}$: 514.2853, found 514.2844 .

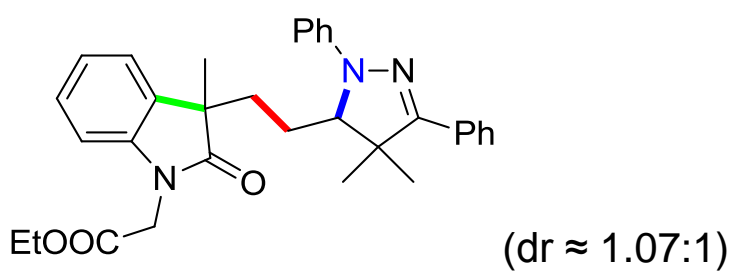

Ethyl 2-(3-(2-(4, 4-dimethyl-1, 3-diphenyl-4, 5-dihydro-1H-pyrazol-5-yl) ethyl)-3methyl-2-oxoindolin-1-yl) acetate (3c)

Yellow oil; (211 mg, 83\% yield); $\mathrm{R}_{f}=0.45$ (hexanes/acetone 5:1, twice); ${ }^{1} \mathrm{H}$ NMR 
$\left(400 \mathrm{MHz}, \mathrm{CDCl}_{3}\right): \delta 7.74(\mathrm{~d}, J=7.2 \mathrm{~Hz}, 2 \mathrm{H}), 7.67(\mathrm{dd}, J=8.0 \mathrm{~Hz}, J=1.6 \mathrm{~Hz}, 2 \mathrm{H})$, 7.41-7.32 (m, 7H), 7.23-7.14 (m, 7H), 7.03 (d, $J=8.0 \mathrm{~Hz}, 2 \mathrm{H}), 6.92(\mathrm{~d}, J=8.0 \mathrm{~Hz}$, 2H), 6.86-6.78 (m, 3H), $6.66(\mathrm{~d}, J=8.0 \mathrm{~Hz}, 1 \mathrm{H}), 6.63(\mathrm{~d}, J=8.0 \mathrm{~Hz}, 1 \mathrm{H}), 6.43(\mathrm{~d}, J$ $=7.2 \mathrm{~Hz}, 1 \mathrm{H}), 4.56-4.48(\mathrm{~m}, 2 \mathrm{H}), 4.31-4.24(\mathrm{~m}, 2 \mathrm{H}), 4.22-4.07(\mathrm{~m}, 4 \mathrm{H}), 3.86(\mathrm{~d}, J=$ $4.8 \mathrm{~Hz}, 1 \mathrm{H}), 3.62(\mathrm{dd}, J=8.0 \mathrm{~Hz}, J=2.8 \mathrm{~Hz}, 1 \mathrm{H}), 2.06(\mathrm{td}, J=12.0 \mathrm{~Hz}, J=5.2 \mathrm{~Hz}$, 1H), 1.88-1.79 (m, 2H), 1.68-1.47 (m, 3H), $1.42(\mathrm{~s}, 3 \mathrm{H}), 1.40(\mathrm{~s}, 3 \mathrm{H}), 1.37(\mathrm{~s}, 3 \mathrm{H})$, $1.35(\mathrm{~s}, 3 \mathrm{H}), 1.33(\mathrm{~s}, 3 \mathrm{H}), 1.30-1.21(\mathrm{~m}, 8 \mathrm{H}), 1.22(\mathrm{t}, J=7.2 \mathrm{~Hz}, 3 \mathrm{H}) ;{ }^{13} \mathrm{C}$ NMR $(100$ $\left.\mathrm{MHz}, \mathrm{CDCl}_{3}\right): \delta 180.4,180.0,167.6,167.5,155.5,155.2,144.8,144.5,142.0,141.8$, $133.1,132.8,132.6,129.0,128.8,128.4,128.3,128.22,128.16,128.0,127.4,126.9$, $123.1,122.9,122.54,122.47,119.4,119.0,114.4,114.1,108.0,107.5,72.1,71.2,61.6$, 50.0, 49.1, 48.22, 48.21, 41.2, 41.1, 35.0, 32.6, 31.9, 29.7, 28.0, 24.7, 24.0, 22.7, 22.4, 22.0, 19.4, 19.0, 14.1; ESI-HRMS: $m / z$ Calcd for $\mathrm{C}_{32} \mathrm{H}_{35} \mathrm{~N}_{3} \mathrm{O}_{3}+\mathrm{H}^{+}$: 510.2751, found 510.2743 .

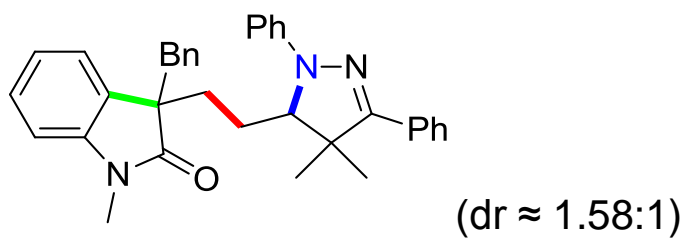

3-benzyl-3-(2-(4, 4-dimethyl-1, 3-diphenyl-4, 5-dihydro-1H-pyrazol-5-yl) ethyl)-1methylindolin-2-one (3f)

Yellow oil; (167 mg, 65\% yield); $\mathrm{R}_{f}=0.25$ (hexanes/acetone 5:1); ${ }^{1} \mathrm{H}$ NMR (400 $\left.\mathrm{MHz}, \mathrm{CDCl}_{3}\right): \delta 7.77(\mathrm{~d}, J=8.8 \mathrm{~Hz}, 2 \mathrm{H}), 7.67(\mathrm{dd}, J=8.0 \mathrm{~Hz}, J=1.6 \mathrm{~Hz}, 2 \mathrm{H})$, 7.43-7.31 (m, 7H), 7.21-7.12 (m, 4H), 7.10-6.98 (m, 9H), $6.91(\mathrm{~d}, J=8.0 \mathrm{~Hz}, 2 \mathrm{H})$, 6.86-6.73 (m, 6H), $6.67(\mathrm{~d}, J=6.8 \mathrm{~Hz}, 3 \mathrm{H}), 6.53-6.48(\mathrm{~m}, 2 \mathrm{H}), 6.21(\mathrm{~d}, J=6.8 \mathrm{~Hz}$, $1 \mathrm{H}), 3.86(\mathrm{~d}, J=8.0 \mathrm{~Hz}, 1 \mathrm{H}), 3.63(\mathrm{dd}, J=8.0 \mathrm{~Hz}, J=3.2 \mathrm{~Hz}, 1 \mathrm{H}), 3.05-2.79(\mathrm{~m}$, $10 \mathrm{H}), 2.21(\mathrm{td}, J=12.0 \mathrm{~Hz}, J=4.0 \mathrm{~Hz}, 1 \mathrm{H}), 2.01-1.87(\mathrm{~m}, 2 \mathrm{H}), 1.72-1.55(\mathrm{~m}, 3 \mathrm{H})$, $1.47(\mathrm{~s}, 3 \mathrm{H}), 1.42(\mathrm{~s}, 3 \mathrm{H}), 1.40(\mathrm{~s}, 3 \mathrm{H}), 1.36(\mathrm{~s}, 3 \mathrm{H}), 1.26-1.18(\mathrm{~m}, 2 \mathrm{H}) ;{ }^{13} \mathrm{C} \mathrm{NMR}$ $\left(100 \mathrm{MHz} \mathrm{CDCl}_{3}\right): \delta 178.9,178.5,155.5,155.1,144.7,144.5,143.9,143.6,135.6$, $135.5,132.53,132.51,130.4,130.2,129.9,129.8,129.0,128.8,128.4,128.3,128.2$, $128.0,127.44,127.36,127.2,126.85,126.82,126.4,126.3,123.3,123.2,122.2,122.0$, 119.4, 119.1, 114.4, 114.3, 107.8, 107.2, 72.0, 71.4, 54.5, 54.4, 50.0, 49.0, 44.8, 44.3, 33.5, 31.0, 28.0, 27.9, 25.73, 25.69, 22.3, 21.9, 19.4, 19.1; ESI-HRMS: $m / z$ Calcd for 
$\mathrm{C}_{35} \mathrm{H}_{35} \mathrm{~N}_{3} \mathrm{O}+\mathrm{H}^{+}:$514.2853, found 514.2846.

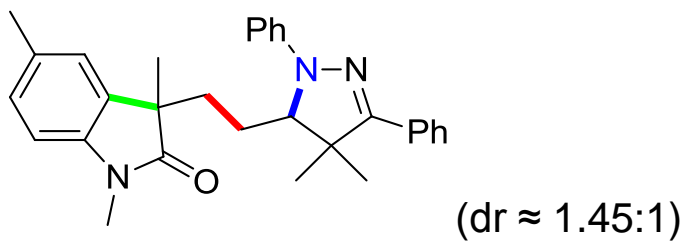

3-(2-(4, 4-dimethyl-1, 3-diphenyl-4, 5-dihydro-1H-pyrazol-5-yl) ethyl)-1, 3, 5trimethylindolin-2-one (3g)

Yellow oil; (194 mg, 86\% yield); $\mathrm{R}_{f}=0.35$ (hexanes/acetone 5:1, twice); ${ }^{1} \mathrm{H}$ NMR $\left(400 \mathrm{MHz}, \mathrm{CDCl}_{3}\right): \delta 7.73(\mathrm{~d}, J=6.8 \mathrm{~Hz}, 2 \mathrm{H}), 7.66(\mathrm{t}, J=8.0 \mathrm{~Hz}, J=1.6 \mathrm{~Hz}, 2 \mathrm{H})$, 7.41-7.30 (m, 6H), 7.24-7.15 (m, 4H), 7.05-6.96 (m, 5H), $6.90(\mathrm{~d}, J=8.4 \mathrm{~Hz}, 2 \mathrm{H})$, 6.86-6.78 (m, 2H), 6.65 (dd, $J=8.0 \mathrm{~Hz}, J=5.2 \mathrm{~Hz}, 2 \mathrm{H}), 6.30$ (s, 1H), 3.83 (d, $J=7.2$ Hz, 1H), 3.59 (dd, $J=8.4 \mathrm{~Hz}, J=2.8 \mathrm{~Hz}, 1 \mathrm{H}), 3.13$ (s, 3H), 3.12 (s, 3H), 2.35 (s, 3H), $2.17(\mathrm{~s}, 3 \mathrm{H}), 2.06-2.00(\mathrm{~m}, 1 \mathrm{H}), 1.87-1.74(\mathrm{~m}, 2 \mathrm{H}), 1.60-1.49(\mathrm{~m}, 2 \mathrm{H}), 1.43(\mathrm{~m}, 3 \mathrm{H})$, $1.41(\mathrm{~s}, 3 \mathrm{H}), 1.37(\mathrm{~s}, 3 \mathrm{H}), 1.34(\mathrm{~s}, 3 \mathrm{H}), 1.31(\mathrm{~s}, 3 \mathrm{H}), 1.29-1.23(\mathrm{~m}, 3 \mathrm{H}), 1.18(\mathrm{~s}, 3 \mathrm{H})$; ${ }^{13} \mathrm{C} \mathrm{NMR}\left(100 \mathrm{MHz}, \mathrm{CDCl}_{3}\right): \delta 180.2,179.9,155.5,155.1,144.7,144.3,140.9,140.7$, $133.3,133.1,132.49,132.46,132.0,131.9,128.8,128.7,128.3,128.23,128.18$, $128.15,128.1,127.7,126.8,123.1,123.0,119.4,119.1,114.4,114.1,107.8,107.3$, 72.1, 71.1, 50.0, 49.0, 48.3, 48.2, 35.0, 32.5, 28.0, 26.1, 26.0, 24.6, 23.9, 22.2, 22.1, 21.1, 19.4, 19.0; ESI-HRMS: $m / z$ Calcd for $\mathrm{C}_{30} \mathrm{H}_{33} \mathrm{~N}_{3} \mathrm{O}+\mathrm{H}^{+}$: 452.2696, found 452.2687 .

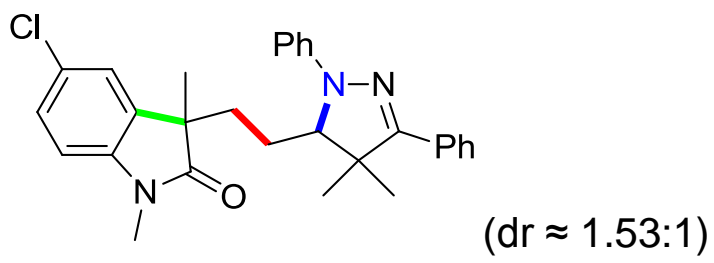

5-chloro-3-(2-(4, 4-dimethyl-1, 3-diphenyl-4, 5-dihydro-1H-pyrazol-5-yl) ethyl)-1, 3dimethylindolin-2-one (3h)

Yellow oil; (191 mg, 81\% yield); $\mathrm{R}_{f}=0.35$ (hexanes/acetone 5:1, twice); ${ }^{1} \mathrm{H}$ NMR $\left(400 \mathrm{MHz}, \mathrm{CDCl}_{3}\right): \delta 7.76(\mathrm{dd}, J=8.0 \mathrm{~Hz}, J=4.2 \mathrm{~Hz}, 2 \mathrm{H}), 7.70(\mathrm{dd}, J=8.0 \mathrm{~Hz}, J=$ $1.2 \mathrm{~Hz}, 2 \mathrm{H}), 7.44-7.18(\mathrm{~m}, 14 \mathrm{H}), 7.08(\mathrm{~d}, J=7.6 \mathrm{~Hz}, 2 \mathrm{H}), 6.95-6.91(\mathrm{~m}, 2 \mathrm{H}), 6.85(\mathrm{t}$, $J=7.2 \mathrm{~Hz}, 1 \mathrm{H}), 6.70(\mathrm{~d}, J=8.4 \mathrm{~Hz}, 2 \mathrm{H}), 6.48(\mathrm{~d}, J=2.4 \mathrm{~Hz}, 1 \mathrm{H}), 3.87(\mathrm{~d}, J=4.2 \mathrm{~Hz}$, 1H), $3.64(\mathrm{dd}, J=8.4 \mathrm{~Hz}, J=2.4 \mathrm{~Hz}, 1 \mathrm{H}), 3.16(\mathrm{~s}, 3 \mathrm{H}), 3.15(\mathrm{~s}, 3 \mathrm{H}), 2.07$ (td, $J=$ $13.2 \mathrm{~Hz}, J=4.2 \mathrm{~Hz}, 1 \mathrm{H}), 1.87(\mathrm{td}, J=13.2 \mathrm{~Hz}, J=4.0 \mathrm{~Hz}, 1 \mathrm{H}), 1.75(\mathrm{td}, J=13.2 \mathrm{~Hz}$, 
$J=3.2 \mathrm{~Hz}, 1 \mathrm{H}), 1.64-1.53(\mathrm{~m}, 3 \mathrm{H}), 1.45(\mathrm{~s}, 3 \mathrm{H}), 1.446(\mathrm{~s}, 3 \mathrm{H}), 1.41(\mathrm{~s}, 3 \mathrm{H}), 1.39(\mathrm{~s}$, $3 \mathrm{H}), 1.34(\mathrm{~s}, 3 \mathrm{H}), 1.39-1.28(\mathrm{~m}, 2 \mathrm{H}), 1.22(\mathrm{~s}, 3 \mathrm{H}) ;{ }^{13} \mathrm{C} \mathrm{NMR}\left(100 \mathrm{MHz}, \mathrm{CDCl}_{3}\right): \delta$ 179.8, 179.4, 155.6, 155.2, 144.6, 144.3, 141.9, 141.7, 135.1, 134.9, 132.5, 132.4, 129.0, 128.9, 128.4, 128.31, 128.29, 128.1, 128.0, 127.9, 127.7, 126.9, 122.9, 122.8, 119.8, 119.7, 114.5, 114.1, 109.0, 108.6, 72.2, 71.1, 50.0, 49.2, 48.6, 48.5, 35.0, 32.6, 28.0, 27.9, 26.22, 26.19, 24.5, 23.7, 22.1, 19.5, 19.1; ESI-HRMS: $\mathrm{m} / \mathrm{z}$ Calcd for $\mathrm{C}_{29} \mathrm{H}_{30} \mathrm{ClN}_{3} \mathrm{O}+\mathrm{H}^{+}: 472.2150$, found 472.2144.

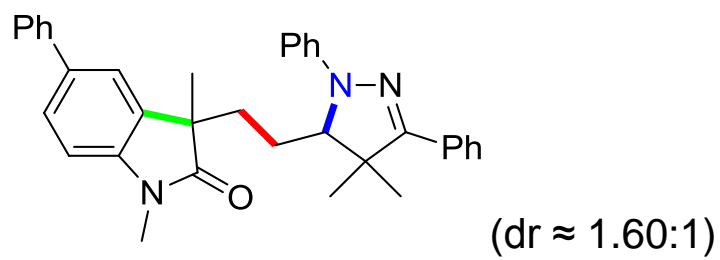

3-(2-(4, 4-dimethyl-1, 3-diphenyl-4, 5-dihydro-1H-pyrazol-5-yl) ethyl)-1, 3-dimethyl5-phenylindolin-2-one (3i)

Yellow oil; (210 mg, 82\% yield); $\mathrm{R}_{f}=0.50$ (hexanes/acetone 5:1, twice); ${ }^{1} \mathrm{H}$ NMR $\left(400 \mathrm{MHz}, \mathrm{CDCl}_{3}\right): \delta 7.23(\mathrm{dd}, J=8.0 \mathrm{~Hz}, J=1.2 \mathrm{~Hz}, 2 \mathrm{H}), 7.67(\mathrm{dd}, J=7.6 \mathrm{~Hz}, J=$ $1.2 \mathrm{~Hz}, 1 \mathrm{H}), 7.53(\mathrm{~d}, J=7.6 \mathrm{~Hz}, 2 \mathrm{H}), 7.47-7.32(\mathrm{~m}, 18 \mathrm{H}), 7.10-7.00(\mathrm{~m}, 6 \mathrm{H}), 6.92(\mathrm{~d}$, $J=8.0 \mathrm{~Hz}, 2 \mathrm{H}), 6.83(\mathrm{t}, J=8.0 \mathrm{~Hz}, 3 \mathrm{H}), 6.75(\mathrm{t}, J=7.2 \mathrm{~Hz}, 1 \mathrm{H}), 6.49(\mathrm{t}, J=7.2 \mathrm{~Hz}$, $1 \mathrm{H}), 3.83(\mathrm{~d}, J=5.2 \mathrm{~Hz}, 1 \mathrm{H}), 3.61(\mathrm{dd}, J=8.4 \mathrm{~Hz}, J=2.4 \mathrm{~Hz}, 1 \mathrm{H}), 3.20(\mathrm{~s}, 3 \mathrm{H}), 3.18$ (s, 3H), 2.10-2.05 (m, 1H), 1.92-1.81 (m, 1H), 1.70-1.61 (m, 1H), 1.58-1.50 (m, 1H), $1.45(\mathrm{~s}, 3 \mathrm{H}), 1.42(\mathrm{~s}, 3 \mathrm{H}), 1.37(\mathrm{~s}, 9 \mathrm{H}), 1.34-1.26(\mathrm{~m}, 7 \mathrm{H}) ;{ }^{13} \mathrm{C}$ NMR $(100 \mathrm{MHz}$, $\left.\mathrm{CDCl}_{3}\right): \delta 180.4,180.0,155.7,155.2,144.6,144.1,142.7,142.5,141.0,140.86,136.2$, $136.1,133.9,133.7,132.5,132.4,128.9,128.85,128.78,128.6,128.4,128.3,128.2$, 127.0, 126.9, 126.84, 126.81, 126.5, 121.20, 121.15, 119.5, 119.1, 114.3, 113.8, 108.3, 107.9, 72.1, 71.0, 50.0, 49.1, 48.5, 48.4, 35.0, 32.7, 28.05, 27.95, 26.22, 26.20, 24.6, 24.0, 23.97, 22.2, 19.5, 19.0; ESI-HRMS: $m / z$ Calcd for $\mathrm{C}_{35} \mathrm{H}_{35} \mathrm{~N}_{3} \mathrm{O}+\mathrm{H}^{+}$: 514.2853, found 514.2845.

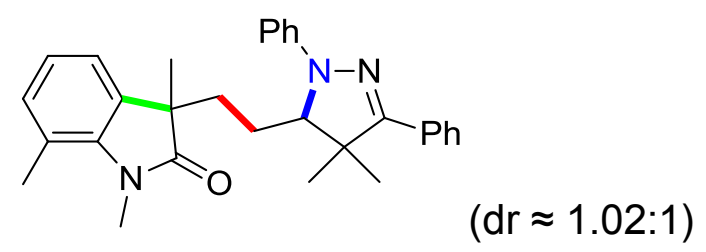

3-(2-(4, 4-dimethyl-1, 3-diphenyl-4, 5-dihydro-1H-pyrazol-5-yl) ethyl)-1, 3, 7trimethylindolin-2-one (3j) 
Yellow oil; (142 mg, 63\% yield); $\mathrm{R}_{f}=0.25$ (hexanes/acetone 5:1, twice); ${ }^{1} \mathrm{H}$ NMR $\left(400 \mathrm{MHz}, \mathrm{CDCl}_{3}\right): \delta 7.72(\mathrm{dd}, J=8.4 \mathrm{~Hz}, J=1.6 \mathrm{~Hz}, 2 \mathrm{H}), 7.67(\mathrm{dd}, J=8.4 \mathrm{~Hz}, J=$ $1.2 \mathrm{~Hz}, 2 \mathrm{H}), 7.38-7.32(\mathrm{~m}, 6 \mathrm{H}), 7.25-7.15(\mathrm{~m}, 5 \mathrm{H}), 7.04-6.78(\mathrm{~m}, 9 \mathrm{H}), 6.69(\mathrm{t}, J=$ $7.6 \mathrm{~Hz}, 1 \mathrm{H}), 6.31(\mathrm{~d}, J=6.8 \mathrm{~Hz}, 1 \mathrm{H}), 3.81(\mathrm{dd}, J=6.8 \mathrm{~Hz}, J=1.6 \mathrm{~Hz}, 1 \mathrm{H}), 3.59$ (dd, $J=8.4 \mathrm{~Hz}, J=2.4 \mathrm{~Hz}, 1 \mathrm{H}), 3.42(\mathrm{~s}, 3 \mathrm{H}), 3.417(\mathrm{~s}, 3 \mathrm{H}), 2.53(\mathrm{~s}, 3 \mathrm{H}), 2.52(\mathrm{~s}, 3 \mathrm{H})$, $2.03(\mathrm{td}, J=12.8 \mathrm{~Hz}, J=4.2 \mathrm{~Hz}, 1 \mathrm{H}), 1.85(\mathrm{td}, J=13.2 \mathrm{~Hz}, J=4.4 \mathrm{~Hz}, 1 \mathrm{H})$, 1.76-1.54 (m, 3H), 1.42 (s, 3H), 1.41 (s, 3H), 1.38 (s, 3H), $1.35(\mathrm{~s}, 3 \mathrm{H}), 1.29$ (s, 3H), 1.27-1.20 (m, 3H), $1.18(\mathrm{~s}, 3 \mathrm{H}) ;{ }^{13} \mathrm{C}$ NMR (100 MHz, $\left.\mathrm{CDCl}_{3}\right): \delta 181.0,181.7,155.6$, $155.2,144.7,144.5,141.1,140.8,134.0,133.7,132.5,131.7,131.2$, 129.0, 128.8, $128.4,128.3,128.22,128.19,126.9,122.6,122.4,120.2,120.1,119.7,119.4,119.2$, 119.1, 114.4, 114.2, 72.1, 71.2, 50.0, 49.2, 47.6, 47.5, 35.2, 33.0, 29.4, 28.04, 28.02, 25.0, 24.3, 22.2, 22.1, 19.5, 19.2, 19.0; ESI-HRMS: $m / z$ Calcd for $\mathrm{C}_{30} \mathrm{H}_{33} \mathrm{~N}_{3} \mathrm{O}+\mathrm{H}^{+}$: 452.2696, found 452.2689.
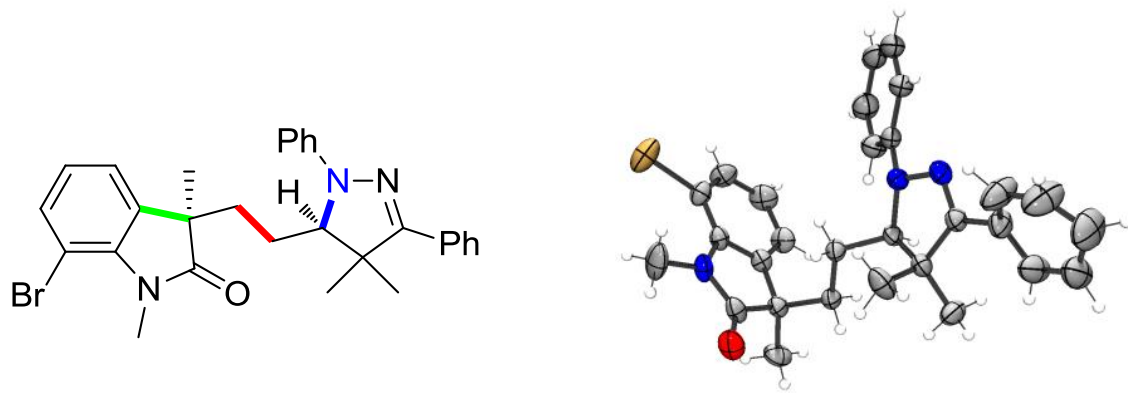

\section{$\mathrm{X}$-ray structure of $3 \mathbf{k}$}

$\left(R^{*}\right)-7-b r o m o-3-\left(2-\left(\left(R^{*}\right)-4,4-d i m e t h y l-1,3-d i p h e n y l-4\right.\right.$, 5-dihydro-1H-pyrazol-5-yl) ethyl)-1, 3-dimethylindolin-2-one (3k)

Colorless solid, mp $134-135{ }^{\circ} \mathrm{C}$; (121 mg, 47\% yield); $\mathrm{R}_{f}=0.35$ (hexanes/acetone 5:1, twice); ${ }^{1} \mathrm{H}$ NMR (400 MHz, $\left.\mathrm{CDCl}_{3}\right): \delta 7.73(\mathrm{dd}, J=8.0 \mathrm{~Hz}, J=1.2 \mathrm{~Hz}, 2 \mathrm{H})$, 7.42-7.33 (m, 3H), 7.24-7.18 (m, 3H), $7.02(\mathrm{dd}, J=8.8 \mathrm{~Hz}, J=0.8 \mathrm{~Hz}, 2 \mathrm{H}), 6.84(\mathrm{t}$, $J=7.2 \mathrm{~Hz}, 1 \mathrm{H}), 6.60(\mathrm{t}, J=7.2 \mathrm{~Hz}, 1 \mathrm{H}), 6.25(\mathrm{dd}, J=7.2 \mathrm{~Hz}, J=1.2 \mathrm{~Hz}, 1 \mathrm{H}), 3.86$ $(\mathrm{d}, J=7.2 \mathrm{~Hz}, 1 \mathrm{H}), 3.53(\mathrm{~s}, 3 \mathrm{H}), 1.84(\mathrm{td}, J=13.2 \mathrm{~Hz}, J=4.4 \mathrm{~Hz}, 1 \mathrm{H}), 1.51(\mathrm{td}, J=$ $13.2 \mathrm{~Hz}, J=3.2 \mathrm{~Hz}, 1 \mathrm{H}), 1.43(\mathrm{~s}, 3 \mathrm{H}), 1.38(\mathrm{~s}, 3 \mathrm{H}), 1.36-1.21(\mathrm{~m}, 2 \mathrm{H}), 1.20(\mathrm{~s}, 3 \mathrm{H})$; ${ }^{13} \mathrm{C} \mathrm{NMR}\left(100 \mathrm{MHz}, \mathrm{CDCl}_{3}\right): \delta 180.6,155.2,144.4,140.3,136.2,133.0,132.4,129.1$, 128.4, 128.3, 126.8, 123.9, 121.2, 119.0, 114.1, 101.9, 71.0, 48.9, 48.0, 32.6, 29.6, 27.9, 25.0, 21.9, 19.0; ESI-HRMS: $m / z$ Calcd for $\mathrm{C}_{29} \mathrm{H}_{30} \mathrm{BrN}_{3} \mathrm{O}+\mathrm{H}^{+}$: 516.1645, found 
516.1649.

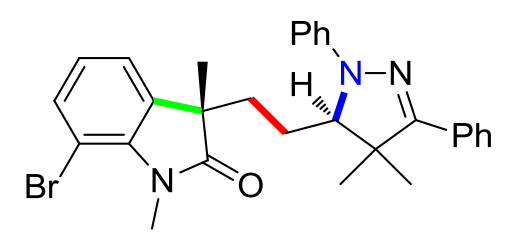

( $\left.S^{*}\right)-7-b r o m o-3-\left(2-\left(\left(R^{*}\right)-4\right.\right.$, 4-dimethyl-1, 3-diphenyl-4, 5-dihydro-1H-pyrazol-5-yl) ethyl)-1, 3-dimethylindolin-2-one (3k')

Yellow oil; (98 mg, 38\% yield); $\mathrm{R}_{f}=0.45$ (hexanes/acetone 3:1, twice); ${ }^{1} \mathrm{H}$ NMR (400 $\left.\mathrm{MHz}, \mathrm{CDCl}_{3}\right): \delta 7.67(\mathrm{~d}, J=8.0 \mathrm{~Hz}, 2 \mathrm{H}), 7.37-7.30(\mathrm{~m}, 4 \mathrm{H}), 7.20(\mathrm{t}, J=7.6 \mathrm{~Hz}, 2 \mathrm{H})$, $7.04(\mathrm{~d}, J=7.2 \mathrm{~Hz}, 1 \mathrm{H}), 6.93(\mathrm{~d}, J=7.6 \mathrm{~Hz}, 2 \mathrm{H}), 6.88(\mathrm{t}, J=8.0 \mathrm{~Hz}, 1 \mathrm{H}), 6.82(\mathrm{t}, J=$ $7.6 \mathrm{~Hz}, 1 \mathrm{H}), 3.63(\mathrm{dd}, J=8.0 \mathrm{~Hz}, J=2.8 \mathrm{~Hz}, 1 \mathrm{H}), 3.52(\mathrm{~s}, 3 \mathrm{H}), 2.00(\mathrm{td}, J=13.2 \mathrm{~Hz}$, $J=4.8 \mathrm{~Hz}, 1 \mathrm{H}), 1.71(\mathrm{td}, J=12.8 \mathrm{~Hz}, J=3.6 \mathrm{~Hz}, 1 \mathrm{H}), 1.58-1.48(\mathrm{~m}, 1 \mathrm{H}), 1.40(\mathrm{~s}$, $3 \mathrm{H}), 1.33(\mathrm{~s}, 3 \mathrm{H}), 1.28(\mathrm{~s}, 3 \mathrm{H}), 1.26-1.23(\mathrm{~m}, 1 \mathrm{H}) ;{ }^{13} \mathrm{C} \mathrm{NMR}\left(100 \mathrm{MHz}, \mathrm{CDCl}_{3}\right): \delta$ $180.3,155.5,144.7,140.6,136.6,133.6,132.5,128.9$, 128.3, 128.2, 126.9, 123.6, $121.3,119.6,114.4,102.6,71.9,49.9,47.9,35.0,29.6,28.0,24.1,22.2,19.4$; ESI-HRMS: $m / z$ Calcd for $\mathrm{C}_{29} \mathrm{H}_{30} \mathrm{BrN}_{3} \mathrm{O}+\mathrm{H}^{+}:$516.1645, found 516.1640.

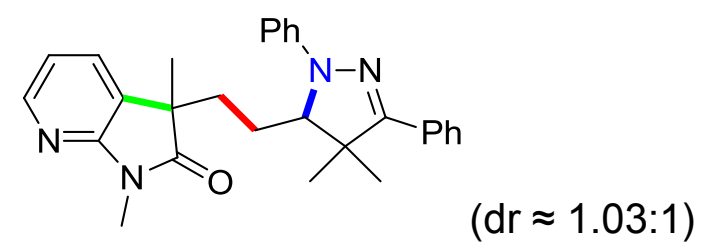

3-(2-(4, 4-dimethyl-1, 3-diphenyl-4, 5-dihydro-1H-pyrazol-5-yl) ethyl)-1, 3-dimethyl1H-pyrrolo [2, 3-b] pyridin-2(3H)-one (3l)

Yellow oil; (123 mg, 56\% yield); $\mathrm{R}_{f}=0.40$ (hexanes/ethyl acetate 5:1); ${ }^{1} \mathrm{H}$ NMR (400 $\left.\mathrm{MHz}, \mathrm{CDCl}_{3}\right): \delta 8.13(\mathrm{dd}, J=5.2 \mathrm{~Hz}, J=1.2 \mathrm{~Hz}, 2 \mathrm{H}), 7.60(\mathrm{dd}, J=5.2 \mathrm{~Hz}, J=1.2$ $\mathrm{Hz}, 2 \mathrm{H}), 7.74(\mathrm{~d}, J=7.2 \mathrm{~Hz}, 2 \mathrm{H}), 7.66(\mathrm{~d}, J=6.8 \mathrm{~Hz}, 2 \mathrm{H}), 7.41-7.31(\mathrm{~m}, 7 \mathrm{H})$, 7.24-6.99 (m, 4H), 6.97-6.89 (m, 2H), 6.87-6.81 (m, 3H), $6.66(\mathrm{dd}, J=7.2 \mathrm{~Hz}, J=$ $5.6 \mathrm{~Hz}, 1 \mathrm{H}), 6.50(\mathrm{dd}, J=7.2 \mathrm{~Hz}, J=5.2 \mathrm{~Hz}, 1 \mathrm{H}), 3.88(\mathrm{~d}, J=5.2 \mathrm{~Hz}, 1 \mathrm{H}), 3.69(\mathrm{dd}$, $J=7.2 \mathrm{~Hz}, J=2.8 \mathrm{~Hz}, 1 \mathrm{H}), 3.23(\mathrm{~s}, 6 \mathrm{H}), 1.97-1.93(\mathrm{~m}, 1 \mathrm{H}), 1.86-1.81(\mathrm{~m}, 1 \mathrm{H})$, 1.76-1.72 (m, 1H), 1.66-1.50 (m, 2H), 1.49 (s, 3H), 1.45 (s, 3H), 1.40 (s, 3H), 1.37 (s, $3 \mathrm{H}), 1.33(\mathrm{~s}, 3 \mathrm{H}), 1.30-1.23(\mathrm{~m}, 3 \mathrm{H}), 1.19(\mathrm{~s}, 3 \mathrm{H}) ;{ }^{13} \mathrm{C}$ NMR $\left(100 \mathrm{MHz}, \mathrm{CDCl}_{3}\right): \delta$ $179.9,179.6,156.8,156.7,155.6,155.3,146.8,146.3,144.7,144.4,132.4,129.7$, $129.6,129.1,128.9,128.41,128.37,128.32$, 128.29, 127.8, 127.4, 126.85, 126.83, 
119.6, 119.1, 118.3, 118.0, 114.4, 114.2, 71.8, 71.0, 49.9, 49.0, 48.0, 47.8, 34.2, 32.0, 28.0, 27.9, 25.25, 25.18, 24.1, 23.0, 22.4, 21.9, 19.3, 19.0; ESI-HRMS: $m / z$ Calcd for $\mathrm{C}_{28} \mathrm{H}_{30} \mathrm{~N}_{4} \mathrm{O}+\mathrm{H}^{+}:$439.2492, found 439.2489.

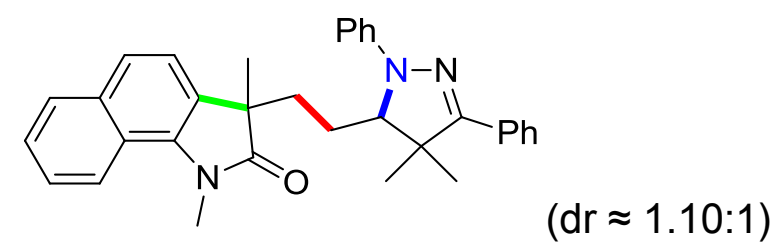

3-(2-(4, 4-dimethyl-1, 3-diphenyl-4, 5-dihydro-1H-pyrazol-5-yl) ethyl)-1, 3-dimethyl1H-benzo[g]indol-2(3H)-one $(3 \mathrm{~m})$

Yellow oil; (185 mg, 76\% yield); $\mathrm{R}_{f}=0.40$ (hexanes/ethyl acetate 5:1); ${ }^{1} \mathrm{H}$ NMR (400 $\left.\mathrm{MHz} \mathrm{CDCl}_{3}\right): \delta 7.74(\mathrm{~d}, J=6.8 \mathrm{~Hz}, 2 \mathrm{H}), 7.70(\mathrm{~d}, J=7.6 \mathrm{~Hz}, 1 \mathrm{H}), 7.65(\mathrm{~d}, J=1.6 \mathrm{~Hz}$, 1H), 7.63-7.53 (m, 2H), 7.47-7.30 (m, 13H), 7.24-7.02 (m, 6H), 6.88-6.84 (m, 3H), $6.80(\mathrm{~d}, J=8.0 \mathrm{~Hz}, 2 \mathrm{H}), 6.73(\mathrm{t}, J=7.2 \mathrm{~Hz}, 1 \mathrm{H}), 6.65(\mathrm{~d}, J=7.2 \mathrm{~Hz}, 1 \mathrm{H}), 3.87$ (d, $J=$ $5.2 \mathrm{~Hz}, 1 \mathrm{H}), 3.57(\mathrm{dd}, J=8.4 \mathrm{~Hz}, J=2.8 \mathrm{~Hz}, 1 \mathrm{H}), 3.46(\mathrm{~s}, 3 \mathrm{H}), 3.44(\mathrm{~s}, 3 \mathrm{H}), 2.54(\mathrm{td}$, $J=12.0 \mathrm{~Hz}, J=4.0 \mathrm{~Hz}, 1 \mathrm{H}), 2.38(\mathrm{td}, J=12.0 \mathrm{~Hz}, J=4.0 \mathrm{~Hz}, 1 \mathrm{H}), 1.91(\mathrm{td}, J=16.0$ $\mathrm{Hz}, J=3.2 \mathrm{~Hz}, 1 \mathrm{H}), 1.76(\mathrm{td}, J=12.8 \mathrm{~Hz}, J=4.0 \mathrm{~Hz}, 1 \mathrm{H}), 1.69-1.53(\mathrm{~m}, 2 \mathrm{H}), 1.47$ (s, $3 \mathrm{H}), 1.44(\mathrm{~s}, 3 \mathrm{H}), 1.40(\mathrm{~s}, 3 \mathrm{H}), 1.37$ (s, 3H), $1.32(\mathrm{~s}, 6 \mathrm{H}), 1.26(\mathrm{~s}, 2 \mathrm{H}) ;{ }^{13} \mathrm{C}$ NMR (100 $\left.\mathrm{MHz}, \mathrm{CDCl}_{3}\right): \delta 173.2,172.8,155.5,155.3,144.5,144.4,137.5,136.9,136.5,133.2$, 133.1, 132.6, 132.5, 130.4, 129.0, 128.7, 128.54, 128.51, 128.3, 128.22, 128.17, 128.1, 127.3, 126.9, 126.8, 126.3, 126.1, 126.0, 125.4, 122.44, 125.40, 122.36, 122.3, 119.7, 119.5, 119.2, 118.9, 114.3, 114.0, 108.3, 108.0, 72.0, 71.2, 50.0, 49.1, 47.51, 47.47, 40.7, 36.5, 32.9, 31.2, 29.53, 29.50, 28.0, 27.9, 23.22, 23.17, 19.4, 18.9; ESI-HRMS: $m / z$ Calcd for $\mathrm{C}_{33} \mathrm{H}_{33} \mathrm{~N}_{3} \mathrm{O}+\mathrm{H}^{+}:$488.2696, found 488.2687.

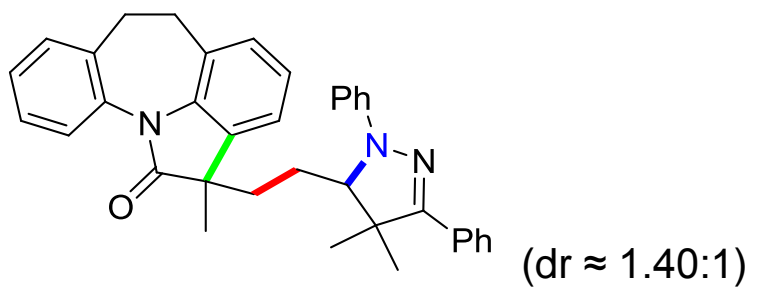

7-(2-(4, 4-dimethyl-1, 3-diphenyl-4, 5-dihydro-1H-pyrazol-5-yl) ethyl)-7-methyl-11, 12-dihydrobenzo [6, 7] azepino [3, 2, 1-hi] indol-6(7H)-one (3n)

Yellow oil; (189 mg, 72\% yield); $\mathrm{R}_{f}=0.50$ (hexanes/acetone 5:1, twice); ${ }^{1} \mathrm{H}$ NMR $\left(400 \mathrm{MHz}, \mathrm{CDCl}_{3}\right): \delta 7.73(\mathrm{~d}, J=7.2 \mathrm{~Hz}, 3 \mathrm{H}), 7.67(\mathrm{~d}, J=7.2 \mathrm{~Hz}, 3 \mathrm{H}), 7.40-7.28$ (m, 7H), 7.25-7.12 (m, 9H), 7.07-7.01 (m, 3H), 7.00-6.98 (m, 2H), 6.95-6.87 (m, 3H), 
6.86-6.79 (m, 2H), $6.75(\mathrm{t}, J=7.4 \mathrm{~Hz}, 1 \mathrm{H}), 6.36(\mathrm{~d}, J=6.0 \mathrm{~Hz}, 1 \mathrm{H}), 3.88(\mathrm{~d}, J=6.0$ $\mathrm{Hz}, 1 \mathrm{H}), 3.66(\mathrm{~d}, J=6.0 \mathrm{~Hz}, 1 \mathrm{H}), 2.97-2.87(\mathrm{~m}, 8 \mathrm{H}), 2.15(\mathrm{td}, J=12.8 \mathrm{~Hz}, J=4.8$ $\mathrm{Hz}, 1 \mathrm{H}), 1.99$ (t, $J=11.2 \mathrm{~Hz}, 1 \mathrm{H}), 1.80$ (t, $J=12.8 \mathrm{~Hz}, 1 \mathrm{H}), 1.70-1.45$ (m, 5H), 1.41 $(\mathrm{s}, 6 \mathrm{H}), 1.38(\mathrm{~s}, 3 \mathrm{H}), 1.36(\mathrm{~s}, 3 \mathrm{H}), 1.32(\mathrm{~s}, 6 \mathrm{H}) ;{ }^{13} \mathrm{C} \mathrm{NMR}\left(100 \mathrm{MHz}, \mathrm{CDCl}_{3}\right): \delta$ 181.0, $180.5,155.4,155.1,144.7,144.4,140.4,140.1,136.62$, 136.59, 136.1, 136.0, 133.6, $133.2,132.5,129.8,129.5,129.3,129.1,128.9,128.4,128.3,128.24,128.19,126.8$, $126.5,126.4,126.3,126.2,125.8,124.7,124.6,122.5,122.4,120.02,119.99,119.4$, $119.1,114.4,114.2,72.0,71.2,49.8,49.2,47.9,33.9,33.8,33.6,33.5,28.0,27.9$, 22.4, 22.1, 19.4, 19.1; ESI-HRMS: $m / z$, Calcd for $\mathrm{C}_{36} \mathrm{H}_{35} \mathrm{~N}_{3} \mathrm{O}+\mathrm{H}^{+}$: 526.2853, found 526.2840 .

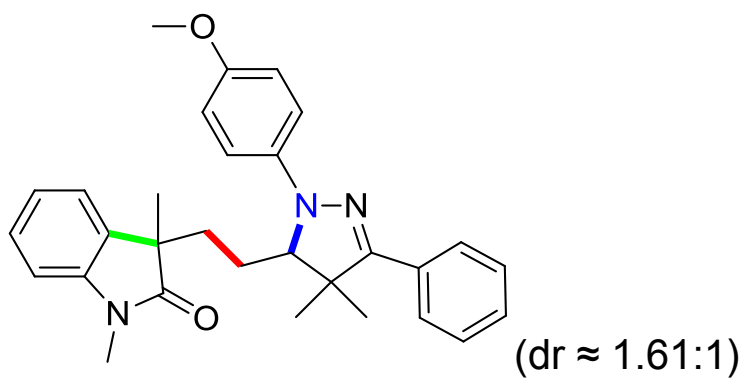

3-(2-(1-(4-methoxyphenyl)-4, 4-dimethyl-3-phenyl-4, 5-dihydro-1H-pyrazol-5-yl) ethyl)-1, 3-dimethylindolin-2-one (3o)

Yellow oil; (198 mg, 85\% yield); $\mathrm{R}_{f}=0.30$ (hexanes/acetone 5:1); ${ }^{1} \mathrm{H}$ NMR (400 $\left.\mathrm{MHz}, \mathrm{CDCl}_{3}\right): \delta 7.73(\mathrm{~d}, J=6.8 \mathrm{~Hz}, 2 \mathrm{H}), 7.68(\mathrm{dd}, J=8.0 \mathrm{~Hz}, J=1.6 \mathrm{~Hz}, 2 \mathrm{H})$, 7.41-7.32 (m, 7H), 7.30-7.16 (m, 2H), $7.10(\mathrm{dd}, J=7.2 \mathrm{~Hz}, J=0.8 \mathrm{~Hz}, 1 \mathrm{H}), 7.00(\mathrm{dd}$, $J=6.8 \mathrm{~Hz}, J=2.4 \mathrm{~Hz}, 2 \mathrm{H}), 6.91-6.78(\mathrm{~m}, 9 \mathrm{H}), 6.57(\mathrm{~d}, J=6.8 \mathrm{~Hz}, 1 \mathrm{H}), 3.85(\mathrm{~s}, 3 \mathrm{H})$, $3.80(\mathrm{~s}, 3 \mathrm{H}), 3.71(\mathrm{dd}, J=8.4 \mathrm{~Hz}, J=2.0 \mathrm{~Hz}, 1 \mathrm{H}), 3.43(\mathrm{~d}, J=8.4 \mathrm{~Hz}, 1 \mathrm{H}), 3.19$ (s, $3 \mathrm{H}), 3.18(\mathrm{~s}, 3 \mathrm{H}), 2.02(\mathrm{td}, J=16.0 \mathrm{~Hz}, J=4.0 \mathrm{~Hz}, 1 \mathrm{H}), 1.88(\mathrm{td}, J=16.0 \mathrm{~Hz}, J=4.0$ Hz, 1H), 1.78-1.47 (m, 4H), 1.44 (s, 3H), 1.41 (s, 3H), 1.40 (s, 3H), 1.34 (s, 3H), 1.31 (s, 3H), 1.29-1.275 (m, 2H), 1.25(s, 3H); ${ }^{13} \mathrm{C}$ NMR (100 MHz, $\left.\mathrm{CDCl}_{3}\right): \delta 180.2$, $180.0,155.6,154.8,154.2,153.6,143.3,143.1,139.5,139.0,133.3,133.1,132.59$, $132.56,128.26,128.18,128.0,127.9,127.4,126.74,126.69,122.5,122.4,122.22$, 122.21, 117.8, 116.4, 114.4, 114.2, 108.0, 107.6, 73.7, 72.2, 55.6, 55.5, 49.9, 49.1, $48.24,48.15,35.0,33.0,27.4,27.2,26.04,255.99,24.3,23.7,22.05,22.00,19.2,19.1$; ESI-HRMS: $m / z$ Calcd for $\mathrm{C}_{30} \mathrm{H}_{33} \mathrm{~N}_{3} \mathrm{O}_{2}+\mathrm{H}^{+}$: 468.2646, found 468.2639. 


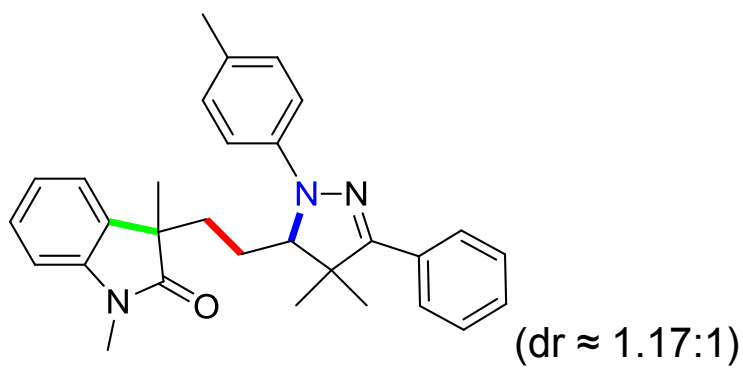

3-(2-(4, 4-dimethyl-3-phenyl-1-(p-tolyl)-4, 5-dihydro-1H-pyrazol-5-yl) ethyl)-1, 3dimethylindolin-2-one (3p)

Yellow oil; (167 mg, 74\% yield); $\mathrm{R}_{f}=0.55$ (hexanes/acetone 5:1); ${ }^{1} \mathrm{H}$ NMR (400 $\left.\mathrm{MHz}, \mathrm{CDCl}_{3}\right): \delta 7.71(\mathrm{~d}, J=6.8 \mathrm{~Hz}, 2 \mathrm{H}), 7.65(\mathrm{~d}, J=6.4 \mathrm{~Hz}, 2 \mathrm{H}), 7.39-7.29(\mathrm{~m}, 6 \mathrm{H})$, 7.24-7.14 (m, 3H), 7.08-6.98 (m, 5H), $6.93(\mathrm{~d}, J=8.4 \mathrm{~Hz}, 2 \mathrm{H}), 6.83-6.74(\mathrm{~m}, 5 \mathrm{H})$, $6.47(\mathrm{~d}, J=6.8 \mathrm{~Hz}, 1 \mathrm{H}), 3.76(\mathrm{dd}, J=6.4 \mathrm{~Hz}, J=1.6 \mathrm{~Hz}, 1 \mathrm{H}), 3.53(\mathrm{dd}, J=8.0 \mathrm{~Hz}, J$ $=3.2 \mathrm{~Hz}, 1 \mathrm{H}), 3.16(\mathrm{~s}, 3 \mathrm{H}), 3.15(\mathrm{~s}, 3 \mathrm{H}), 2.33(\mathrm{~s}, 3 \mathrm{H}), 2.26(\mathrm{~s}, 3 \mathrm{H}), 2.00(\mathrm{td}, J=13.2$ $\mathrm{Hz}, J=5.2 \mathrm{~Hz}, 1 \mathrm{H}), 1.84(\mathrm{td}, J=13.2 \mathrm{~Hz}, J=3.6 \mathrm{~Hz}, 1 \mathrm{H}), 1.75(\mathrm{td}, J=13.2 \mathrm{~Hz}, J=$ $3.6 \mathrm{~Hz}, 1 \mathrm{H}), 1.68-1.51(\mathrm{~m}, 2 \mathrm{H}), 1.40(\mathrm{~s}, 3 \mathrm{H}), 1.396$ (s, 3H), 1.37 (s, 3H), $1.32(\mathrm{~s}, 3 \mathrm{H})$, $1.31(\mathrm{~s}, 3 \mathrm{H}), 1.29-1.23(\mathrm{~m}, 3 \mathrm{H}), 1.21(\mathrm{~s}, 3 \mathrm{H}) ;{ }^{13} \mathrm{C} \mathrm{NMR}\left(100 \mathrm{MHz}, \mathrm{CDCl}_{3}\right): \delta 180.3$, $180.0,155.3,154.7,143.3,143.1,142.8,142.4,133.4,133.1,132.63,132.60,129.5$, $129.4,129.0,128.4,128.3,128.2,128.08,128.06,127.9,127.4,126.81,126.79$, $122.51,122.48,122.34,122.26,115.0,114.5,108.1,107.6,72.5,71.6,49.9,49.1$, $48.3,48.1,34.9,32.8,29.6,27.8,26.1,26.0,24.5,23.7,22.3,22.1,20.54,20.51,19.4$, 19.1; ESI-HRMS: $m / z$, Calcd for $\mathrm{C}_{30} \mathrm{H}_{33} \mathrm{~N}_{3} \mathrm{O}+\mathrm{H}^{+}$: 452.2696, found 452.2690.

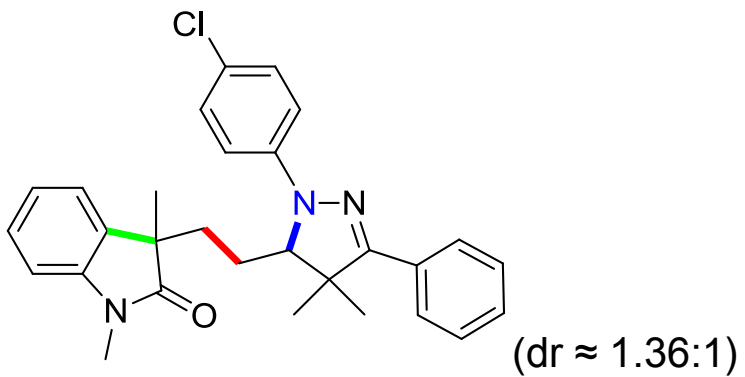

3-(2-(1-(4-chlorophenyl)-4, 4-dimethyl-3-phenyl-4, 5-dihydro-1H-pyrazol-5-yl) ethyl)-1, 3-dimethylindolin-2-one (3q)

Yellow oil; (212 mg, 90\% yield); $\mathrm{R}_{f}=0.30$ (hexanes/acetone 5:1); ${ }^{1} \mathrm{H}$ NMR (400 $\left.\mathrm{MHz}, \mathrm{CDCl}_{3}\right): \delta 7.71(\mathrm{dd}, J=8.0 \mathrm{~Hz}, J=1.6 \mathrm{~Hz}, 2 \mathrm{H}), 7.75(\mathrm{dd}, J=8.0 \mathrm{~Hz}, J=1.6$ $\mathrm{Hz}, 2 \mathrm{H}), 7.41-7.32(\mathrm{~m}, 6 \mathrm{H}), 7.28-7.24(\mathrm{~m}, 2 \mathrm{H}), 7.19(\mathrm{td}, J=7.6 \mathrm{~Hz}, J=1.2 \mathrm{~Hz}, 1 \mathrm{H})$, 7.16-7.07 (m, 6H), $6.93(\mathrm{dd}, J=7.2 \mathrm{~Hz}, J=2.8 \mathrm{~Hz}, 2 \mathrm{H}), 6.87(\mathrm{t}, J=8.2 \mathrm{~Hz}, 1 \mathrm{H})$, 6.80-6.75 (m, 3H), $6.44(\mathrm{~d}, J=7.2 \mathrm{~Hz}, 1 \mathrm{H}), 3.79(\mathrm{~d}, J=4.8 \mathrm{~Hz}, 1 \mathrm{H}), 3.57(\mathrm{dd}, J=$ 
$8.0 \mathrm{~Hz}, J=2.4 \mathrm{~Hz}, 1 \mathrm{H}), 3.15(\mathrm{~s}, 3 \mathrm{H}), 3.147(\mathrm{~s}, 3 \mathrm{H}), 2.04(\mathrm{td}, J=12.0 \mathrm{~Hz}, J=4.0 \mathrm{~Hz}$, $1 \mathrm{H}), 1.86(\mathrm{td}, J=12.0 \mathrm{~Hz}, J=4.0 \mathrm{~Hz}, 1 \mathrm{H}), 1.76(\mathrm{td}, J=12.0 \mathrm{~Hz}, J=4.0 \mathrm{~Hz}, 1 \mathrm{H})$, 1.63-1.50 (m, 3H), 1.42 (s, 3H), 1.39 (s, 3H), 1.37 (s, 3H), 1.33 (s, 3H), 1.31 (s, 3H), 1.29-1.24 (m, 2H), 1.20 (s, 3H); ${ }^{13} \mathrm{C}$ NMR (100 MHz, $\left.\mathrm{CDCl}_{3}\right): \delta 180.1,179.8,156.0$, $155.9,143.3,143.2,143.0,133.2,132.9,132.2,128.9,128.7,128.5,128.4,128.3$, $128.0,127.5,126.9,124.0,123.7,122.7,122.5,122.11,122.07,115.3,115.2,108.1$, 107.6, 72.0, 71.2, 50.1, 49.2, 48.2, 48.1, 34.7, 32.6, 28.0, 27.9, 26.1, 26.0, 24.5, 23.7, 22.1, 21.8, 19.4, 19.0; ESI-HRMS: $m / z$ Calcd for $\mathrm{C}_{29} \mathrm{H}_{30} \mathrm{ClN}_{3} \mathrm{O}+\mathrm{H}^{+}: 472.2150$, found 472.2141 .

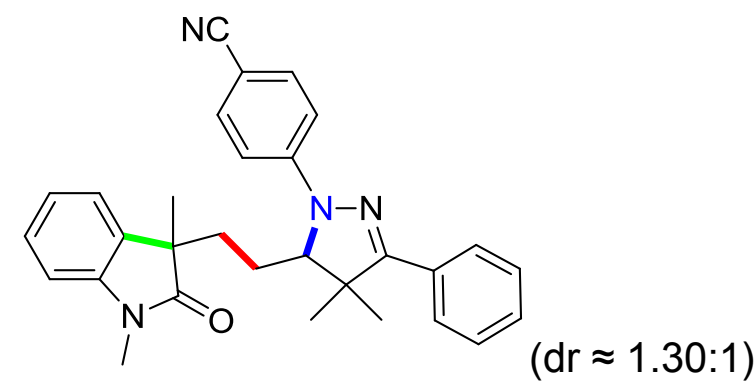

4-(5-(2-(1, 3-dimethyl-2-oxoindolin-3-yl) ethyl)-4, 4-dimethyl-3-phenyl-4, 5dihydro-1H-pyrazol-1-yl) benzonitrile (3r)

Yellow oil; (204 mg, 94\% yield); $\mathrm{R}_{f}=0.30$ (hexanes/acetone 5:1); ${ }^{1} \mathrm{H}$ NMR (400 $\left.\mathrm{MHz} \mathrm{CDCl}_{3}\right): \delta 7.72(\mathrm{dd}, J=8.0 \mathrm{~Hz}, J=4.4 \mathrm{~Hz}, 2 \mathrm{H}), 7.66(\mathrm{dd}, J=8.0 \mathrm{~Hz}, J=4.4$ $\mathrm{Hz}, 2 \mathrm{H}), 7.45-7.37$ (m, 10H), 7.29 (d, J = 7.6 Hz, 1H), 7.23-7.07 (m, 3H), 6.97 (d, $J$ $=8.8 \mathrm{~Hz}, 2 \mathrm{H}), 6.86-6.77(\mathrm{~m}, 5 \mathrm{H}), 6.47(\mathrm{~d}, J=7.2 \mathrm{~Hz}, 1 \mathrm{H}), 3.87(\mathrm{~d}, J=4.8 \mathrm{~Hz}, 1 \mathrm{H})$, $3.69(\mathrm{dd}, J=8.0 \mathrm{~Hz}, J=2.4 \mathrm{~Hz}, 1 \mathrm{H}), 3.16(\mathrm{~s}, 3 \mathrm{H}), 3.14(\mathrm{~s}, 3 \mathrm{H}), 2.01(\mathrm{td}, J=12.0 \mathrm{~Hz}$, $J=4.0 \mathrm{~Hz}, 1 \mathrm{H}), 1.84(\mathrm{td}, J=12.0 \mathrm{~Hz}, J=4.0 \mathrm{~Hz}, 1 \mathrm{H}), 1.71(\mathrm{td}, J=12.0 \mathrm{~Hz}, J=4.0$ $\mathrm{Hz}, 1 \mathrm{H}), 1.61-1.48$ (m, 3H), 1.46 (s, 3H), 1.39 (s, 3H), 1.38 (s, 6H), 1.34 (s, 3H), $1.33-1.25(\mathrm{~m}, 1 \mathrm{H}), 1.21(\mathrm{~s}, 3 \mathrm{H}), 1.19-1.05(\mathrm{~m}, 1 \mathrm{H}) ;{ }^{13} \mathrm{C} \mathrm{NMR}\left(100 \mathrm{MHz}, \mathrm{CDCl}_{3}\right): \delta$ $179.9,179.6,158.4,158.2$, 146.8, 146.6, 143.3, 143.0, 133.3, 133.2, 133.0, 132.6, 131.42, 131.39, 129.1, 129.0, 128.5, 128.4, 128.1, 127.7, 127.05, 127.01, 122.6, 122.5, $122.0,121.8,120.3,113.1,112.7,108.2,107.8,100.0,99.9,71.1,70.5,50.2,49.4$, $48.1,48.0,34.4,32.4,28.4,28.3,26.1,26.0,24.4,23.7,22.2,21.8,19.1,18.8$; ESI-HRMS: $m / z$ Calcd for $\mathrm{C}_{30} \mathrm{H}_{30} \mathrm{~N}_{4} \mathrm{O}+\mathrm{H}^{+}:$463.2492, found 463.2485. 


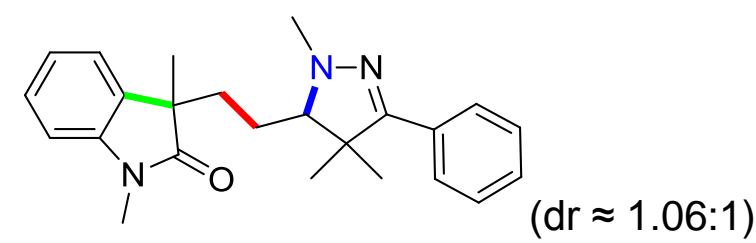

1, 3-dimethyl-3-(2-(1, 4, 4-trimethyl-3-phenyl-4, 5-dihydro-1H-pyrazol-5-yl) ethyl) indolin-2-one (3s)

(Major) Yellow oil; (53 mg, 28\% yield); $\mathrm{R}_{f}=0.25$ (hexanes/acetone 5:1, twice); ${ }^{1} \mathrm{H}$ NMR (400 MHz, $\left.\mathrm{CDCl}_{3}\right): \delta 7.57(\mathrm{dd}, J=8.0 \mathrm{~Hz}, J=1.6 \mathrm{~Hz}, 2 \mathrm{H}), 7.33-7.29(\mathrm{~m}, 4 \mathrm{H})$, $7.20(\mathrm{~d}, J=7.2 \mathrm{~Hz}, 1 \mathrm{H}), 7.09(\mathrm{t}, J=7.2 \mathrm{~Hz}, 1 \mathrm{H}), 6.87$ (d, $J=8.0 \mathrm{~Hz}, 1 \mathrm{H}), 3.24(\mathrm{~s}$, $3 \mathrm{H}), 2.75(\mathrm{~s}, 3 \mathrm{H}), 2.46(\mathrm{dd}, J=8.0 \mathrm{~Hz}, J=4.0 \mathrm{~Hz}, 1 \mathrm{H}), 2.05(\mathrm{td}, J=12.4 \mathrm{~Hz}, J=4.8$ $\mathrm{Hz}, 1 \mathrm{H}), 1.93(\mathrm{td}, J=12.8 \mathrm{~Hz}, J=4.4 \mathrm{~Hz}, 1 \mathrm{H}), 1.40(\mathrm{~s}, 3 \mathrm{H}), 1.31(\mathrm{~s}, 3 \mathrm{H}), 1.26-1.14$ $(\mathrm{m}, 1 \mathrm{H}), 0.99(\mathrm{~s}, 3 \mathrm{H}) ;{ }^{13} \mathrm{C}$ NMR $\left(100 \mathrm{MHz}, \mathrm{CDCl}_{3}\right): \delta$ 180.2, 158.1, 143.4, 133.4, $132.7,128.18,128.16,128.0,126.9,122.6,122.2,108.1,78.1,50.2,48.4,41.8,35.3$, 26.1, 25.7, 23.8, 22.0, 18.4; ESI-HRMS: $m / z$ Calcd for $\mathrm{C}_{24} \mathrm{H}_{29} \mathrm{~N}_{3} \mathrm{O}+\mathrm{H}^{+}$: 376.2383, found 376.2381 .

(Minor) Yellow oil; (50 mg, 27\% yield); $\mathrm{R}_{f}=0.30$ (hexanes/acetone 5:1, twice); ${ }^{1} \mathrm{H}$ NMR (400 MHz, $\left.\mathrm{CDCl}_{3}\right): \delta 7.58(\mathrm{dd}, J=8.4 \mathrm{~Hz}, J=1.4 \mathrm{~Hz}, 2 \mathrm{H}), 7.35-7.27(\mathrm{~m}, 4 \mathrm{H})$, $7.20(\mathrm{~d}, J=6.4 \mathrm{~Hz}, 1 \mathrm{H}), 7.10(\mathrm{td}, J=7.2 \mathrm{~Hz}, 0.8 \mathrm{~Hz}, 1 \mathrm{H}), 6.87(\mathrm{~d}, J=8.0 \mathrm{~Hz}, 1 \mathrm{H})$, $3.24(\mathrm{~s}, 3 \mathrm{H}), 2.69(\mathrm{~s}, 3 \mathrm{H}), 2.42(\mathrm{dd}, J=8.0 \mathrm{~Hz}, J=4.4 \mathrm{~Hz}, 1 \mathrm{H}), 2.13(\mathrm{td}, J=13.2 \mathrm{~Hz}$, $J=4.8 \mathrm{~Hz}, 1 \mathrm{H}), 1.85(\mathrm{td}, J=13.2 \mathrm{~Hz}, J=4.0 \mathrm{~Hz}, 1 \mathrm{H}), 1.40(\mathrm{~s}, 3 \mathrm{H}), 1.34(\mathrm{~s}, 3 \mathrm{H})$, 1.32-1.22 (m, 2H) , 1.07 (s, 3H); ${ }^{13} \mathrm{C}$ NMR (100 MHz, $\left.\mathrm{CDCl}_{3}\right): \delta 180.2,158.4,143.4$, 133.3, 132.7, 128.22, 128.18, 127.9, 127.0, 122.6, 122.3, 108.1, 78.5, 50.3, 48.4, 41.9, 35.5, 26.2, 25.6, 23.9, 22.0, 18.4; ESI-HRMS: $\mathrm{m} / \mathrm{z}$ Calcd for $\mathrm{C}_{24} \mathrm{H}_{29} \mathrm{~N}_{3} \mathrm{O}+\mathrm{H}^{+}$: 376.2383, found 376.2385.

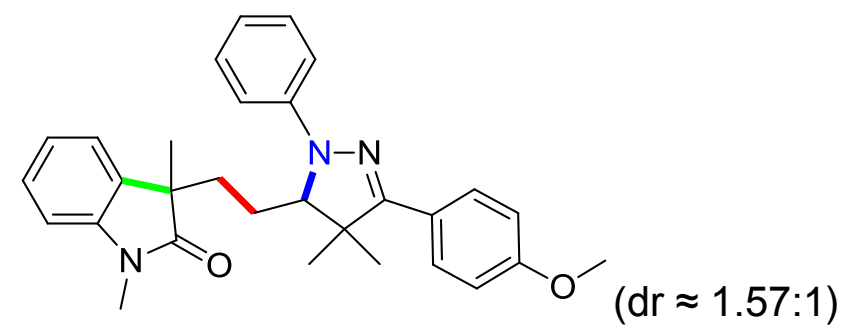

3-(2-(3-(4-methoxyphenyl)-4, 4-dimethyl-1-phenyl-4, 5-dihydro-1H-pyrazol-5-yl) ethyl)-1, 3-dimethylindolin-2-one (3v)

Yellow oil; (184 mg, 79\% yield); $\mathrm{R}_{f}=0.35$ (hexanes/acetone 5:1, twice); ${ }^{1} \mathrm{H}$ NMR $\left(400 \mathrm{MHz}, \mathrm{CDCl}_{3}\right): \delta 7.67(\mathrm{~d}, J=8.8 \mathrm{~Hz}, 2 \mathrm{H}), 7.61(\mathrm{~d}, J=8.4 \mathrm{~Hz}, 2 \mathrm{H}), 7.23-7.15(\mathrm{~m}$, 
6H), 7.08-6.99 (m, 4H), 6.94-6.74 (m, 11H), $6.47(\mathrm{~d}, J=8.8 \mathrm{~Hz}, 1 \mathrm{H}), 3.84(\mathrm{~s}, 3 \mathrm{H})$, $3.82(\mathrm{~s}, 3 \mathrm{H}), 3.78(\mathrm{dd}, J=9.2 \mathrm{~Hz}, J=5.2 \mathrm{~Hz}, 1 \mathrm{H}), 3.56(\mathrm{dd}, J=8.0 \mathrm{~Hz}, J=2.8 \mathrm{~Hz}$, 1H), $3.15(\mathrm{~s}, 6 \mathrm{H}), 2.06-1.99(\mathrm{~m}, 1 \mathrm{H}), 1.88-1.81(\mathrm{~m}, 1 \mathrm{H}), 1.80-1.72(\mathrm{~m}, 1 \mathrm{H})$, 1.64-1.56 (m, 1H), 1.43 (, 3H), 1.40 (s, 3H), 1.39 (s, 3H), 1.35 (s, 3H), 1.32 (s, 3H), 1.29-1.23 (m, 4H), $1.21(\mathrm{~s}, 3 \mathrm{H}) ;{ }^{13} \mathrm{C} \mathrm{NMR}\left(100 \mathrm{MHz}, \mathrm{CDCl}_{3}\right): \delta 180.3,180.0,159.8$, $159.7,155.5,155.2,145.0,144.7,143.3,143.1,133.4,133.1,129.0,128.8,128.2$, $127.9,127.4,125.1,125.0,122.7,122.5,122.3,122.2,119.2,118.8,114.3,114.1$, $113.84,113.75,108.1,107.6,71.9,71.0,55.3,55.2,50.0,49.2,48.2,48.1,34.9,32.8$, 28.00, 27.96, 26.1, 26.1, 24.5, 23.7, 22.3, 22.0, 19.6, 19.2; ESI-HRMS: $m / z$ Calcd for $\mathrm{C}_{30} \mathrm{H}_{33} \mathrm{~N}_{3} \mathrm{O}_{2}+\mathrm{H}^{+}$: 468.2646, found 468.2640.

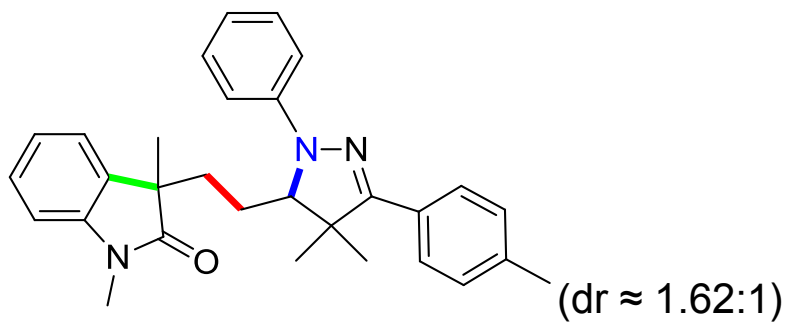

3-(2-(4, 4-dimethyl-1-phenyl-3-(p-tolyl)-4, 5-dihydro-1H-pyrazol-5-yl) ethyl)-1, 3dimethylindolin-2-one $(3 w)$

Yellow oil; (156 mg, 69\% yield); $\mathrm{R}_{f}=0.40$ (hexanes/acetone 5:1, twice); ${ }^{1} \mathrm{H}$ NMR $\left(400 \mathrm{MHz}, \mathrm{CDCl}_{3}\right): \delta 7.62(\mathrm{~d}, J=8.4 \mathrm{~Hz}, 2 \mathrm{H}), 7.56(\mathrm{~d}, J=8.4 \mathrm{~Hz}, 2 \mathrm{H}), 7.23-7.14(\mathrm{~m}$, 10H), $7.07(\mathrm{~d}, J=7.2 \mathrm{~Hz}, 1 \mathrm{H}), 7.02(\mathrm{~d}, J=8.0 \mathrm{~Hz}, 3 \mathrm{H}), 6.92(\mathrm{~d}, J=8.0 \mathrm{~Hz}, 2 \mathrm{H})$, 6.86-6.74 (m, 5H), 6.47 (d, $J=7.2 \mathrm{~Hz}, 1 \mathrm{H}), 3.79(\mathrm{~d}, J=5.6 \mathrm{~Hz}, 1 \mathrm{H}), 3.58(\mathrm{dd}, J=$ $8.4 \mathrm{~Hz}, J=2.8 \mathrm{~Hz}, 1 \mathrm{H}), 3.14(\mathrm{~s}, 6 \mathrm{H}), 2.38(\mathrm{~s}, 3 \mathrm{H}), 2.35(\mathrm{~s}, 3 \mathrm{H}), 2.02(\mathrm{td}, J=13.2 \mathrm{~Hz}$, $J=5.2 \mathrm{~Hz}, 1 \mathrm{H}), 1.89-1.72(\mathrm{~m}, 2 \mathrm{H}), 1.64-1.45(\mathrm{~m}, 2 \mathrm{H}), 1.43(\mathrm{~s}, 3 \mathrm{H}), 1.41(\mathrm{~s}, 3 \mathrm{H})$, $1.39(\mathrm{~s}, 3 \mathrm{H}), 1.36(\mathrm{~s}, 3 \mathrm{H}), 1.32(\mathrm{~s}, 3 \mathrm{H}), 1.31-1.23(\mathrm{~m}, 3 \mathrm{H}), 1.20(\mathrm{~s}, 3 \mathrm{H}) ;{ }^{13} \mathrm{C} \mathrm{NMR}$ $\left(100 \mathrm{MHz}, \mathrm{CDCl}_{3}\right): \delta 180.3,180.0,155.6,155.3,144.8,144.6,143.3,143.1,138.2$, $138.1,133.4,133.1,129.62,129.59,129.05,129.0,128.97,128.8,127.9,127.4,126.8$, $122.7,122.5,122.3,122.2,119.2,118.9,114.3,114.1,108.0,107.6,72.0,71.1,50.0$, $49.2,48.2,48.1,34.9,32.8,28.01,27.97,26.05,26.02,24.5,23.7,22.2,22.0,21.22$, 21.19, 19.5, 19.1; ESI-HRMS: $m / z$ Calcd for $\mathrm{C}_{30} \mathrm{H}_{33} \mathrm{~N}_{3} \mathrm{O}+\mathrm{H}^{+}$: 452.2696, found 452.2691 . 


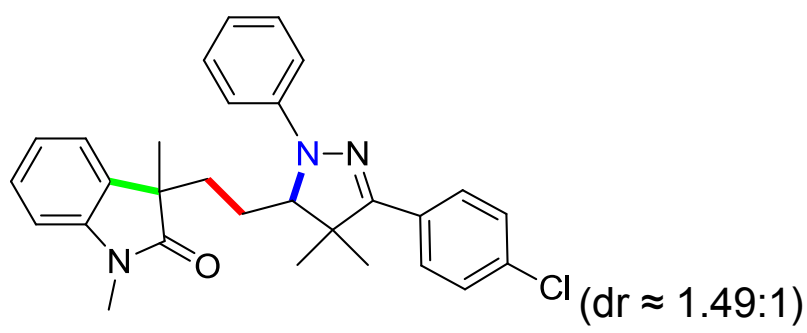

3-(2-(3-(4-chlorophenyl)-4, 4-dimethyl-1-phenyl-4, 5-dihydro-1H-pyrazol-5-yl) ethyl)-1, 3-dimethylindolin-2-one (3x)

Yellow oil; (170 mg, 72\% yield); $\mathrm{R}_{f}=0.30$ (hexanes/acetone 5:1, twice); ${ }^{1} \mathrm{H}$ NMR $\left(400 \mathrm{MHz}, \mathrm{CDCl}_{3}\right): \delta 7.66(\mathrm{~d}, J=8.8 \mathrm{~Hz}, 2 \mathrm{H}), 7.60(\mathrm{~d}, J=8.4 \mathrm{~Hz}, 2 \mathrm{H}), 7.34(\mathrm{~d}, J=$ $8.4 \mathrm{~Hz}, 2 \mathrm{H}), 7.30(\mathrm{~d}, J=8.4 \mathrm{~Hz}, 2 \mathrm{H}), 7.25-7.14(\mathrm{~m}, 6 \mathrm{H}), 7.08-7.00(\mathrm{~m}, 3 \mathrm{H})$, 6.90-6.74 (m, 8H), $6.46(\mathrm{~d}, J=7.2 \mathrm{~Hz}, 1 \mathrm{H}), 3.82(\mathrm{~d}, J=5.2 \mathrm{~Hz}, 1 \mathrm{H}), 3.61(\mathrm{dd}, J=$ $8.4 \mathrm{~Hz}, J=2.4 \mathrm{~Hz}, 1 \mathrm{H}), 3.145(\mathrm{~s}, 3 \mathrm{H}), 3.140(\mathrm{~s}, 3 \mathrm{H}), 2.02(\mathrm{td}, J=12.8 \mathrm{~Hz}, J=7.6 \mathrm{~Hz}$, $1 \mathrm{H}), 1.82(\mathrm{td}, J=13.2 \mathrm{~Hz}, J=8.8 \mathrm{~Hz}, 1 \mathrm{H}), 1.74(\mathrm{td}, J=12.4 \mathrm{~Hz}, J=3.6 \mathrm{~Hz}, 1 \mathrm{H})$, 1.61-1.41 (m, 3H), 1.38 (s, 3H), 1.35 (s, 3H), 1.32 (s, 3H), 1.31 (s, 3H), 1.28 (s, 3H), 1.27-1.23 (m, 2H), 1.20 (s, 3H); ${ }^{13} \mathrm{C}$ NMR (100 MHz, $\left.\mathrm{CDCl}_{3}\right): \delta 180.2,179.9,154.2$, $153.9,144.4,144.2,143.3,143.1,134.05,134.01,133.3,133.0,131.0,129.1,128.9$, $128.6,128.5,128.0,127.5,122.7,122.5,122.3,122.2,119.7,119.4,114.5,114.2$, 108.1, 107.6, 72.1, 71.3, 49.8, 49.0, 48.2, 48.1, 34.8, 32.7, 27.94, 27.90, 26.09, 26.06, 24.5, 23.7, 22.2, 22.0, 19.4, 19.1; ESI-HRMS: $m / z$ Calcd for $\mathrm{C}_{29} \mathrm{H}_{30} \mathrm{ClN}_{3} \mathrm{O}+\mathrm{H}^{+}$: 472.2150, found 472.2141 .

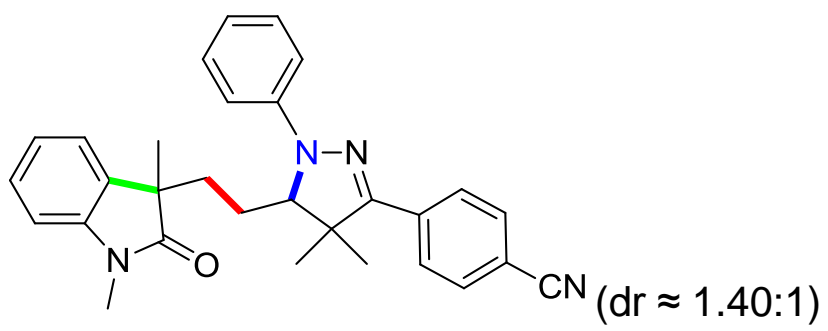

4-(5-(2-(1, 3-dimethyl-2-oxoindolin-3-yl) ethyl)-4, 4-dimethyl-1-phenyl-4, 5dihydro-1H-pyrazol-3-yl) benzonitrile (3y)

Yellow oil; (173 mg, 75\% yield); $\mathrm{R}_{f}=0.30$ (hexanes/acetone 3:1, twice); ${ }^{1} \mathrm{H}$ NMR $\left(400 \mathrm{MHz}, \mathrm{CDCl}_{3}\right): \delta 7.82(\mathrm{~d}, J=8.8 \mathrm{~Hz}, 2 \mathrm{H}), 7.79(\mathrm{~d}, J=8.8 \mathrm{H}, 2 \mathrm{H}), 7.51(\mathrm{~d}, J=$ 16.0 Hz, $J=8.8 \mathrm{~Hz}, 4 \mathrm{H}), 7.72-7.14(\mathrm{~m}, 7 \mathrm{H}), 7.08-7.03(\mathrm{~m}, 3 \mathrm{H}), 6.93-6.75(\mathrm{~m}, 7 \mathrm{H})$, $6.45(\mathrm{~d}, J=7.2 \mathrm{~Hz}, 1 \mathrm{H}), 3.89(\mathrm{~d}, J=5.2 \mathrm{~Hz}, 1 \mathrm{H}), 3.70(\mathrm{dd}, J=8.4 \mathrm{~Hz}, J=2.4 \mathrm{~Hz}$, $1 \mathrm{H}), 3.15$ (s, 3H), 3.14 (s, 3H), 2.04-1.98 (m, 1H), 1.86-1.81 (m, 1H), 1.79-1.73 (m, 1H), 1.58-1.51 (m, 1H), $1.45(\mathrm{~s}, 3 \mathrm{H}), 1.41(\mathrm{~s}, 3 \mathrm{H}), 1.37(\mathrm{~s}, 3 \mathrm{H}), 1.36(\mathrm{~s}, 3 \mathrm{H}) 1.31(\mathrm{~s}$, 
$3 \mathrm{H}), 1.30-1.22(\mathrm{~m}, 4 \mathrm{H}), 1.20(\mathrm{~s}, 3 \mathrm{H}) ;{ }^{13} \mathrm{C} \mathrm{NMR}\left(100 \mathrm{MHz}, \mathrm{CDCl}_{3}\right): \delta 180.1,179.8$, $152.5,152.3,143.6,143.5,143.2,143.0,137.0,133.2$, 132.8, 132.1, 132.03, 131.96, 129.1, 128.9, 128.0, 127.5, 126.6, 122.7, 122.6, 122.5, 122.14, 122.12, 120.2, 120.0, 118.92, 118.88, 114.5, 114.4, 110.84, 110.79, 108.1, 107.7, 72.4, 71.7, 49.4, 48.6, 48.1, $48.0,34.7,32.5,27.94,27.89,26.1,26.0,24.5,23.7,22.12,22.07,19.3,19.0$; ESI-HRMS: $m / z$. Calcd for $\mathrm{C}_{30} \mathrm{H}_{30} \mathrm{~N}_{4} \mathrm{O}+\mathrm{H}^{+}$: 463.2492, found 463.2488.

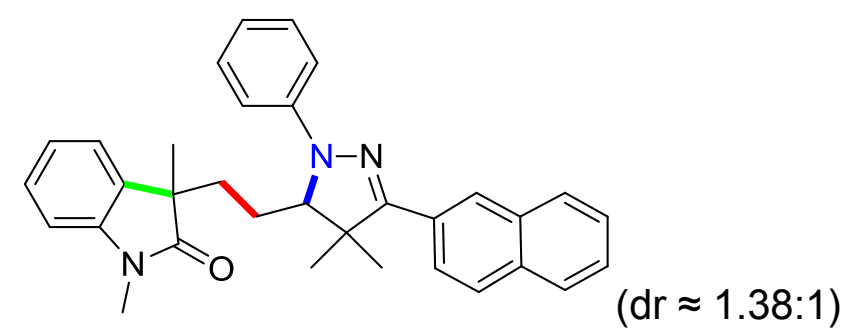

3-(2-(4, 4-dimethyl-3-(naphthalen-2-yl)-1-phenyl-4, 5-dihydro-1H-pyrazol-5-yl) ethyl)-1, 3-dimethylindolin-2-one (3z)

Yellow oil; (144 mg, 59\% yield); $\mathrm{R}_{f}=0.30$ (hexanes/acetone 5:1); ${ }^{1} \mathrm{H}$ NMR (400 $\mathrm{MHz}_{\mathrm{CDCl}}$ ): $\delta 8.09$ (s, 1H), $8.04(\mathrm{~s}, 1 \mathrm{H}), 8.00(\mathrm{dd}, J=8.8 \mathrm{~Hz}, J=1.6 \mathrm{~Hz}, 1 \mathrm{H}), 7.93$ $(\mathrm{dd}, J=8.8 \mathrm{~Hz}, J=1.6 \mathrm{~Hz}, 1 \mathrm{H}), 7.87-7.78(\mathrm{~m}, 6 \mathrm{H}), 7.51-7.45(\mathrm{~m}, 4 \mathrm{H}), 7.24-7.16(\mathrm{~m}$, 7H), $7.06(\mathrm{dd}, J=8.4 \mathrm{~Hz}, J=4.0 \mathrm{~Hz}, 3 \mathrm{H}), 6.95(\mathrm{~d}, J=8.0 \mathrm{~Hz}, 2 \mathrm{H}), 6.89-6.75(\mathrm{~m}$, $5 \mathrm{H}), 6.48(\mathrm{~d}, J=7.2 \mathrm{~Hz}, 1 \mathrm{H}), 3.87(\mathrm{~d}, J=5.2 \mathrm{~Hz}, 1 \mathrm{H}), 3.67(\mathrm{dd}, J=8.0 \mathrm{~Hz}, J=2.4$ $\mathrm{Hz}, 1 \mathrm{H}), 3.15$ (s, 3H), 3.147 (s, 3H), 2.08 (td, $J=12.0 \mathrm{~Hz}, J=4.8 \mathrm{~Hz}, 1 \mathrm{H}), 1.92$ (td, $J$ $=12.0 \mathrm{~Hz}, J=4.0 \mathrm{~Hz}, 1 \mathrm{H}), 1.85-1.63(\mathrm{~m}, 2 \mathrm{H}), 1.61(\mathrm{~s}, 3 \mathrm{H}), 1.57(\mathrm{~s}, 3 \mathrm{H}), 1.52(\mathrm{~s}, 3 \mathrm{H})$, $1.48(\mathrm{~s}, 3 \mathrm{H}), 1.47(\mathrm{~s}, 3 \mathrm{H}), 1.45-1.26(\mathrm{~m}, 4 \mathrm{H}), 1.24(\mathrm{~s}, 3 \mathrm{H}) ;{ }^{13} \mathrm{C}$ NMR $(100 \mathrm{MHz}$, $\left.\mathrm{CDCl}_{3}\right): 180.3,180.0,155.1,154.8,144.5,144.3,143.3,143.1,133.3,133.14,133.06$, 133.0, 130.0, 129.9, 129.1, 128.9, 128.24, 128.21, 127.93, 127.87, 127.59, 127.56, $127.4,126.24,126.22,126.18,125.19,125.16,125.15,122.7,122.5,122.3,122.2$, 119.5, 119.2, 114.4, 114.2, 108.1, 107.6, 72.1, 71.3, 49.9, 49.1, 48.2, 48.1, 34.8, 32.7, 29.6, 28.2, 28.1, 26.0, 24.5, 23.7, 22.1, 19.7, 19.4, 14.1; ESI-HRMS: $m / z$ Calcd for $\mathrm{C}_{33} \mathrm{H}_{33} \mathrm{~N}_{3} \mathrm{O}+\mathrm{H}^{+}:$488.2696, found 488.2690.

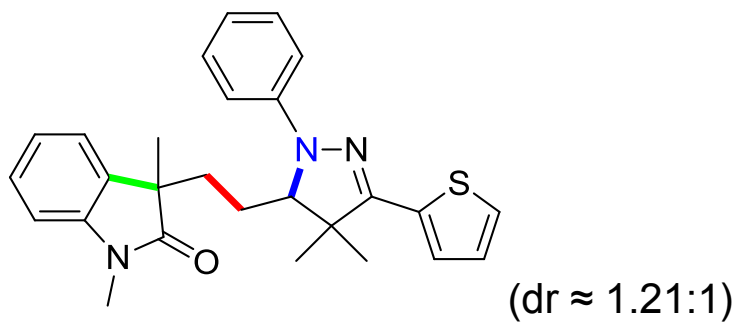


3-(2-(4, 4-dimethyl-1-phenyl-3-(thiophen-2-yl)-4, 5-dihydro-1H-pyrazol-5-yl) ethyl)1, 3-dimethylindolin-2-one (3aa)

Yellow oil; (200 mg, 86\% yield); $\mathrm{R}_{f}=0.35$ (hexanes/acetone 5:1); ${ }^{1} \mathrm{H}$ NMR (400 $\left.\mathrm{MHz}, \mathrm{CDCl}_{3}\right): \delta 7.29-7.14(\mathrm{~m}, 12 \mathrm{H}), 7.08-6.98(\mathrm{~m}, 5 \mathrm{H}), 6.88-6.74(\mathrm{~m}, 6 \mathrm{H}), 6.50(\mathrm{~d}$, $J=7.6 \mathrm{~Hz}, 1 \mathrm{H}), 3.82(\mathrm{~d}, J=5.2 \mathrm{~Hz}, 1 \mathrm{H}), 3.61(\mathrm{dd}, J=8.8 \mathrm{~Hz}, J=2.4 \mathrm{~Hz}, 1 \mathrm{H}), 3.15$ (s, 6H), $2.04(\mathrm{td}, J=12.0 \mathrm{~Hz}, J=5.2 \mathrm{~Hz}, 1 \mathrm{H}), 1.88(\mathrm{td}, J=12.0 \mathrm{~Hz}, J=4.4 \mathrm{~Hz}, 1 \mathrm{H})$, $1.77(\mathrm{td}, J=12.0 \mathrm{~Hz}, J=4.8 \mathrm{~Hz}, 1 \mathrm{H}), 1.64-1.51(\mathrm{~m}, 2 \mathrm{H}), 1.49(\mathrm{~s}, 3 \mathrm{H}), 1.47(\mathrm{~s}, 3 \mathrm{H})$, $1.40(\mathrm{~s}, 3 \mathrm{H}), 1.39$ (s, 3H), $1.34(\mathrm{~s}, 3 \mathrm{H}), 1.32-1.23(\mathrm{~m}, 3 \mathrm{H}), 1.22(\mathrm{~s}, 3 \mathrm{H}) ;{ }^{13} \mathrm{C} \mathrm{NMR}$ $\left(100 \mathrm{MHz}, \mathrm{CDCl}_{3}\right): \delta 180.4,180.0,151.3,151.0,144.5,144.2,143.4,143.2,135.72$, 135.68, 133.4, 133.1, 129.1, 128.9, 128.0, 127.5, 127.4, 127.3, 125.8, 124.6, 124.5, $122.7,122.6,122.4,122.3,119.6,119.3,114.5,114.3,108.2,107.7,71.9,71.0,50.2$, 49.4, 48.3, 48.2, 35.0, 32.8, 29.7, 28.0, 27.9, 26.1, 24.5, 23.8, 22.4, 22.2, 19.7, 19.3; ESI-HRMS: $m / z$ Calcd for $\mathrm{C}_{27} \mathrm{H}_{29} \mathrm{~N}_{3} \mathrm{OS}+\mathrm{Na}^{+}: 466.1924$, found 466.1917 .

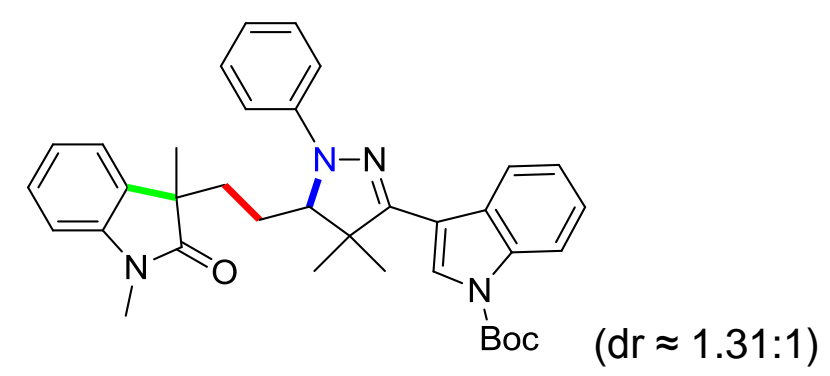

tert-butyl 3-(5-(2-(1, 3-dimethyl-2-oxoindolin-3-yl) ethyl)-4, 4-dimethyl-1-phenyl-4, 5-dihydro-1H-pyrazol-3-yl)-1H-indole-1-carboxylate (3ab)

Yellow oil; (190 mg, 66\% yield); $\mathrm{R}_{f}=0.30$ (hexanes/acetone 5:1, twice); ${ }^{1} \mathrm{H}$ NMR $\left(400 \mathrm{MHz}, \mathrm{CDCl}_{3}\right): \delta 8.51(\mathrm{~d}, J=6.8 \mathrm{~Hz}, 1 \mathrm{H}), 8.42(\mathrm{~d}, J=7.2 \mathrm{~Hz}, 1 \mathrm{H}), 8.13-8.09(\mathrm{~m}$, 2H), $7.88(\mathrm{~s}, 1 \mathrm{H}), 7.84(\mathrm{~s}, 1 \mathrm{H}), 7.39-7.32(\mathrm{~m}, 4 \mathrm{H}), 7.28-7.15(\mathrm{~m}, 9 \mathrm{H}), 7.08-7.05(\mathrm{~m}$, $3 \mathrm{H}), 6.95(\mathrm{~d}, J=8.0 \mathrm{~Hz}, 1 \mathrm{H}), 6.88-6.73(\mathrm{~m}, 4 \mathrm{H}), 6.47(\mathrm{~d}, J=7.2 \mathrm{~Hz}, 1 \mathrm{H}), 3.80(\mathrm{~d}, J$ $=6.0 \mathrm{~Hz}, 1 \mathrm{H}), 3.60(\mathrm{~d}, J=6.0 \mathrm{~Hz}, 1 \mathrm{H}), 3.14(\mathrm{~s}, 3 \mathrm{H}), 3.13(\mathrm{~s}, 3 \mathrm{H}), 2.05(\mathrm{td}, J=13.2$ $\mathrm{Hz}, J=5.2 \mathrm{~Hz}, 1 \mathrm{H}), 1.91-1.76(\mathrm{~m}, 3 \mathrm{H}), 1.76(\mathrm{~s}, 9 \mathrm{H}), 1.71(\mathrm{~s}, 9 \mathrm{H}), 1.70-1.58(\mathrm{~m}, 2 \mathrm{H})$, $1.52(\mathrm{~s}, 3 \mathrm{H}), 1.43(\mathrm{~s}, 3 \mathrm{H}), 1.41$ (s, 3H), 1.35 (s, 3H), 1.32 (s, 3H), 1.26-1.23 (s, 2H), $1.17(\mathrm{~s}, 3 \mathrm{H}) ;{ }^{13} \mathrm{C}$ NMR $\left(100 \mathrm{MHz}, \mathrm{CDCl}_{3}\right): \delta 180.3,180.0,151.2,150.9,149.6,144.8$, 144.6, 143.3, 143.1, 135.2, 135.1, 133.4, 133.1, 129.2, 129.1, 128.9, 127.9, 127.4, $125.0,123.5,123.4,123.1,122.7,122.5,122.3,122.2,119.2,119.0,114.8,114.7$, $114.2,114.1,112.9,112.8,108.1,107.6,84.24,84.21,70.6,69.7,50.5,49.7,48.3$, 
$48.2,35.0,32.7,28.2,28.1,26.10,26.06,24.6,23.8,22.2,22.1,20.0,19.6$; ESI-HRMS: $m / z$ Calcd for $\mathrm{C}_{36} \mathrm{H}_{40} \mathrm{~N}_{4} \mathrm{O}_{3}+\mathrm{H}^{+}:$577.3173, found 577.3161.

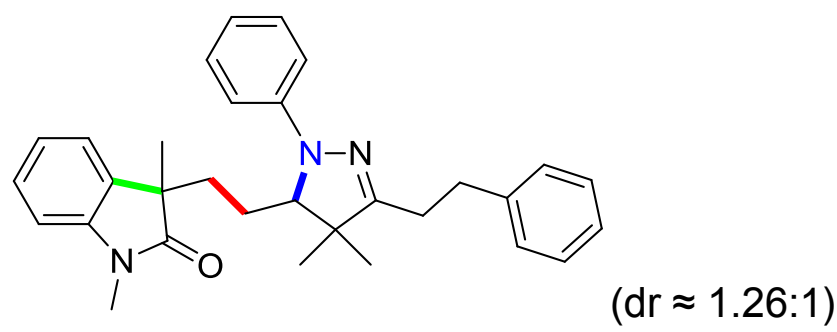

3-(2-(4, 4-dimethyl-3-phenethyl-1-phenyl-4, 5-dihydro-1H-pyrazol-5-yl) ethyl)-1, 3dimethylindolin-2-one (3ac)

Yellow oil; (188 mg, 81\% yield); $\mathrm{R}_{f}=0.35$ (hexanes/acetone 5:1, twice); ${ }^{1} \mathrm{H}$ NMR $\left(400 \mathrm{MHz}, \mathrm{CDCl}_{3}\right): \delta 7.29-7.08(\mathrm{~m}, 18 \mathrm{H}), 6.91(\mathrm{~d}, J=8.8 \mathrm{~Hz}, 2 \mathrm{H}), 6.87(\mathrm{~d}, J=7.6$ $\mathrm{Hz}, 1 \mathrm{H}), 6.82-6.76(\mathrm{~m}, 6 \mathrm{H}), 6.60(\mathrm{~d}, J=7.2 \mathrm{~Hz}, 1 \mathrm{H}), 3.56(\mathrm{~d}, J=6.0 \mathrm{~Hz}, 1 \mathrm{H}), 3.30$ $(\mathrm{dd}, J=9.2 \mathrm{~Hz}, J=2.8 \mathrm{~Hz}, 1 \mathrm{H}), 3.16(\mathrm{~s}, 3 \mathrm{H}), 3.15(\mathrm{~s}, 3 \mathrm{H}), 3.05-2.95(\mathrm{~m}, 4 \mathrm{H})$, $2.54-2.44(\mathrm{~m}, 4 \mathrm{H}), 2.02(\mathrm{td}, J=13.2 \mathrm{~Hz}, J=5.2 \mathrm{~Hz}, 1 \mathrm{H}), 1.83-1.70(\mathrm{~m}, 2 \mathrm{H})$, 1.64-1.56 (m, 2H), 1.34 (s, 3H), 1.32-1.26 (m, 2H), $1.24(\mathrm{~s}, 3 \mathrm{H}), 1.21-1.16(\mathrm{~m}, 1 \mathrm{H})$, $1.12(\mathrm{~s}, 3 \mathrm{H}), 1.10(\mathrm{~s}, 3 \mathrm{H}), 1.07(\mathrm{~s}, 3 \mathrm{H}), 1.06(\mathrm{~s}, 3 \mathrm{H}) ;{ }^{13} \mathrm{C} \mathrm{NMR}\left(100 \mathrm{MHz}, \mathrm{CDCl}_{3}\right): \delta$ $180.3,180.1,159.2,158.6,146.0,145.5,143.4,143.1,141.99,141.96,133.3,133.1$, $129.0,128.7,128.4,128.3,127.9,127.5,125.9,122.6,122.5,122.4,122.2,119.0$, 118.6, 114.4, 114.0, 108.1, 107.7, 70.6, 69.3, 50.5, 49.8, 48.24, 48.22, 35.0, 33.2, 32.6, 32.5, 28.0, 27.96, 26.7, 26.1, 26.0, 24.4, 23.8, 22.5, 22.3, 18.7, 18.3; ESI-HRMS: $\mathrm{m} / \mathrm{z}$ Calcd for $\mathrm{C}_{31} \mathrm{H}_{35} \mathrm{~N}_{3} \mathrm{O}+\mathrm{H}^{+}$: 466.2853, found 466.2845.

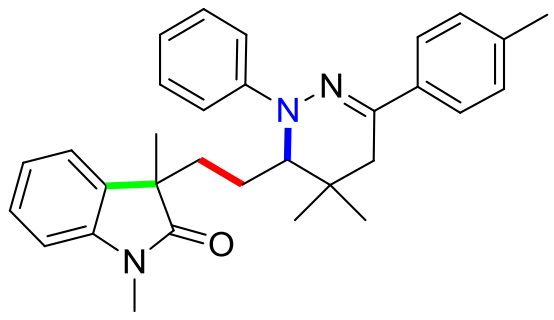

$$
(\mathrm{dr} \approx 1.05: 1)
$$

3-(2-(4, 4-dimethyl-2-phenyl-6-(p-tolyl)-2, 3, 4, 5-tetrahydropyridazin-3-yl) ethyl)-1, 3-dimethylindolin-2-one (5)

Yellow oil; (98 mg, $42 \%$ yield); $\mathrm{R}_{f}=0.35$ (hexanes/acetone 5:1, twice); ${ }^{1} \mathrm{H}$ NMR (400 $\left.\mathrm{MHz}, \mathrm{CDCl}_{3}\right): \delta 7.68(\mathrm{~d}, J=8.4 \mathrm{~Hz}, 2 \mathrm{H}), 7.61(\mathrm{~d}, J=8.4 \mathrm{~Hz}, 2 \mathrm{H}), 7.31-7.27(\mathrm{~m}, 5 \mathrm{H})$, 7.24-7.10 (m, 9H), 7.00-6.92 (m, 3H), 6.88-6.73 (m, 5H), $3.56(\mathrm{t}, J=6.2 \mathrm{~Hz}, 1 \mathrm{H})$, $3.50(\mathrm{t}, J=5.4 \mathrm{~Hz}, 1 \mathrm{H}), 3.16(\mathrm{~s}, 3 \mathrm{H}), 3.12(\mathrm{~s}, 3 \mathrm{H}), 2.36(\mathrm{~s}, 6 \mathrm{H}), 2.27(\mathrm{~d}, J=2.8 \mathrm{~Hz}$, 
2H), $2.15(\mathrm{~d}, J=3.6 \mathrm{~Hz}, 2 \mathrm{H}), 2.08-1.98(\mathrm{~m}, 2 \mathrm{H}), 1.86-1.77(\mathrm{~m}, 2 \mathrm{H}), 1.58-1.25(\mathrm{~m}$, 4H), $1.26(\mathrm{~s}, 6 \mathrm{H}), 1.05(\mathrm{~s}, 3 \mathrm{H}), 1.04(\mathrm{~s}, 3 \mathrm{H}), 0.82$ (s, 3H), $0.81(\mathrm{~s}, 3 \mathrm{H}) ;{ }^{13} \mathrm{C}$ NMR (100 $\left.\mathrm{MHz}, \mathrm{CDCl}_{3}\right): \delta 180.3,180.0,147.7,147.4,143.1,143.0,139.4,138.5,137.3,136.1$, 135.9, 133.6, 133.0, 129.0, 128.9, 128.85, 128.78, 127.7, 127.6, 124.4, 122.6, 122.4, 122.29, 122.27, 119.3, 119.2, 114.0, 113.9, 107.9, 107.8, 60.92, 60.87, 48.4, 48.0, 36.3, 36.2, 33.2, 33.0, 30.33, 30.27, 28.3, 28.0, 27.2, 27.1, 26.1, 25.1, 24.9, 24.4, 23.4, 21.1; ESI-HRMS: $m / z$ Calcd for $\mathrm{C}_{31} \mathrm{H}_{35} \mathrm{~N}_{3} \mathrm{O}+\mathrm{H}^{+}: 466.2853$, found 466.2846 . 

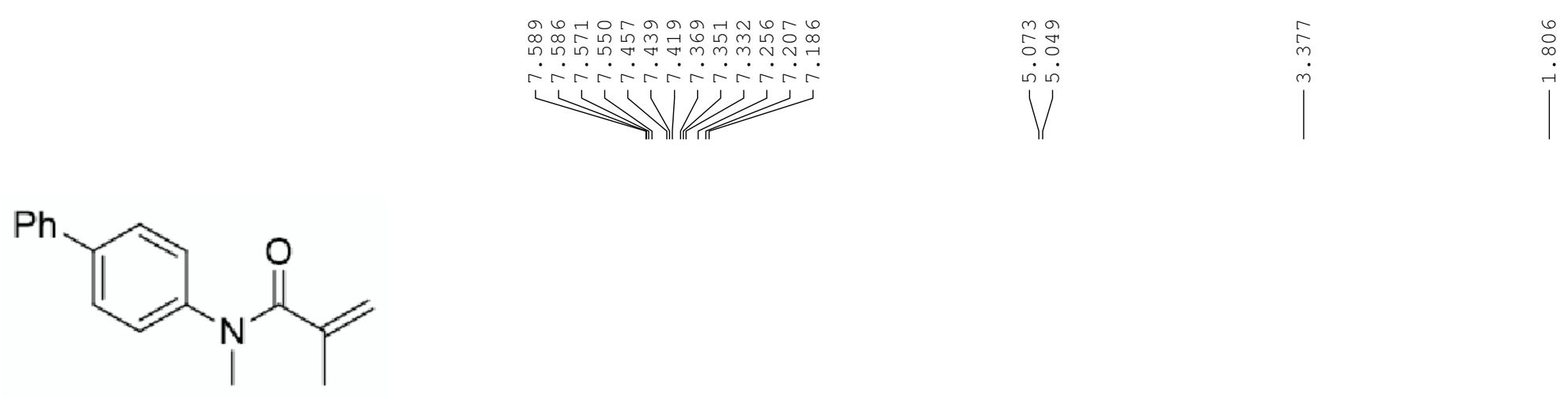

compound (1i)

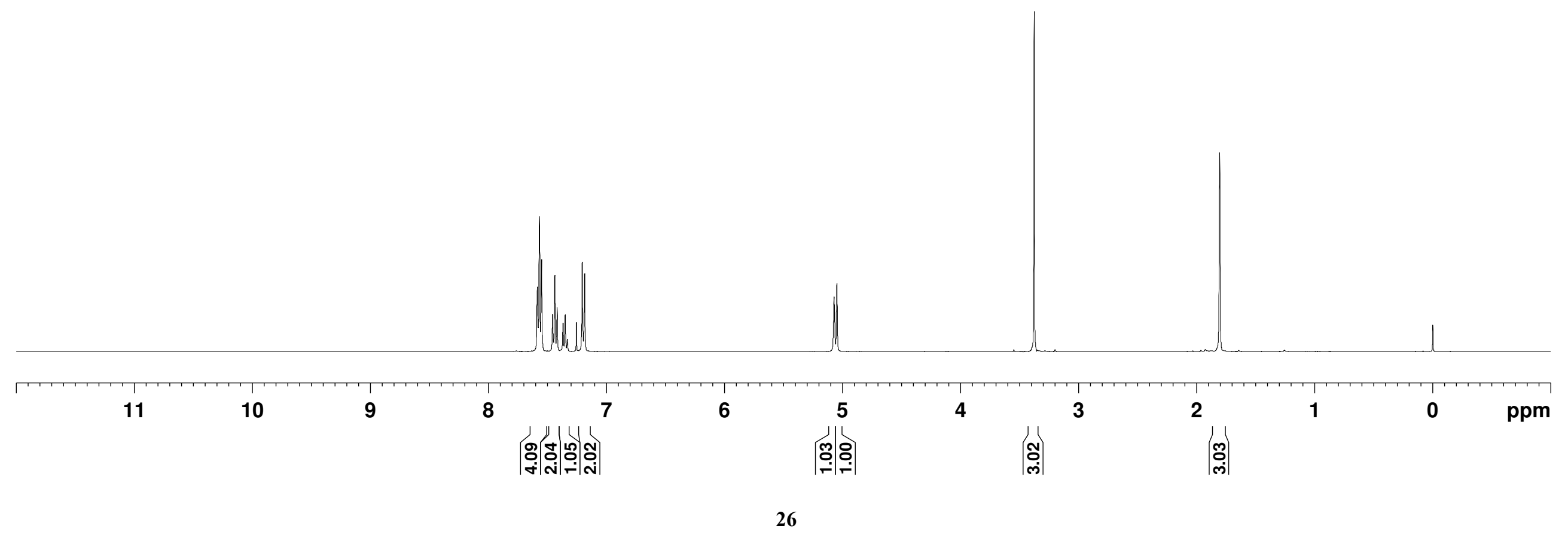



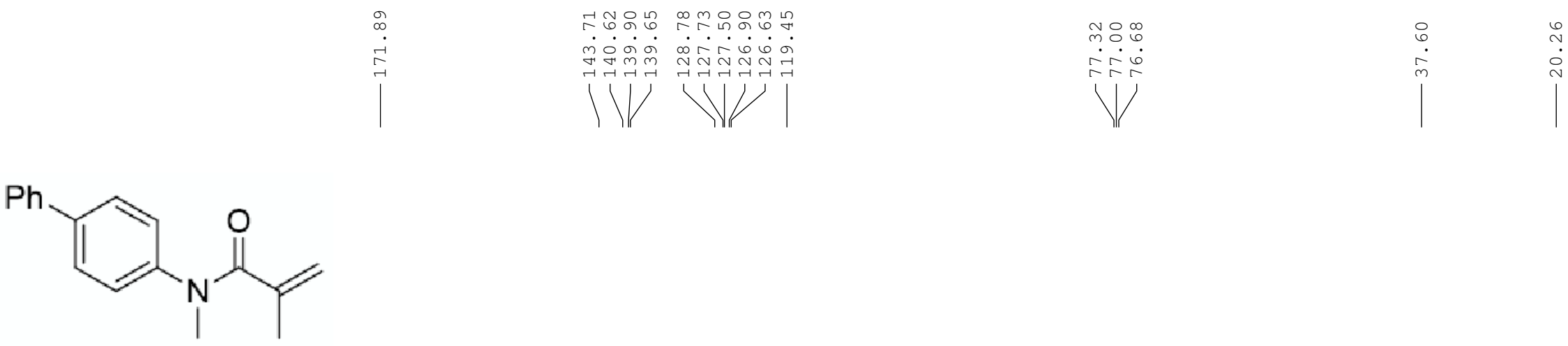

compound (1i)

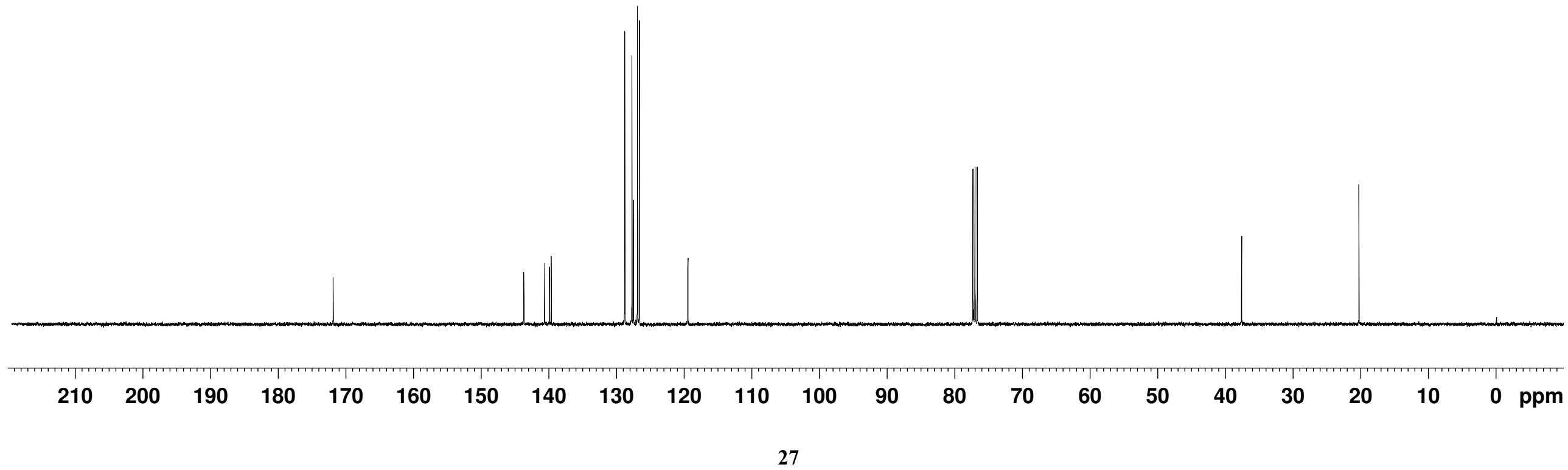



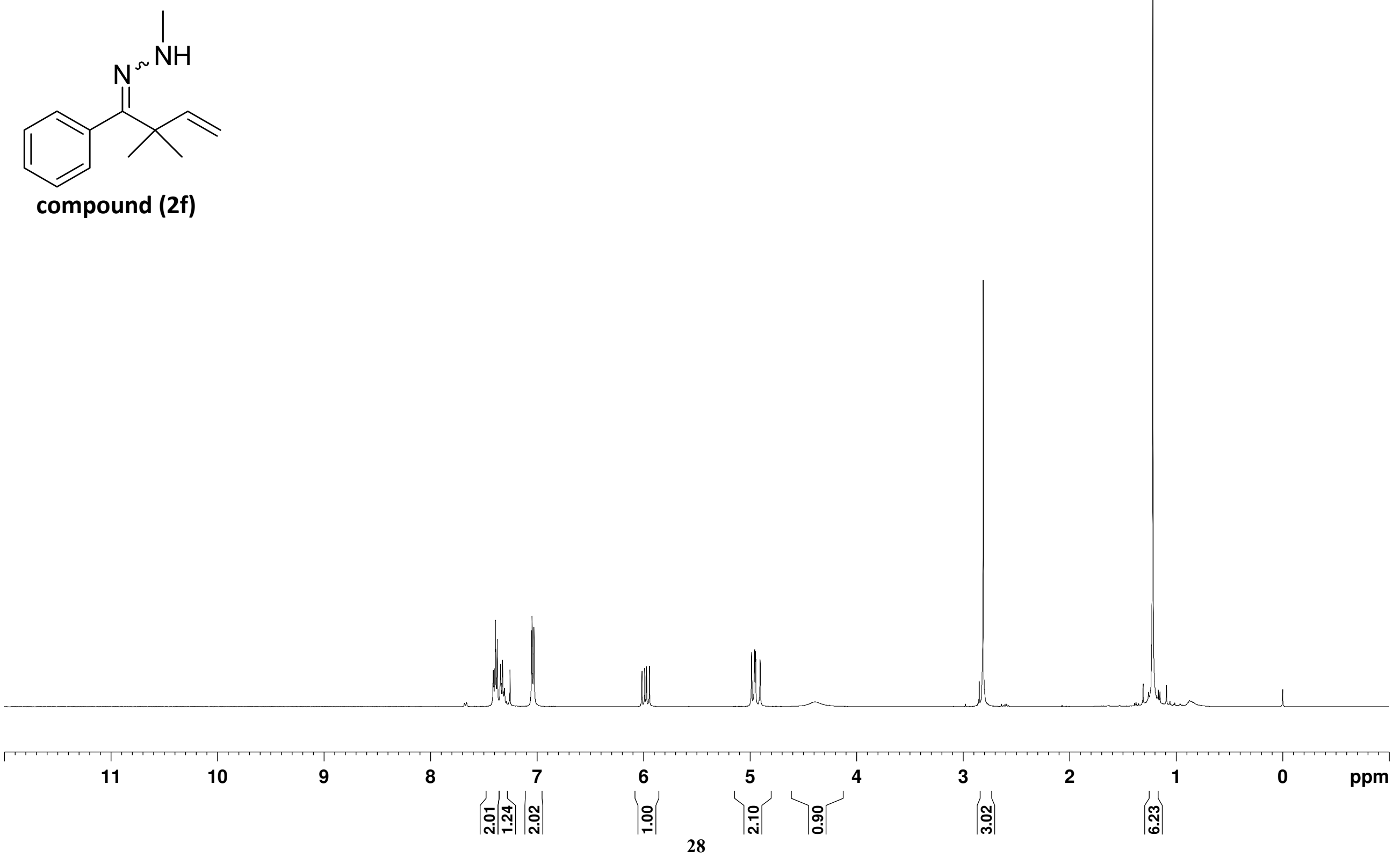
<smiles>C=CC(C)(C)C(=NNC)c1ccccc1</smiles>

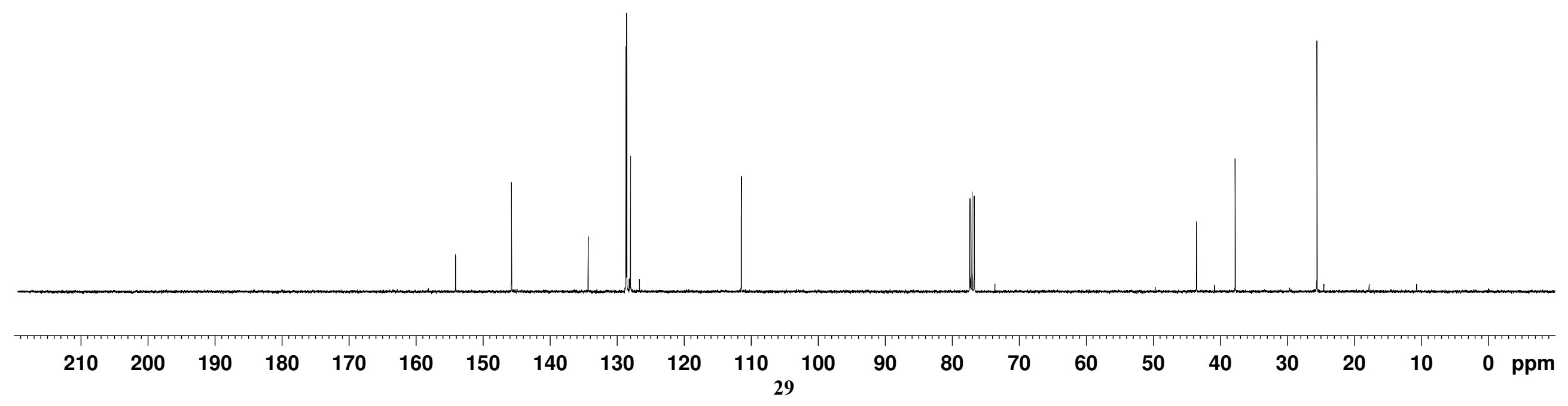




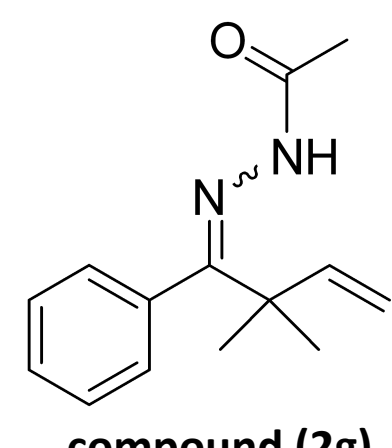

compound $(2 \mathrm{~g})$

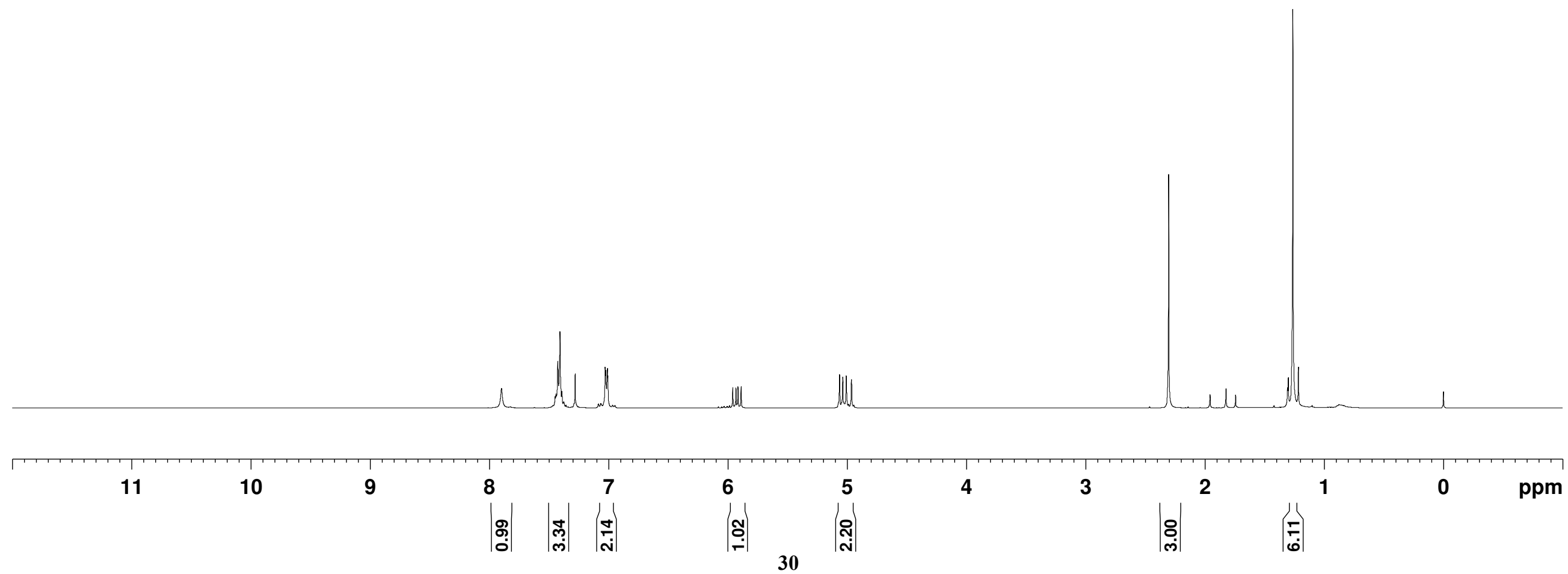




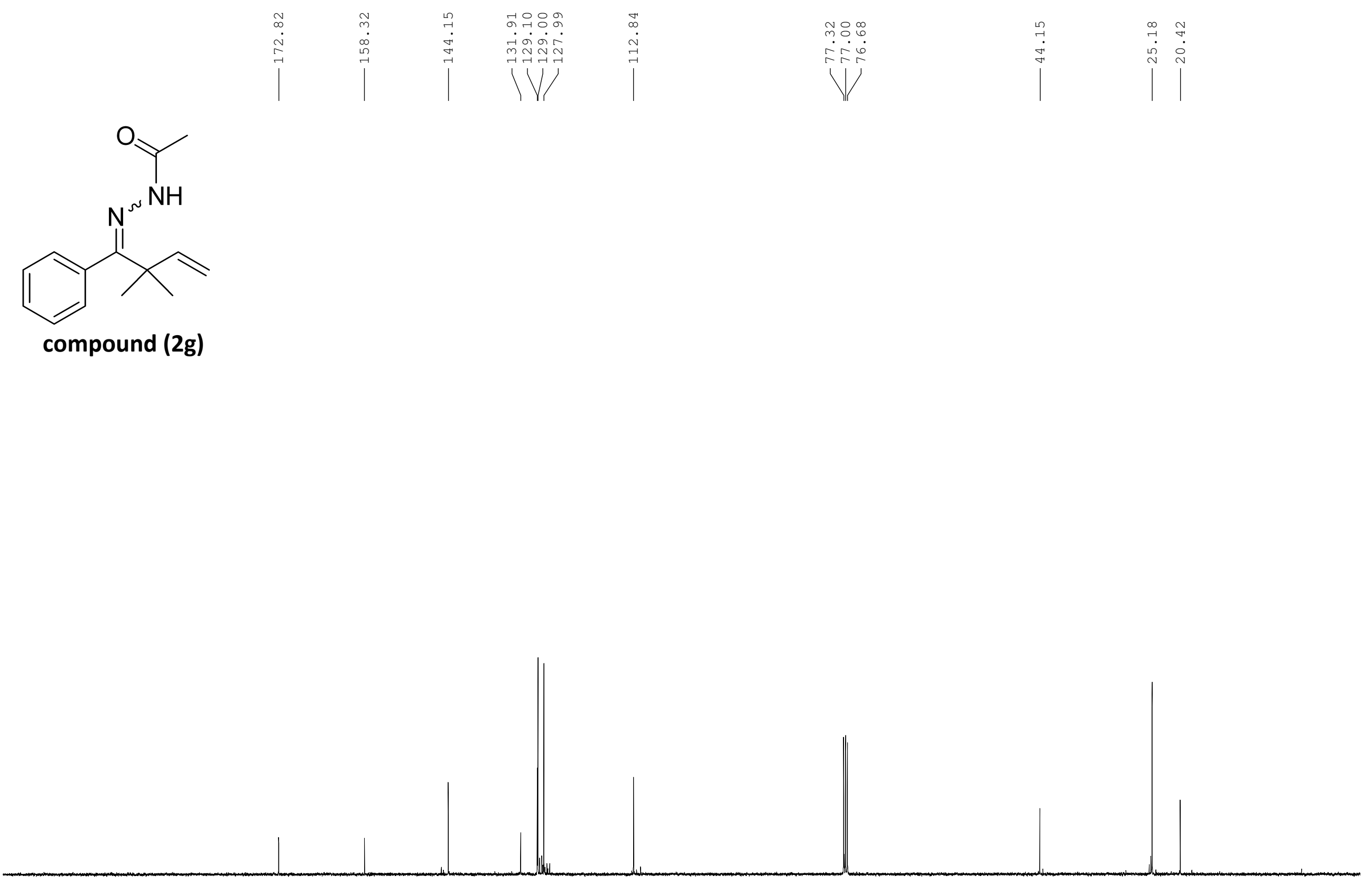



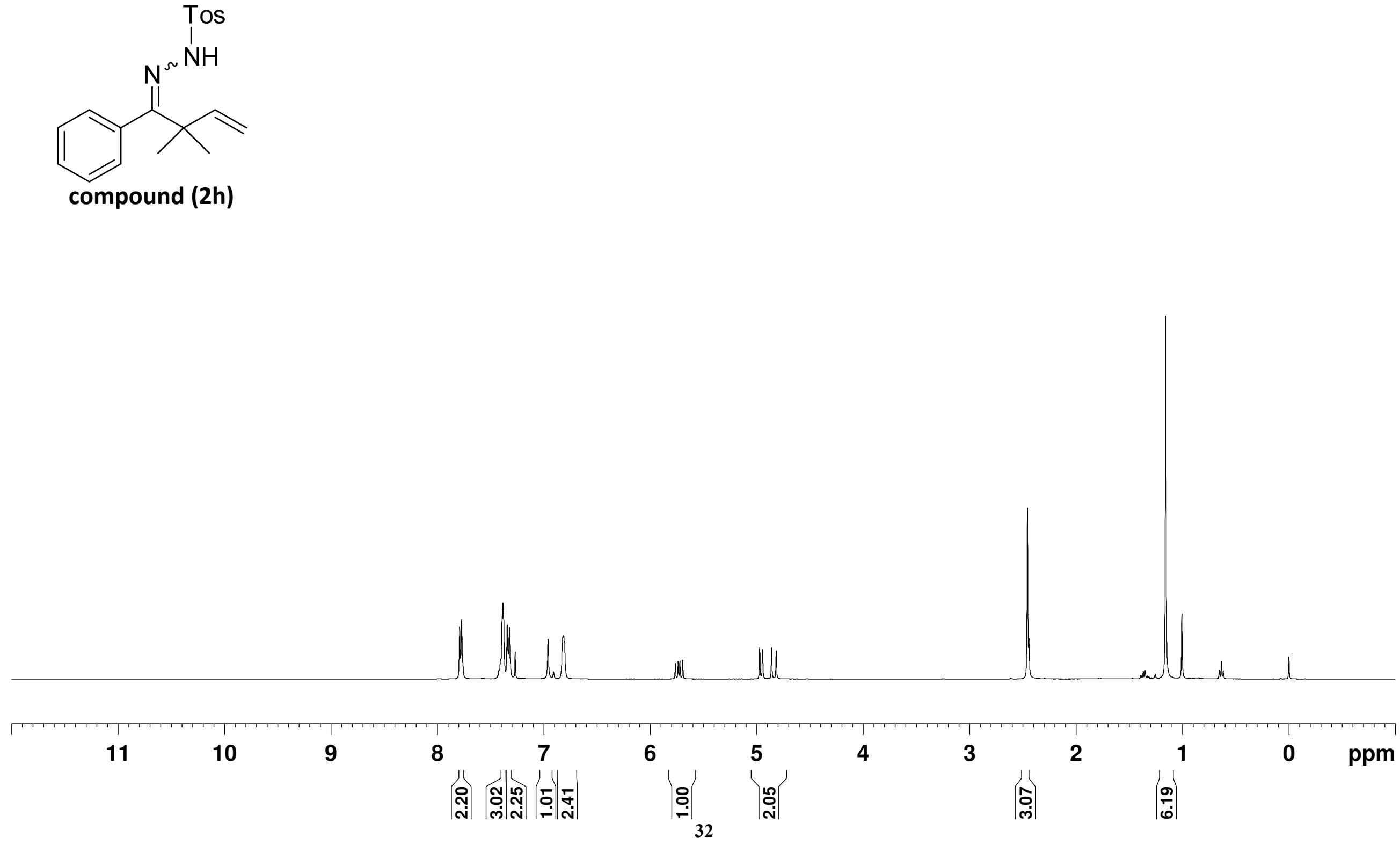

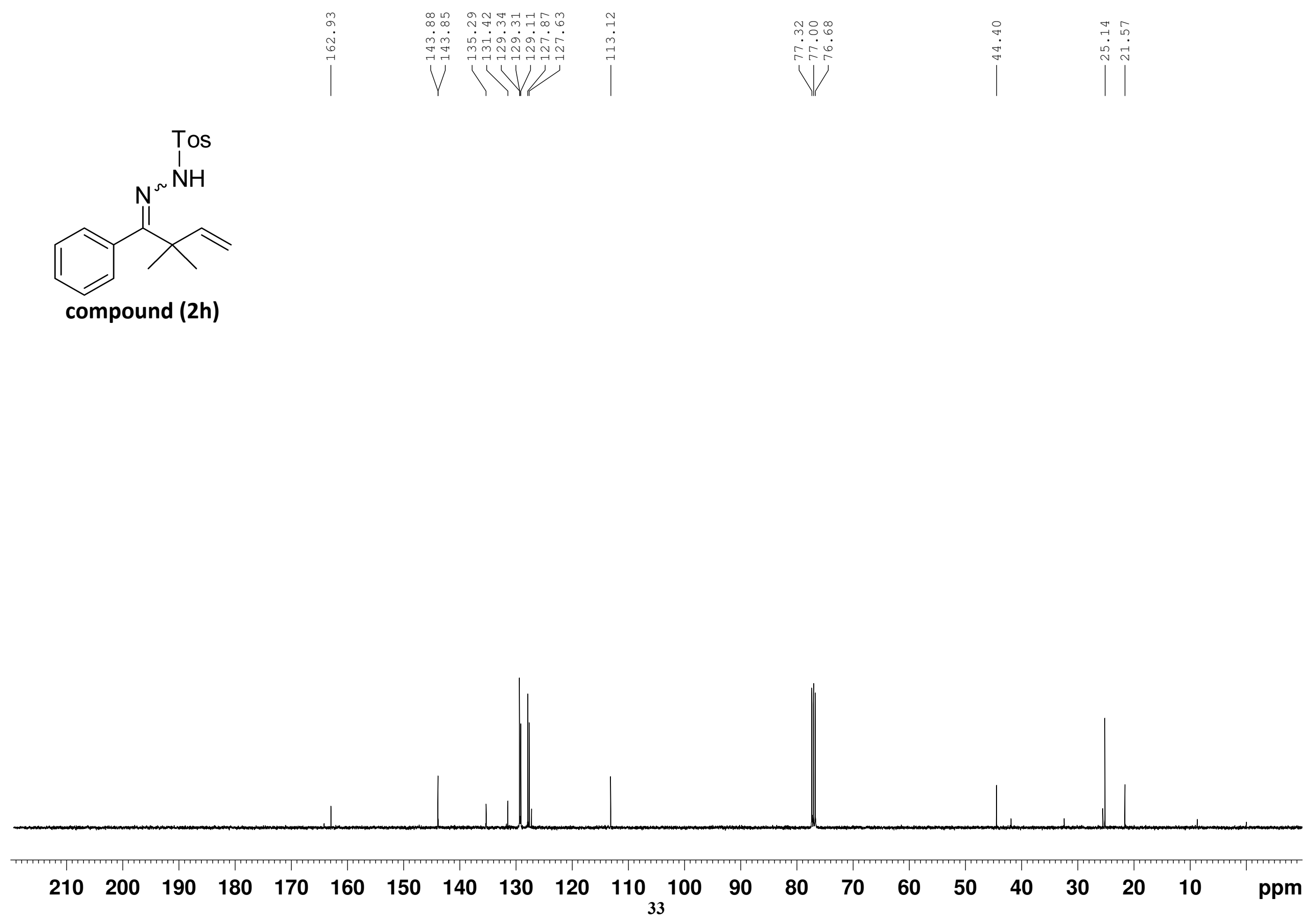

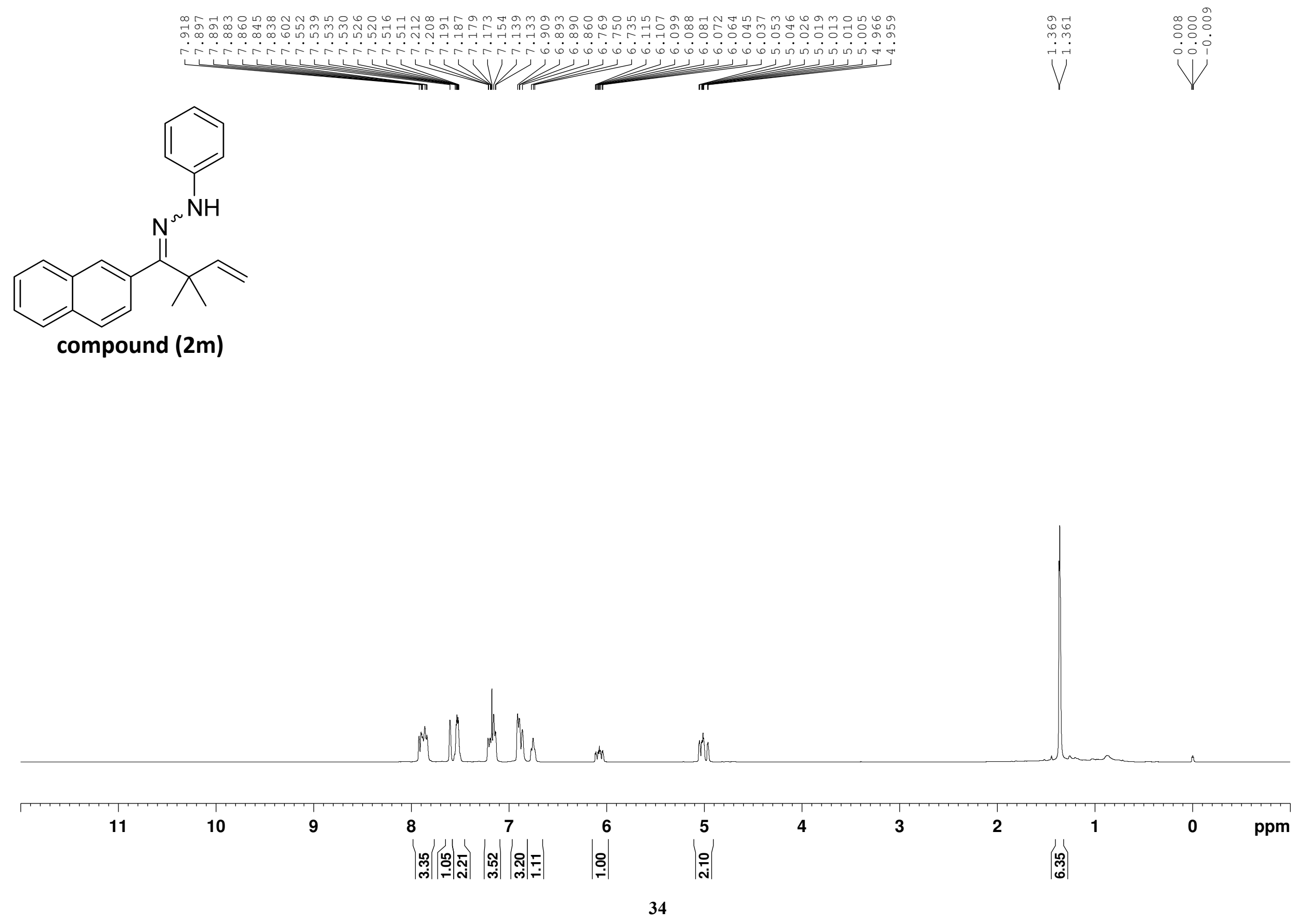

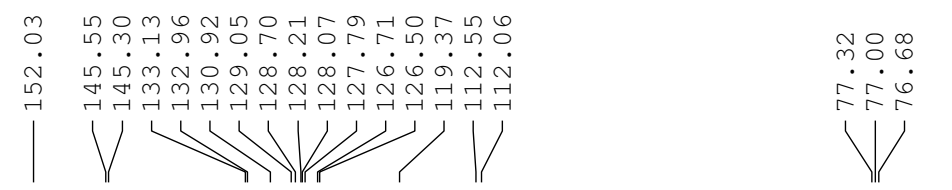

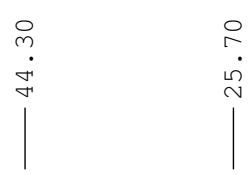
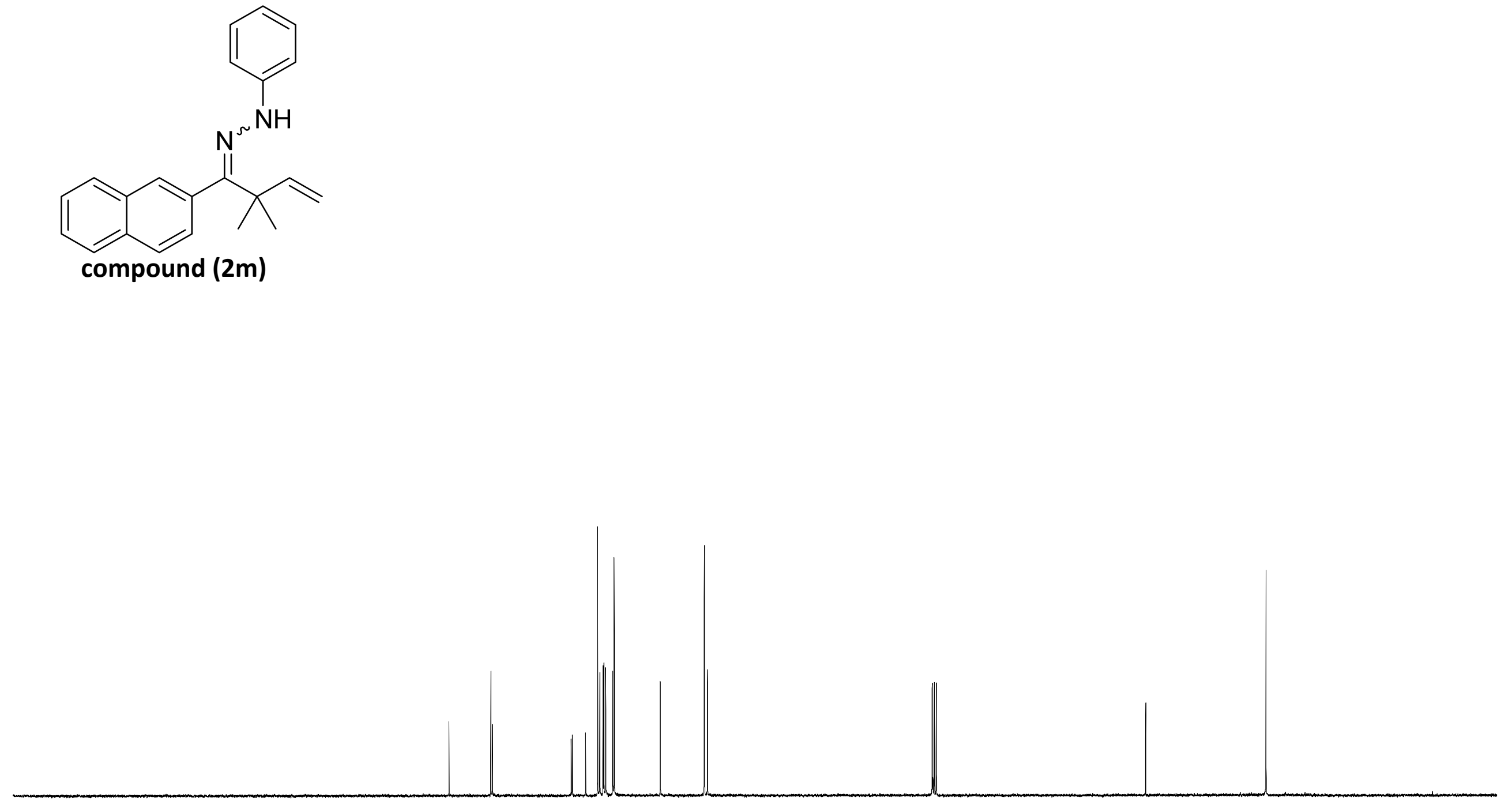


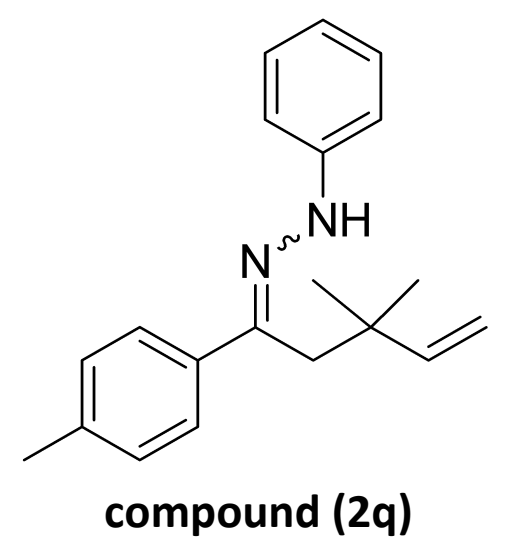

\section{compound (2q)}

(1)
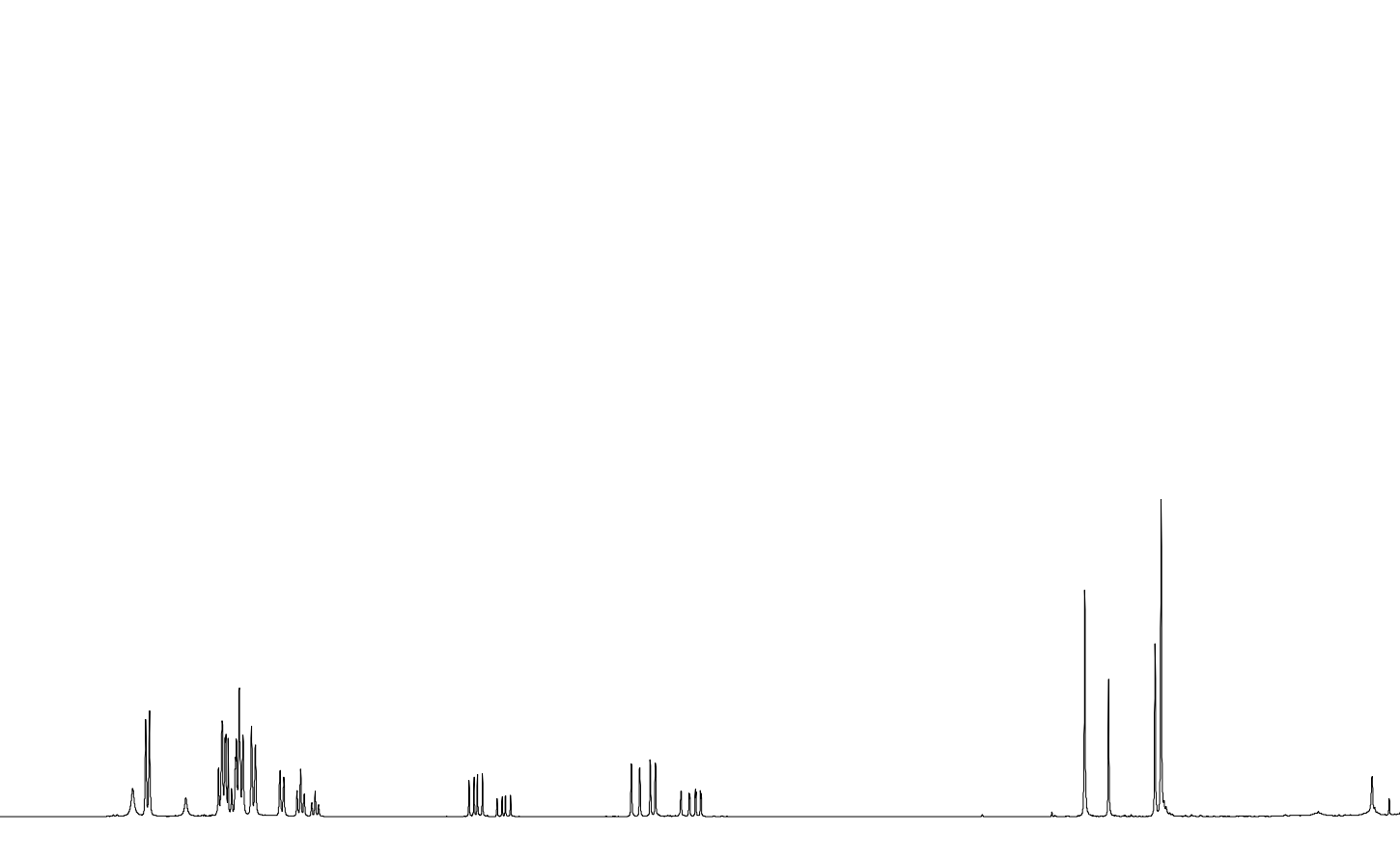

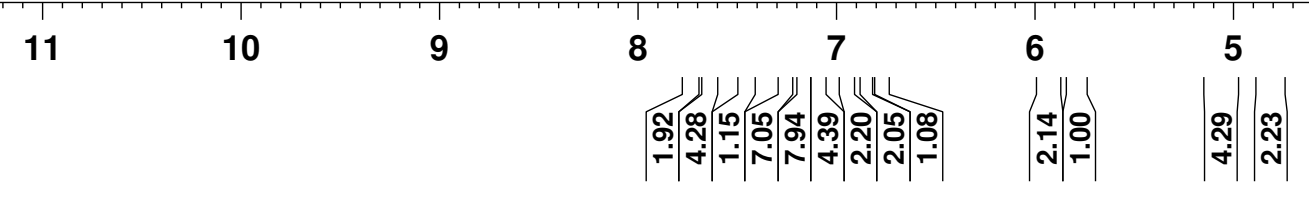




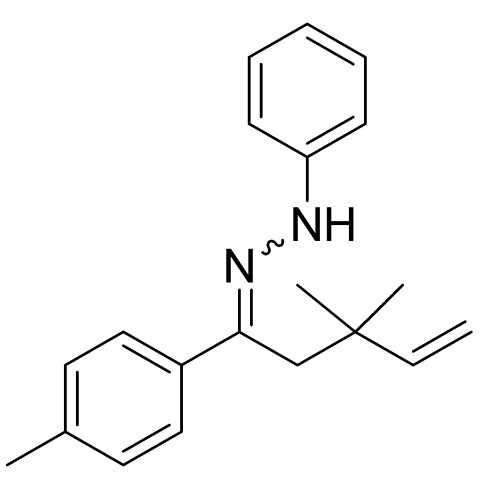

compound (2q)

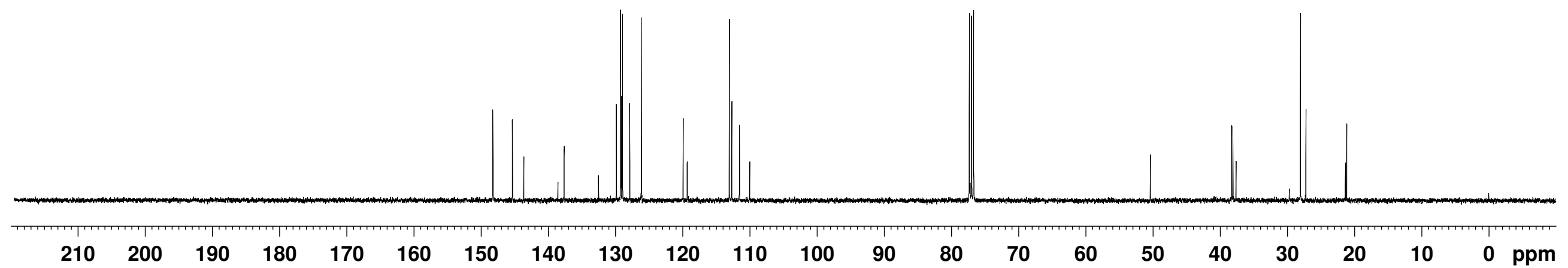




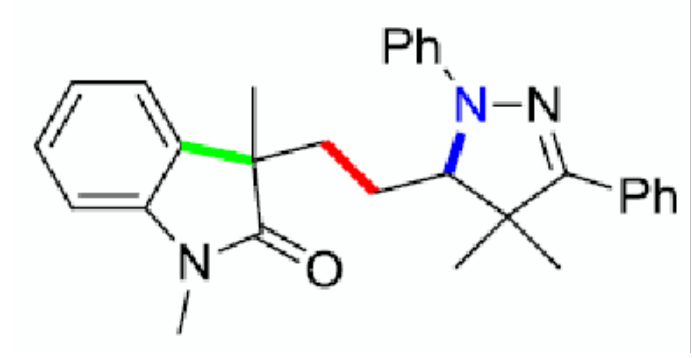

compound (3a)

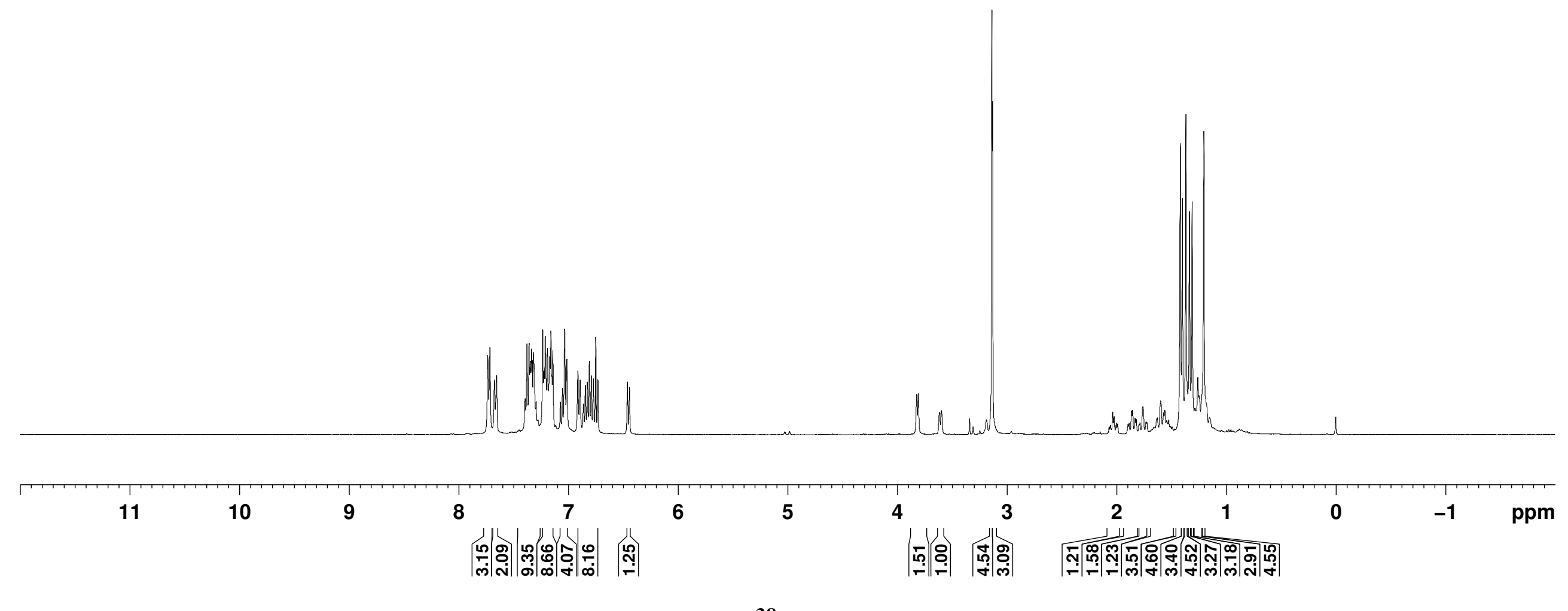



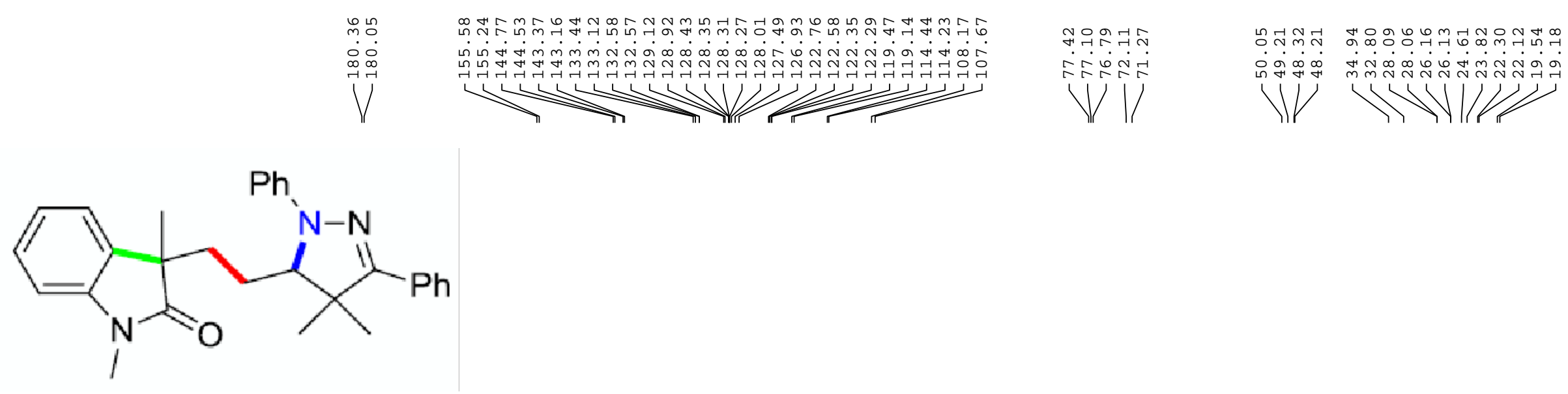

compound (3a)

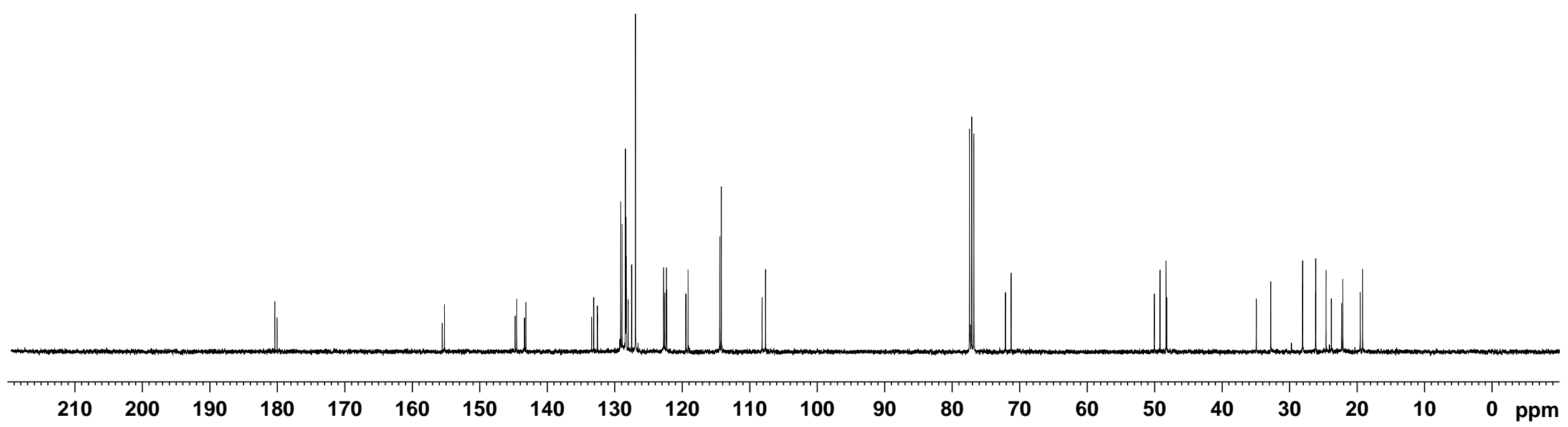




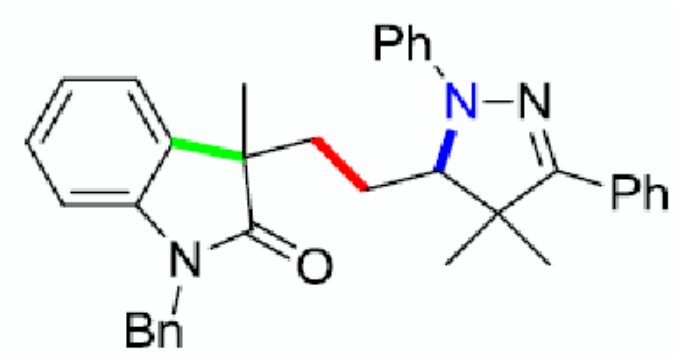

compound (3b)

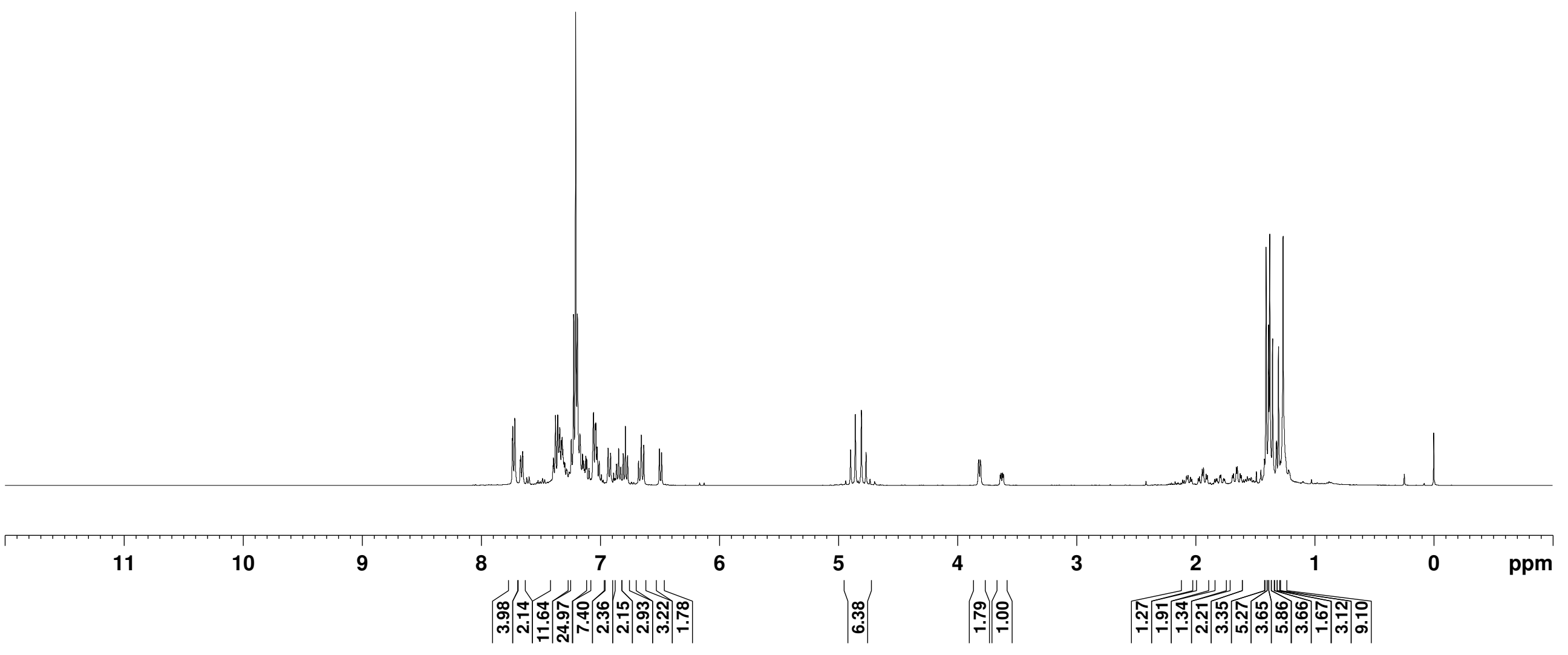




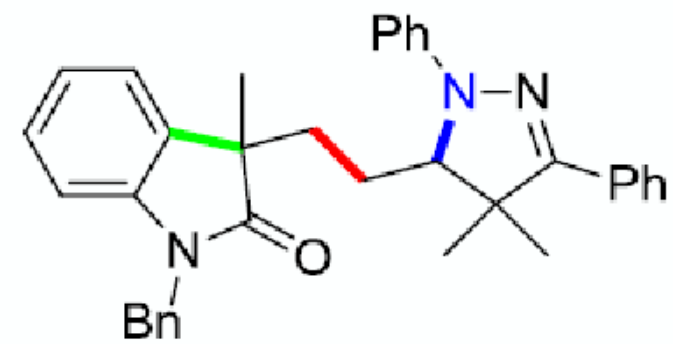

compound (3b)

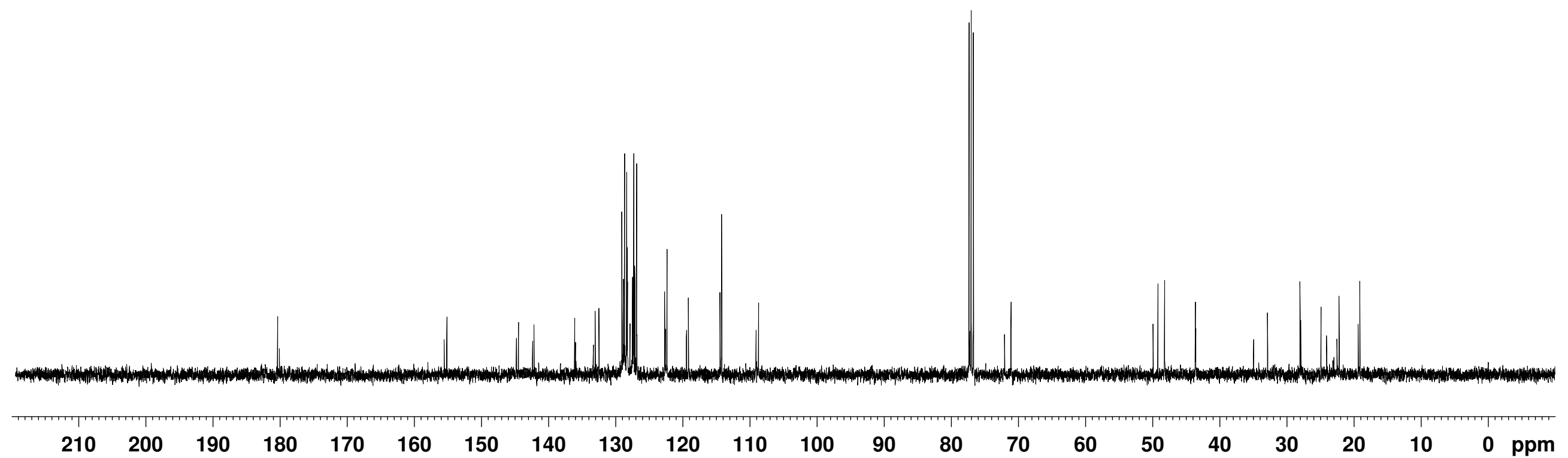




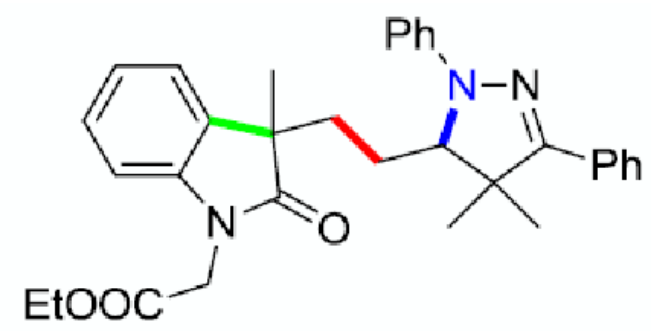

compound (3c)

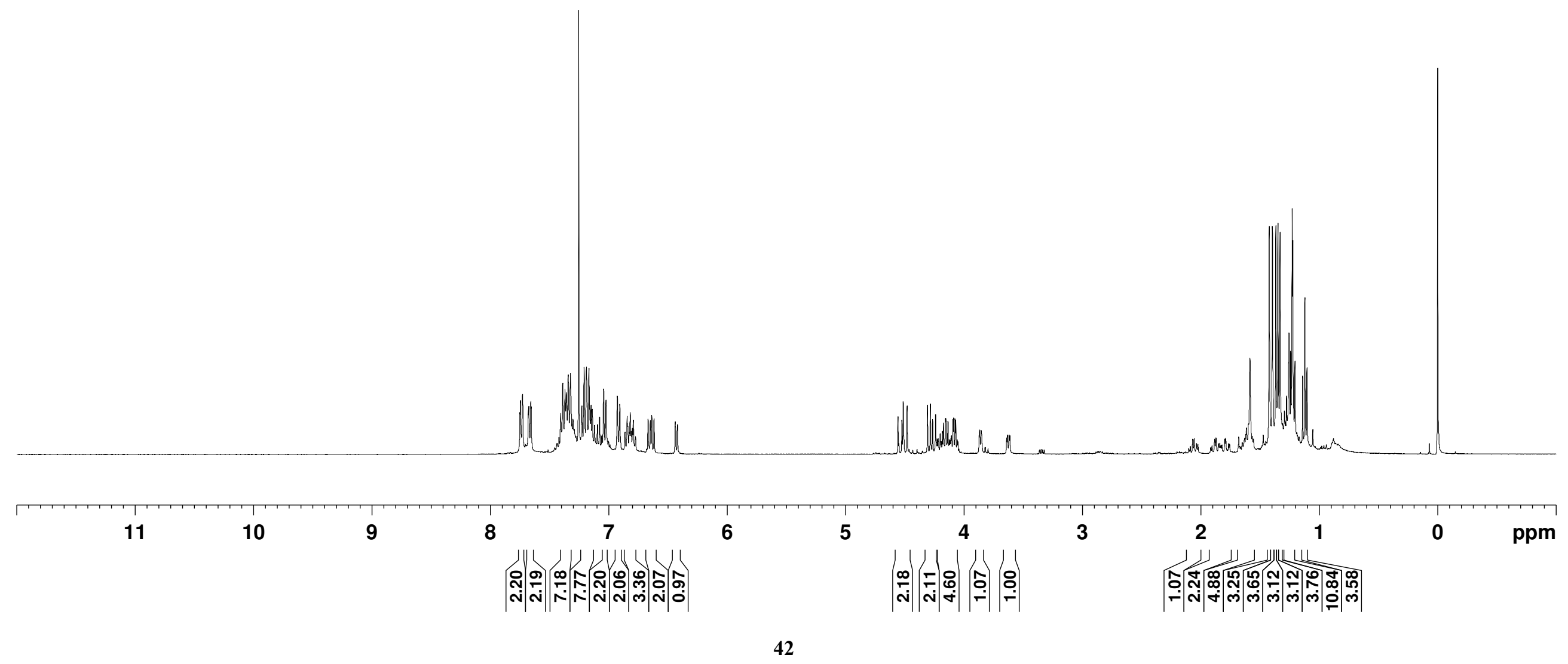



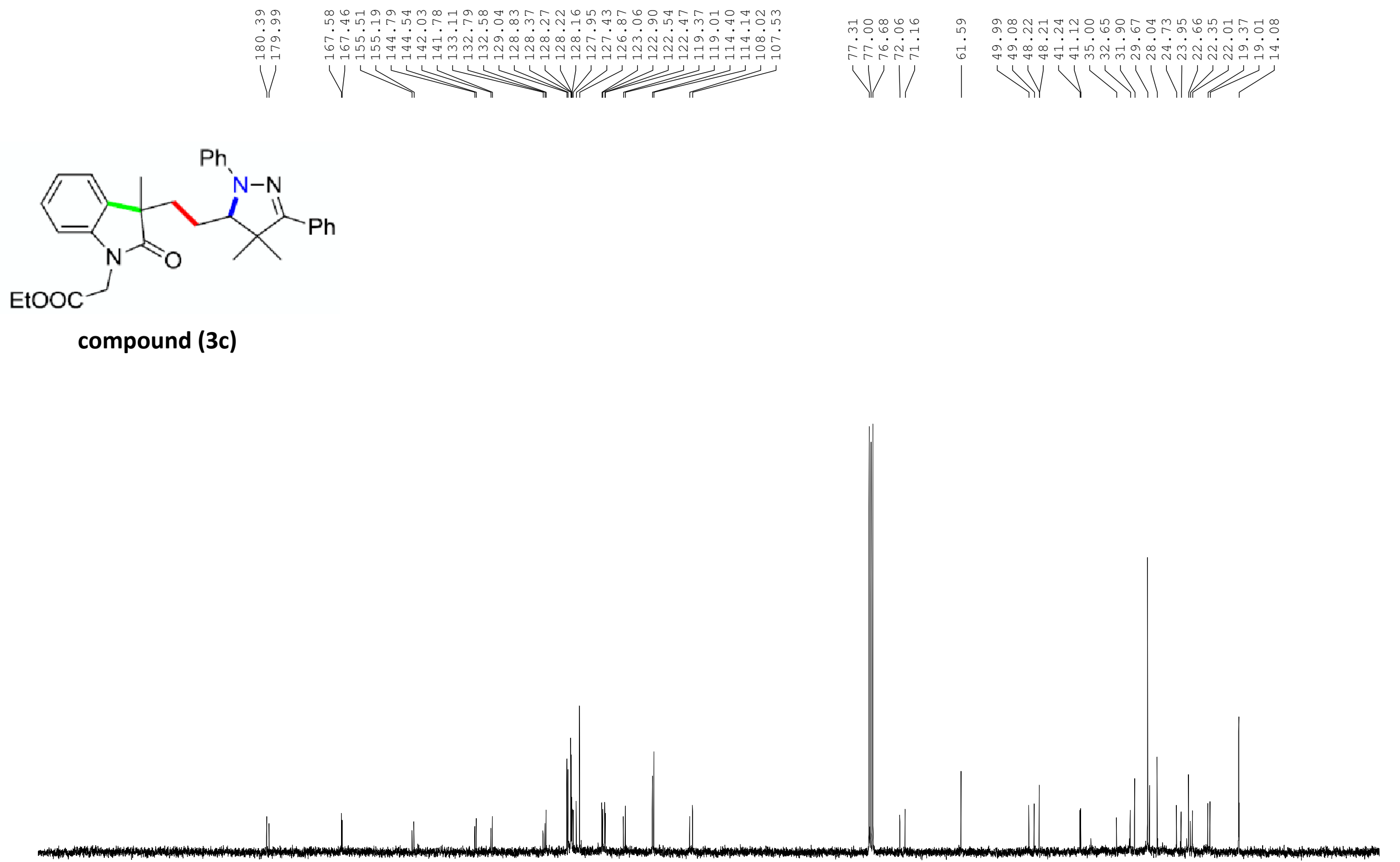

210200

$190 \quad 180$

170

160

150

140

30

120

110 


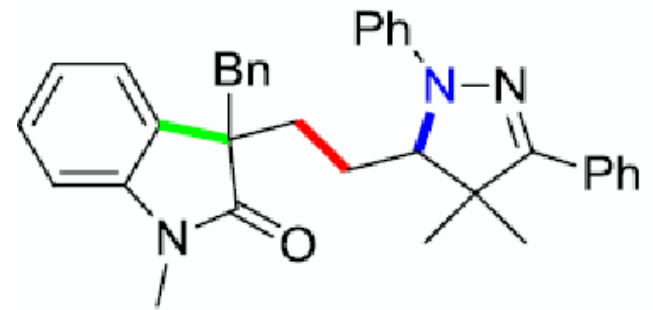

compound (3f)

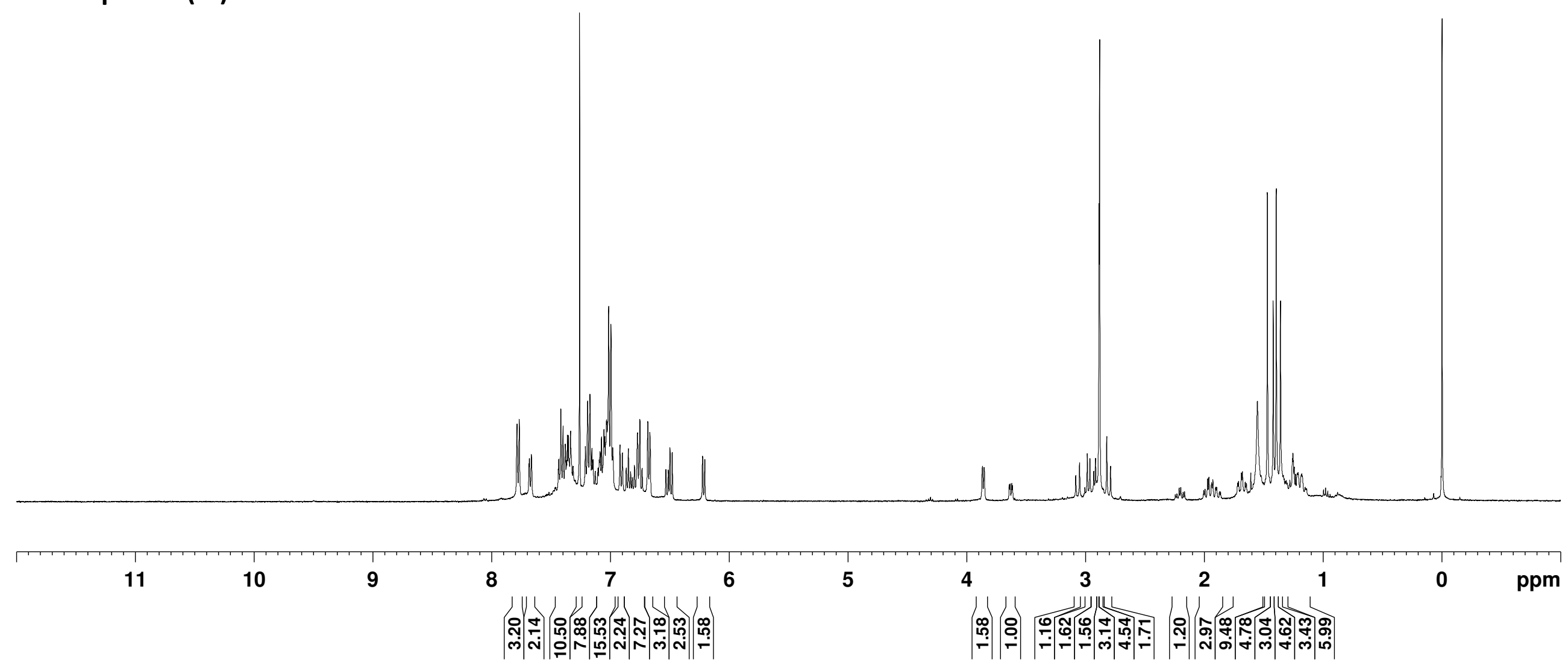




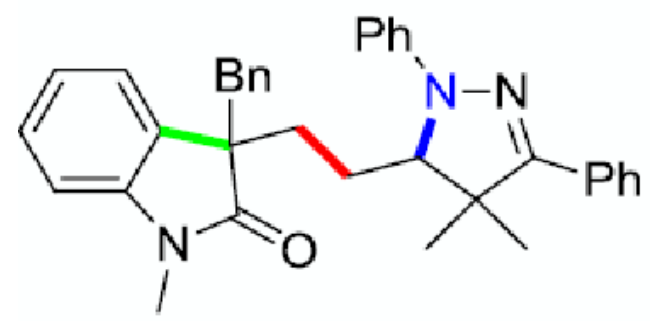

compound (3f)

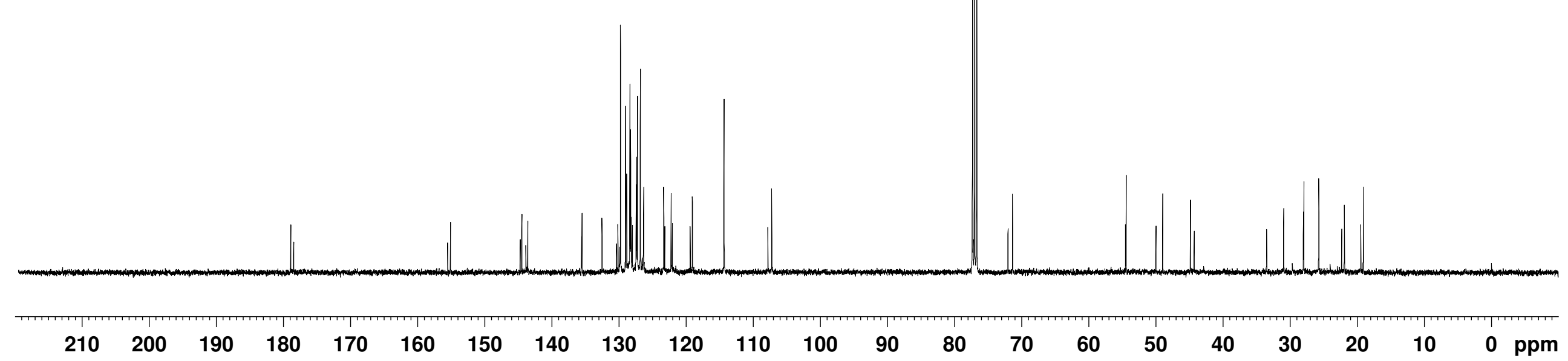



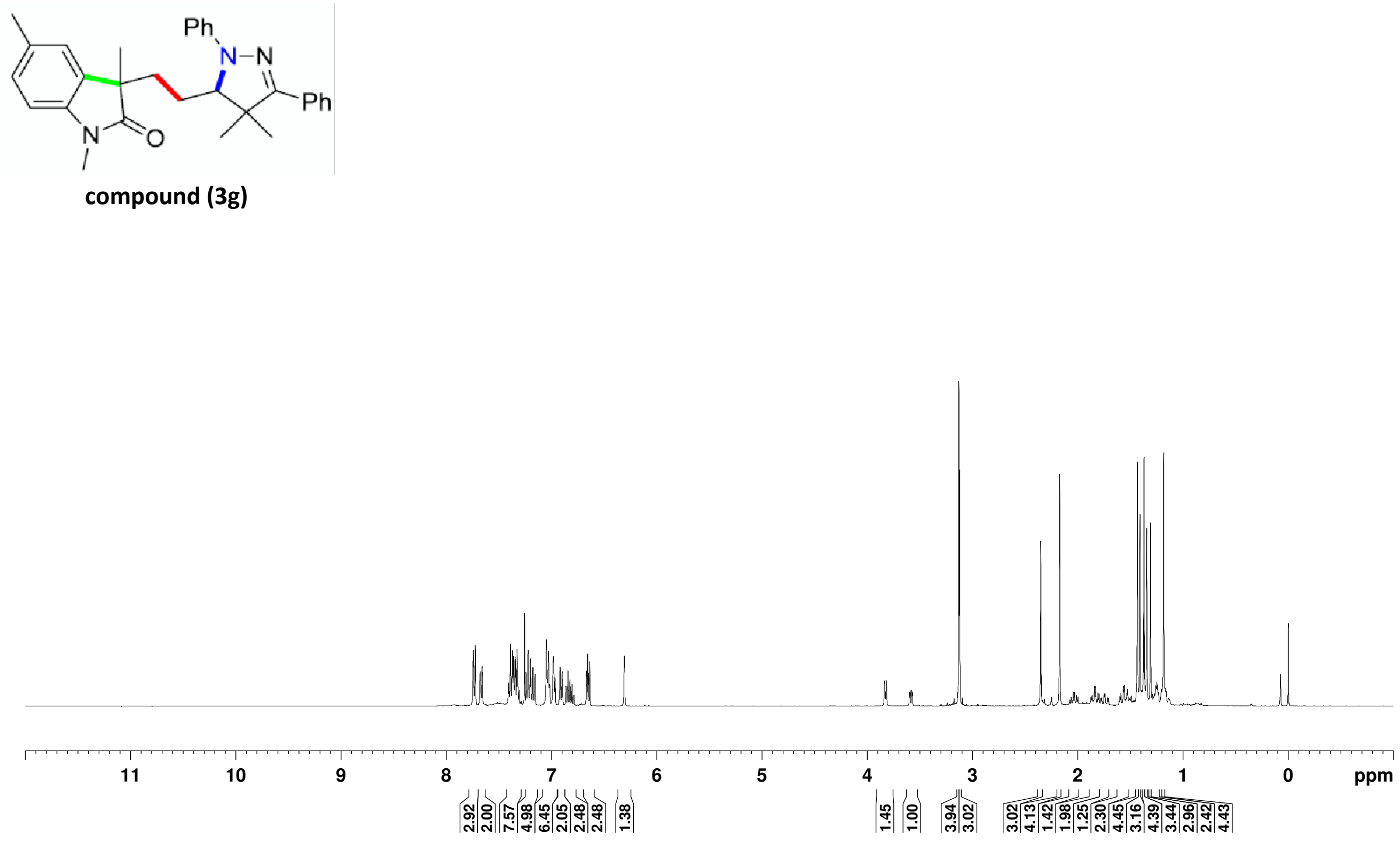

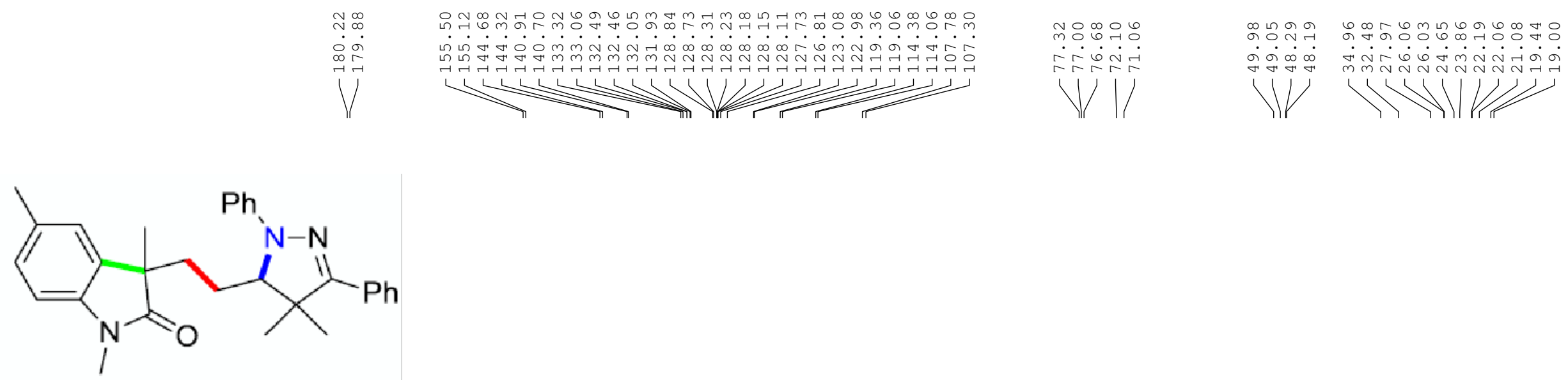

compound (3g)

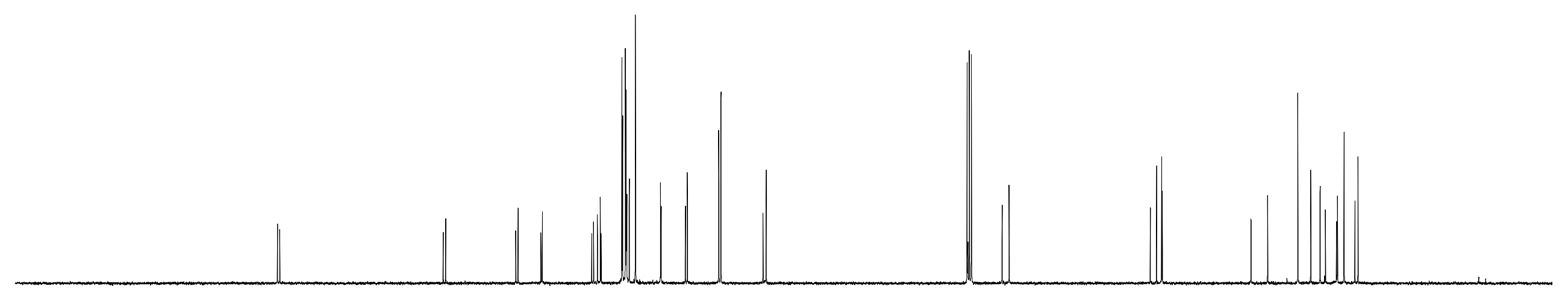

$\begin{array}{lllllllllllllllllllllllllllll}210 & 200 & 190 & 180 & 170 & 160 & 150 & 140 & 130 & 120 & 110 & 100 & 90 & 80 & 70 & 60 & 50 & 40 & 30 & 20 & 10 & 0 & \text { ppm }\end{array}$ 


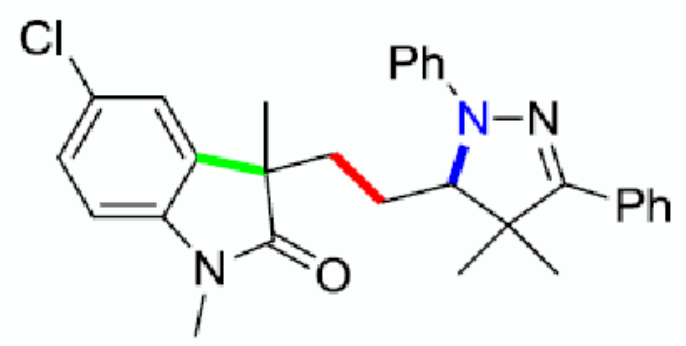

compound (3h)

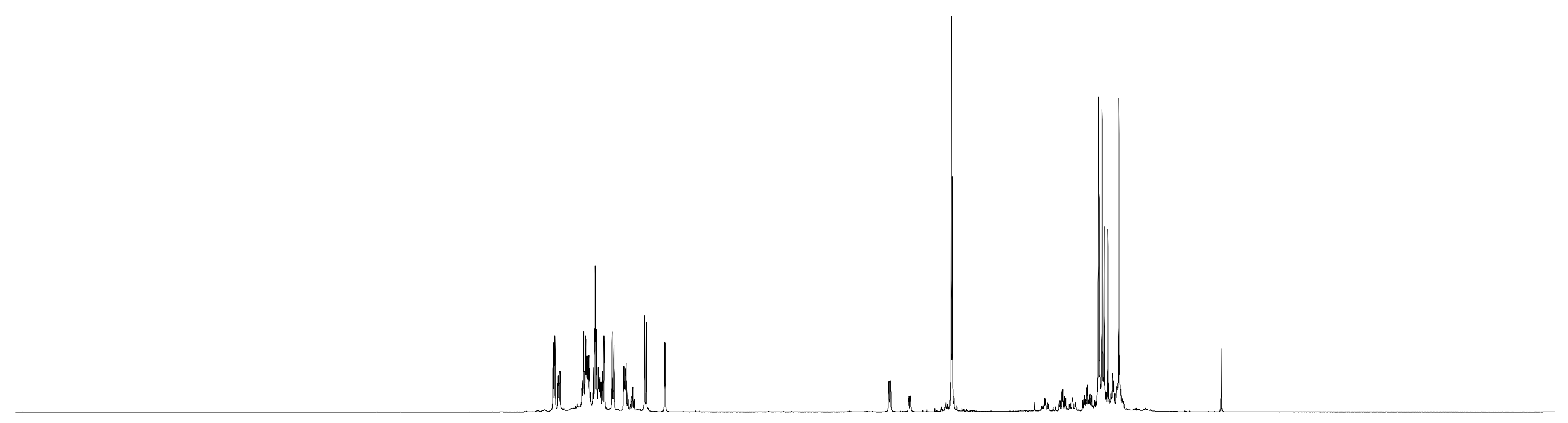

5

4

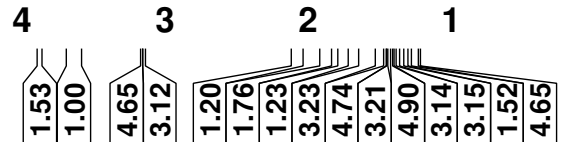



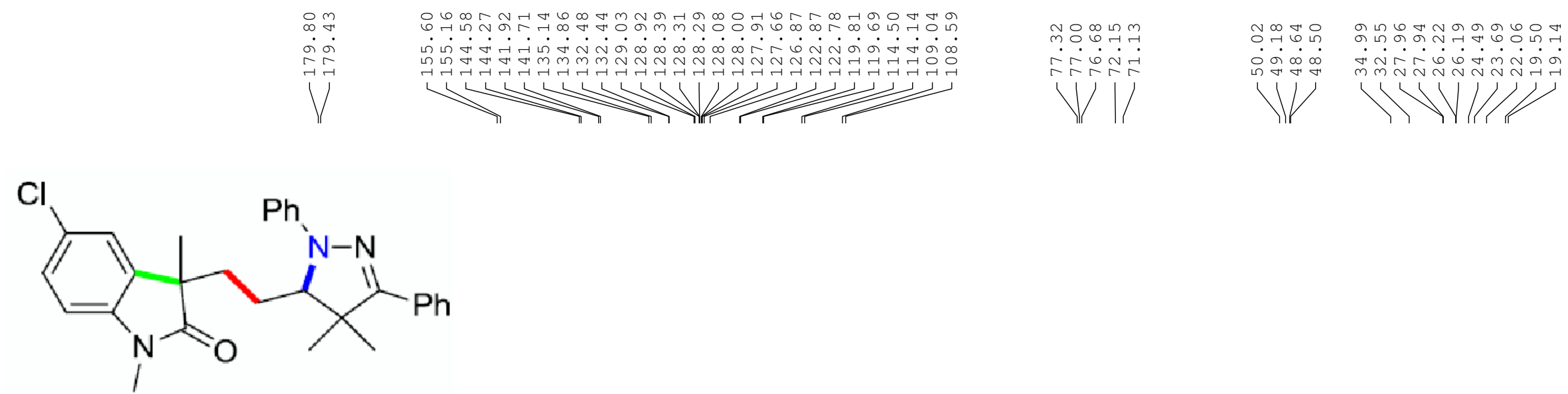

compound (3h)

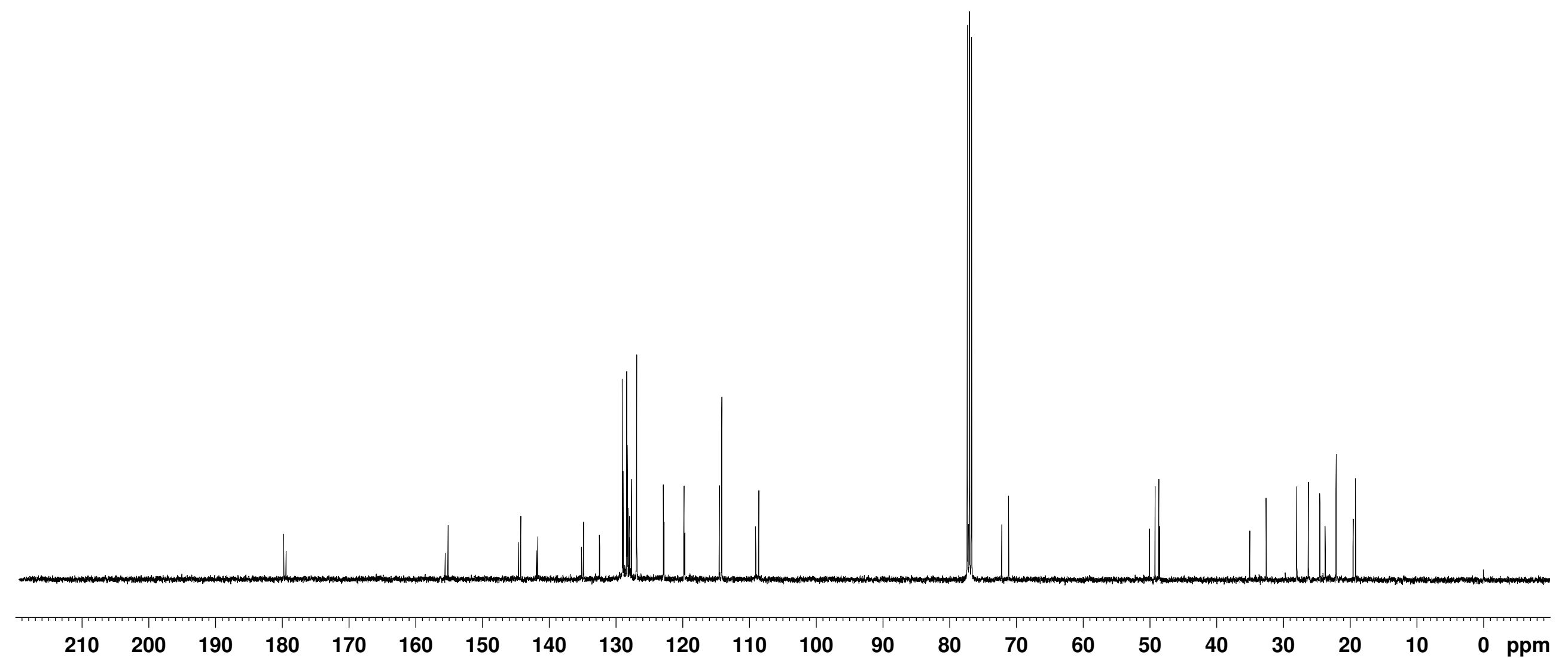




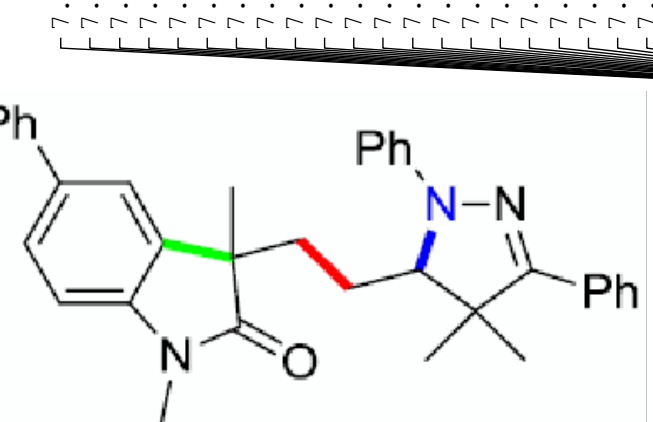

compound (3i)

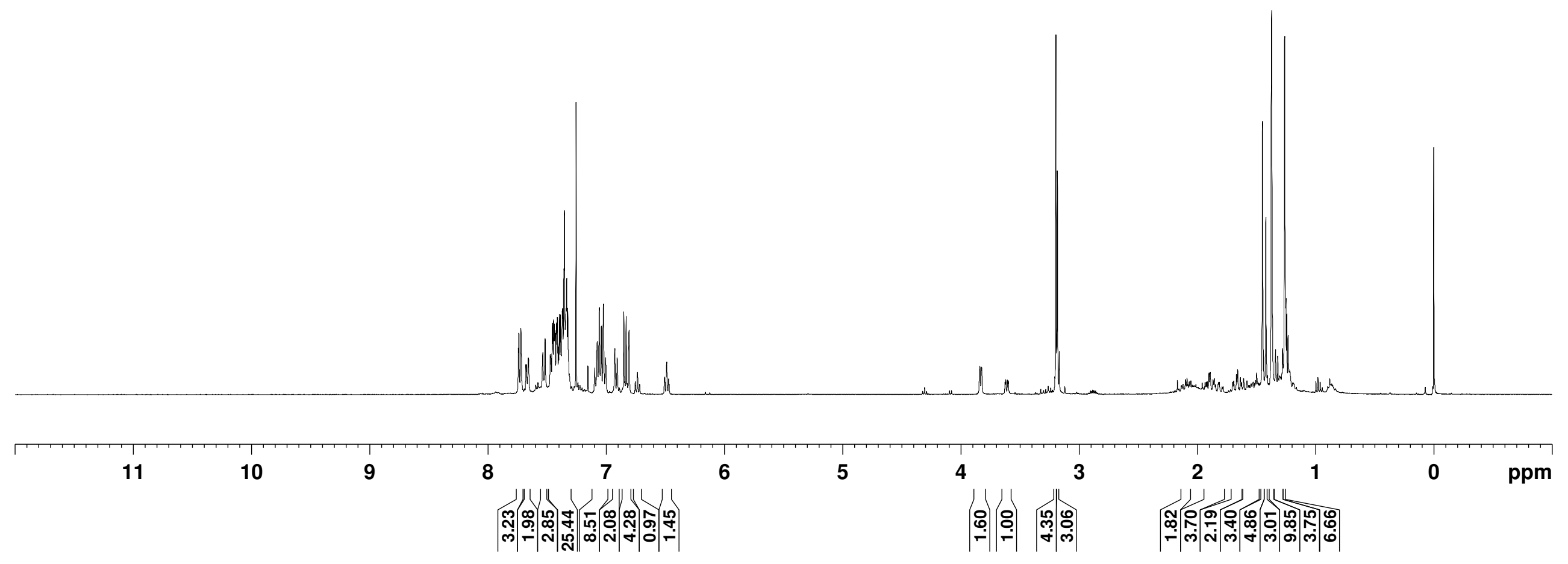



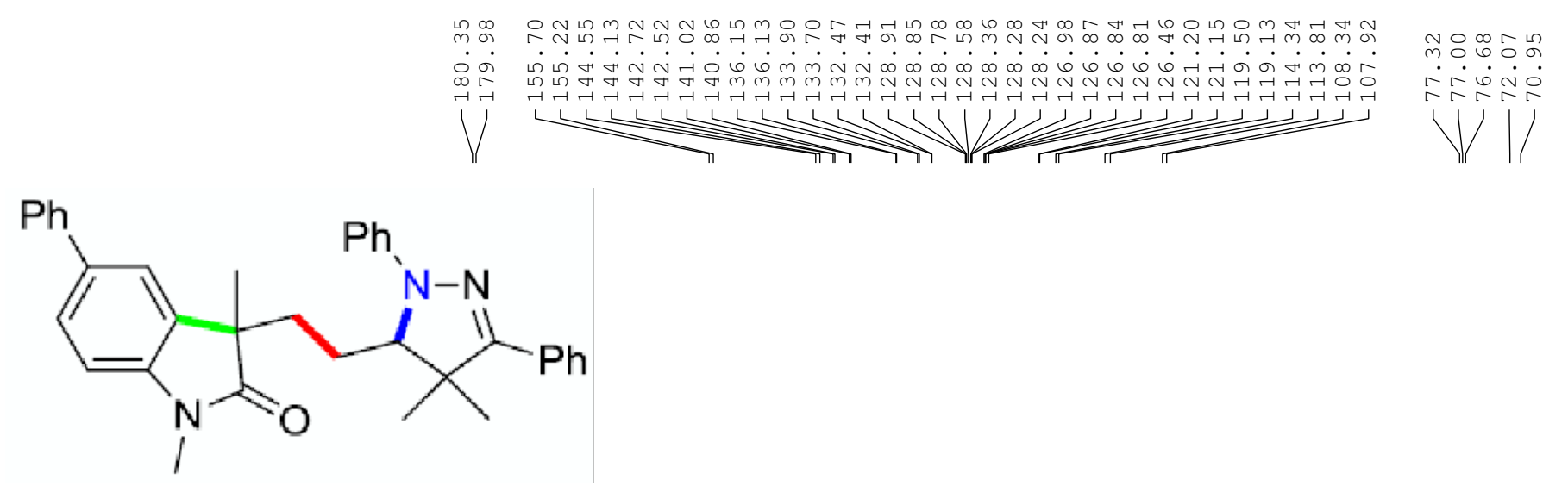

compound (3i)

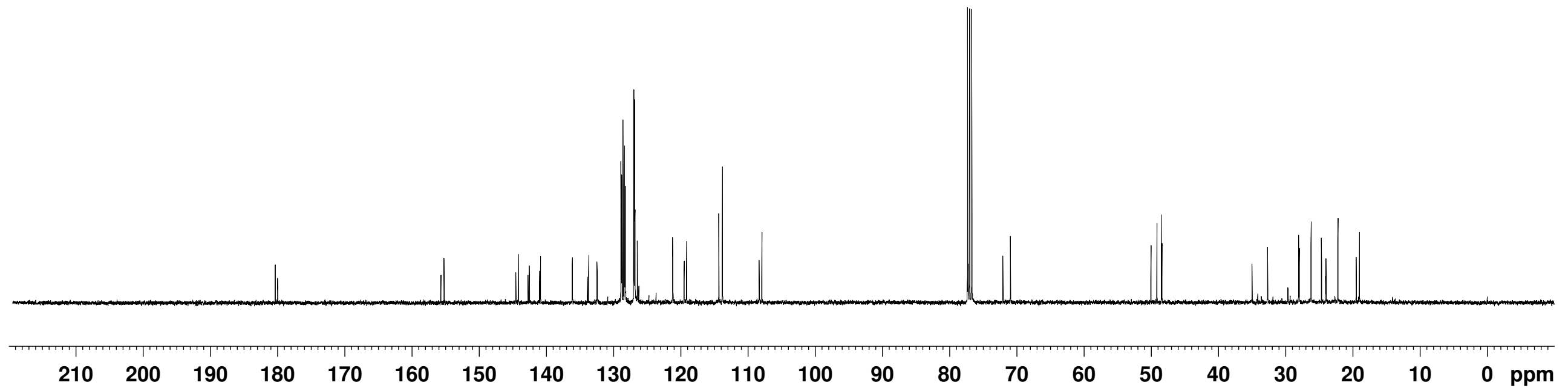



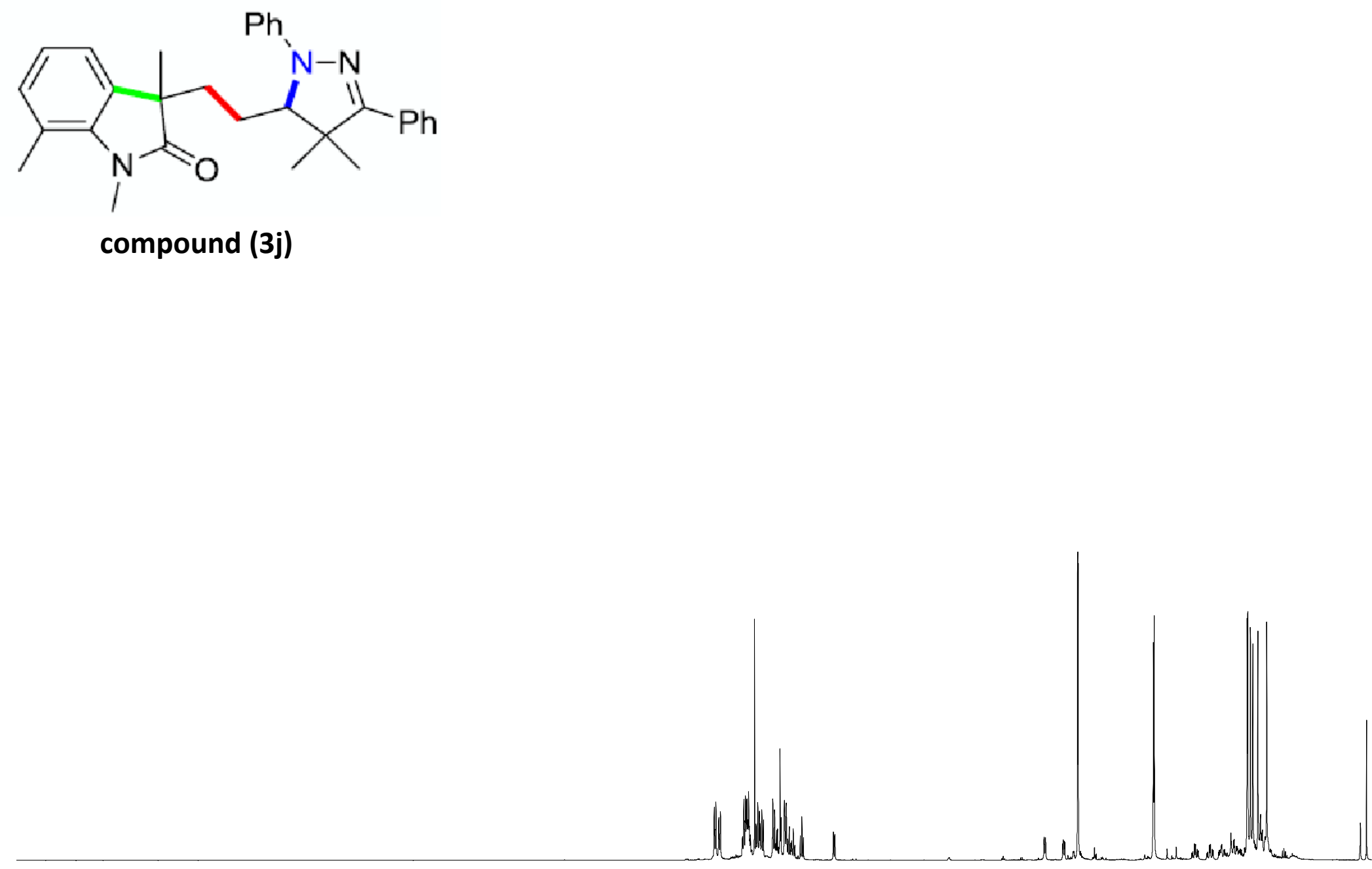


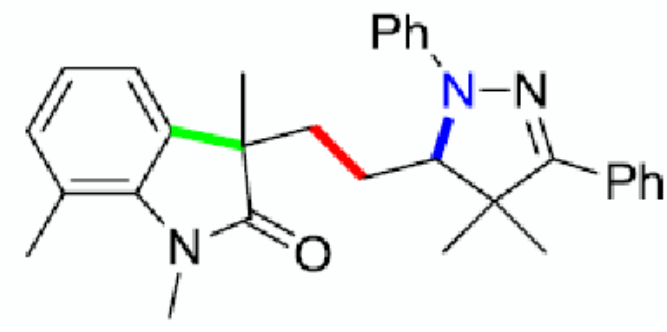

compound (3j)

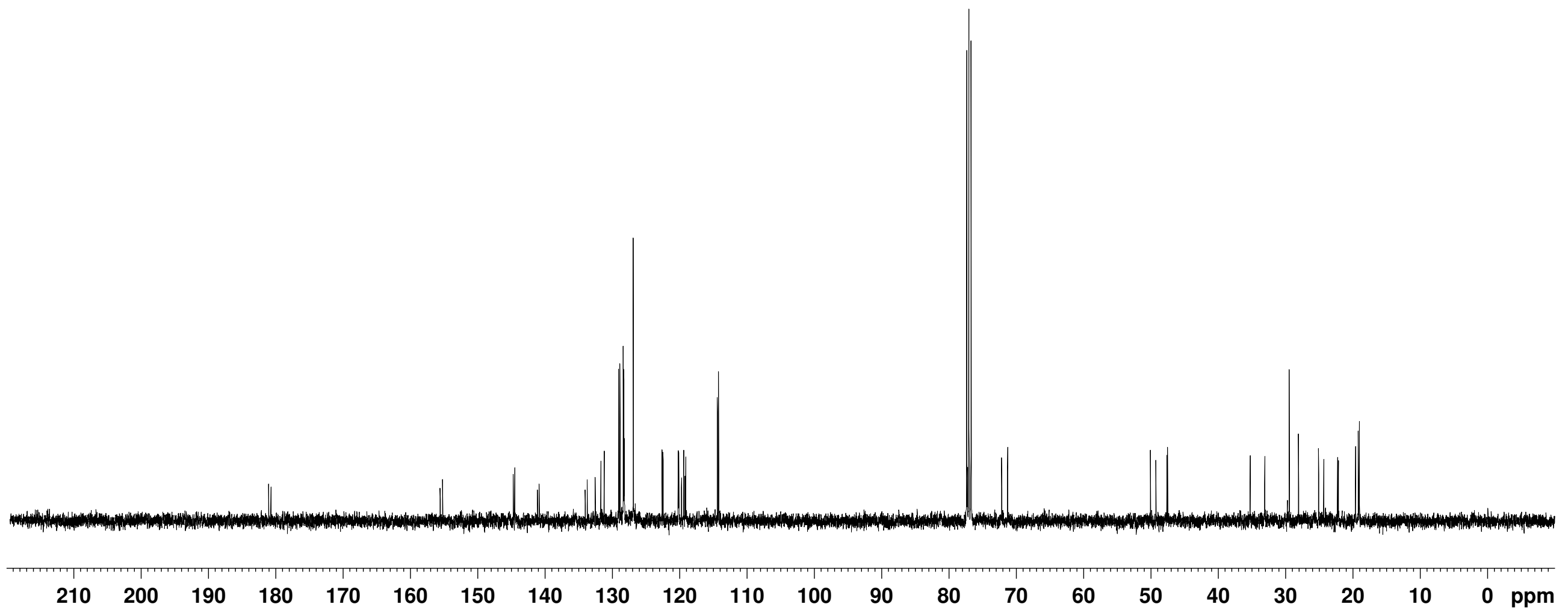




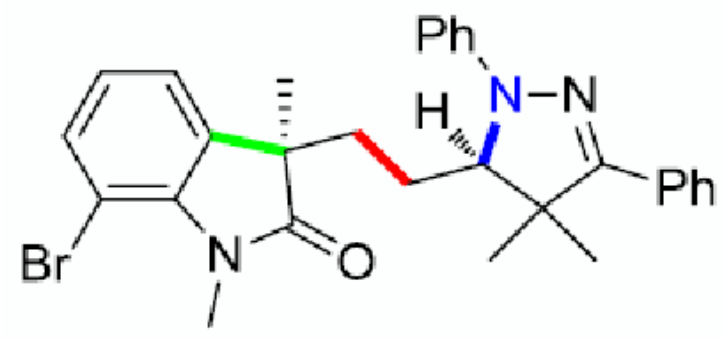

compound (3k)

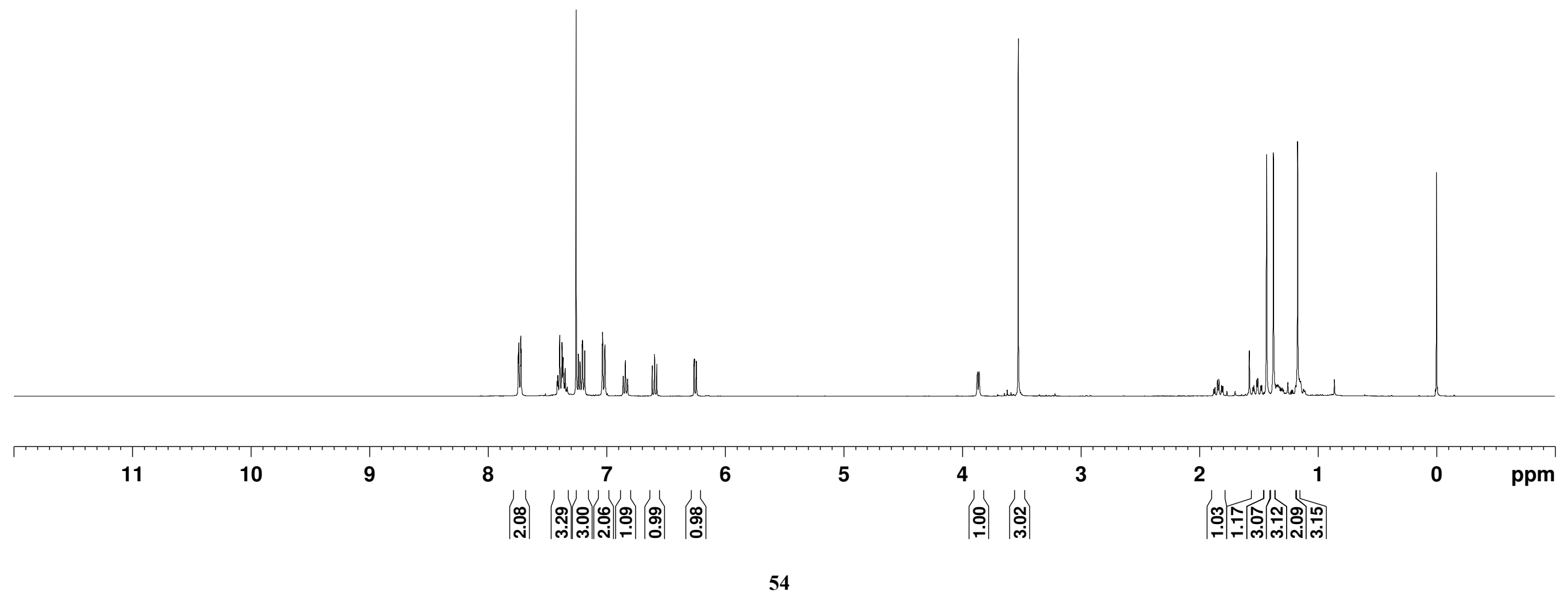



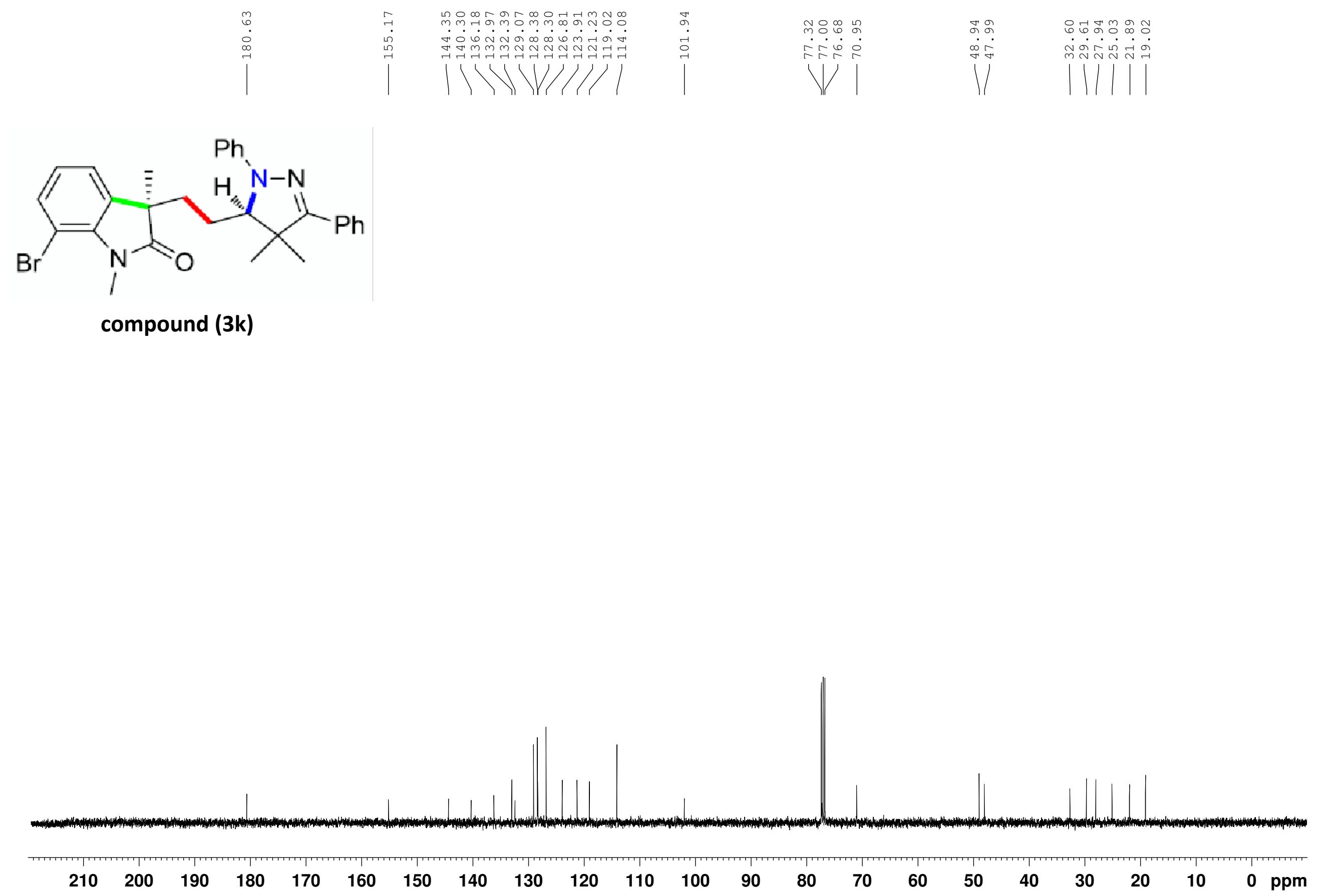


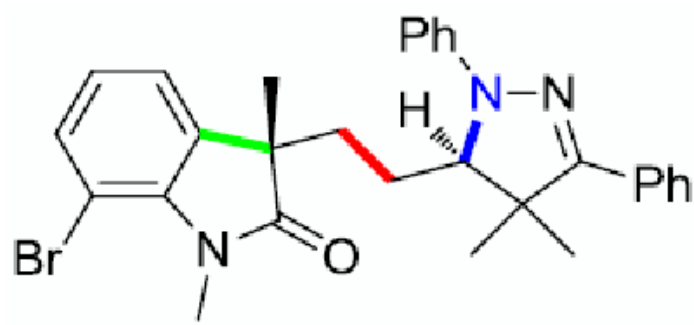

compound (3k')

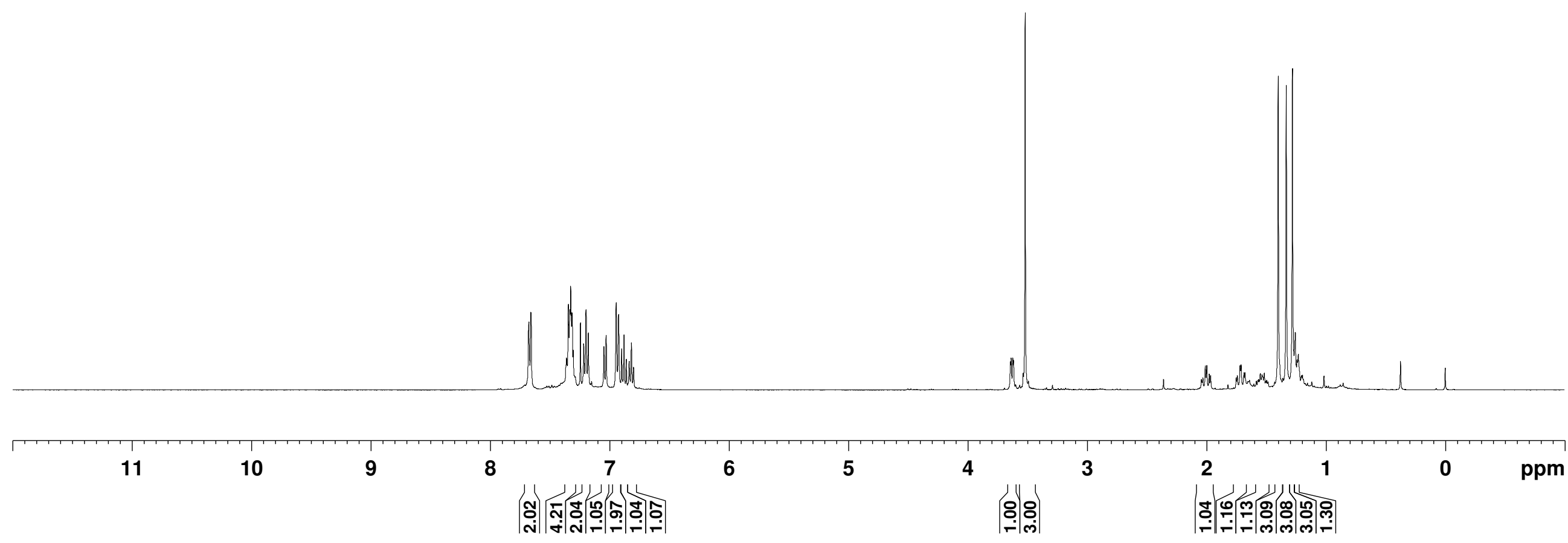




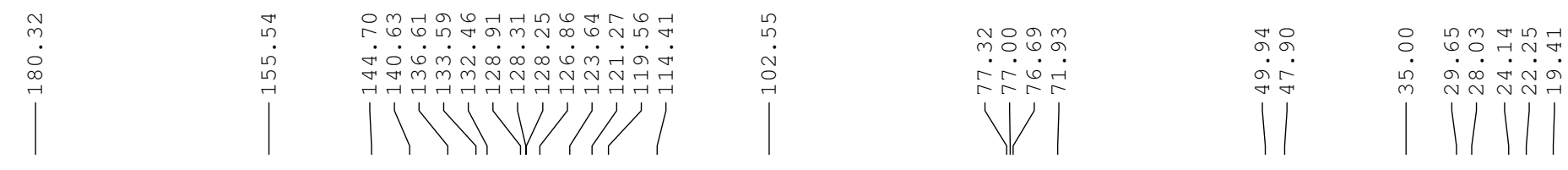

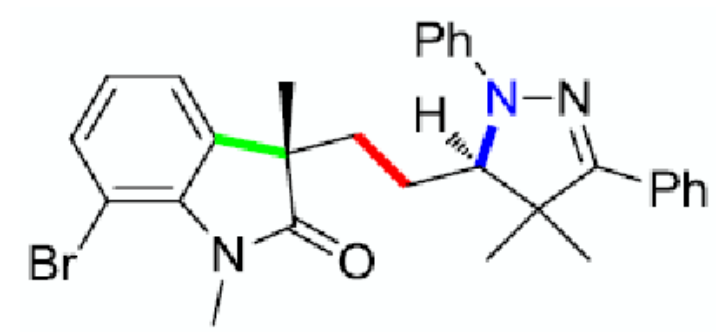

compound (3k')

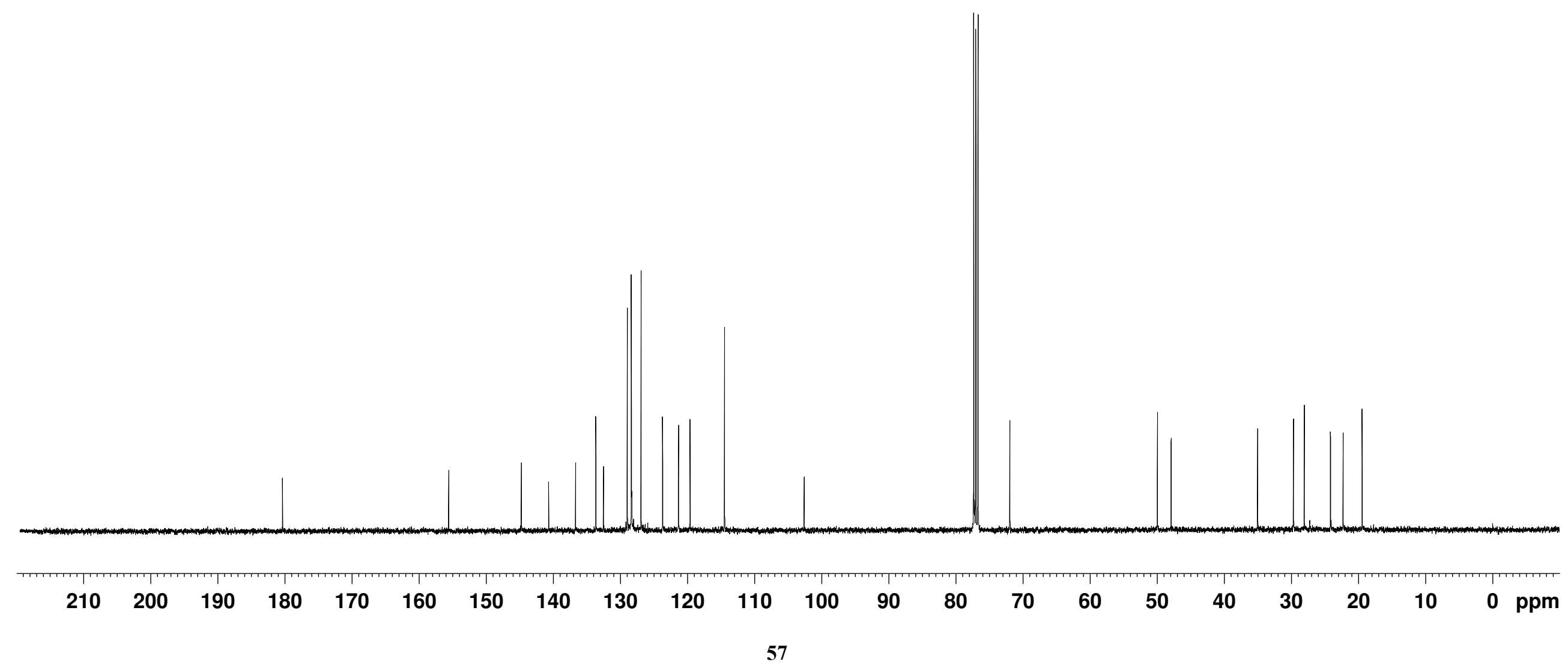




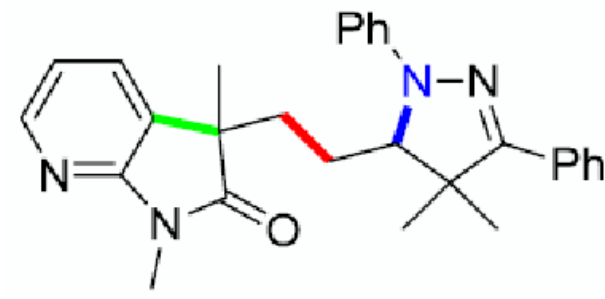

compound (3I)
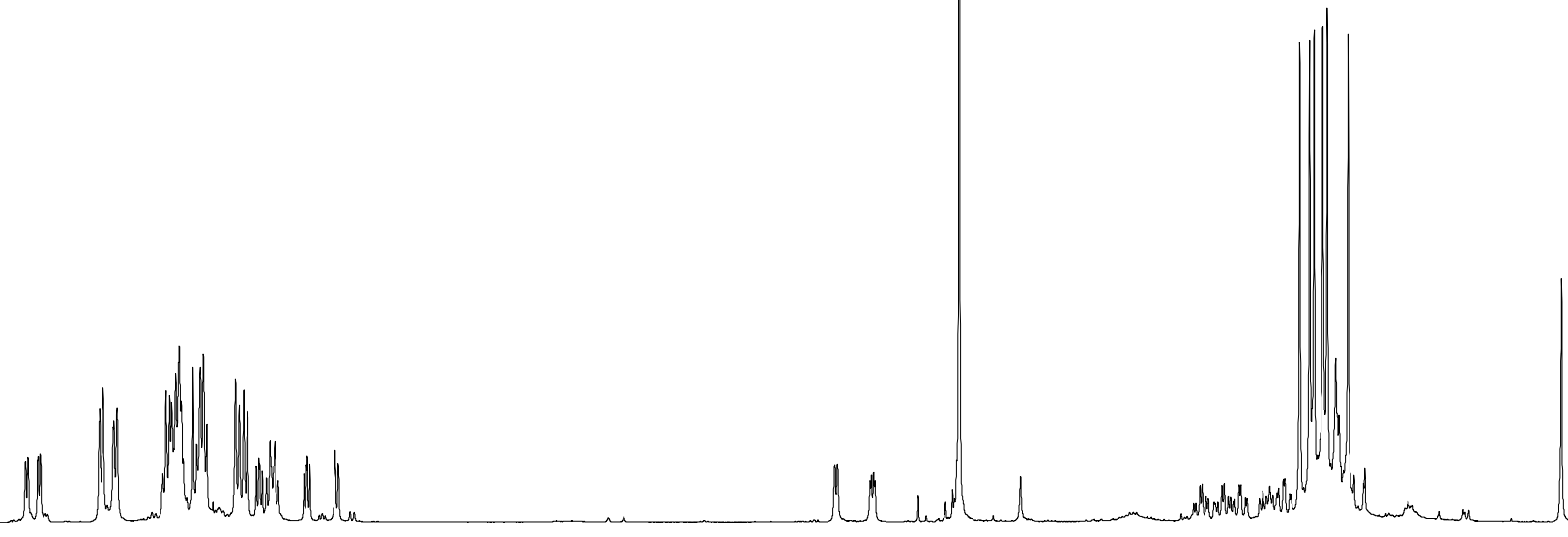

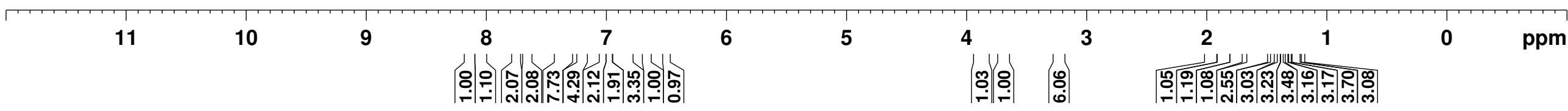



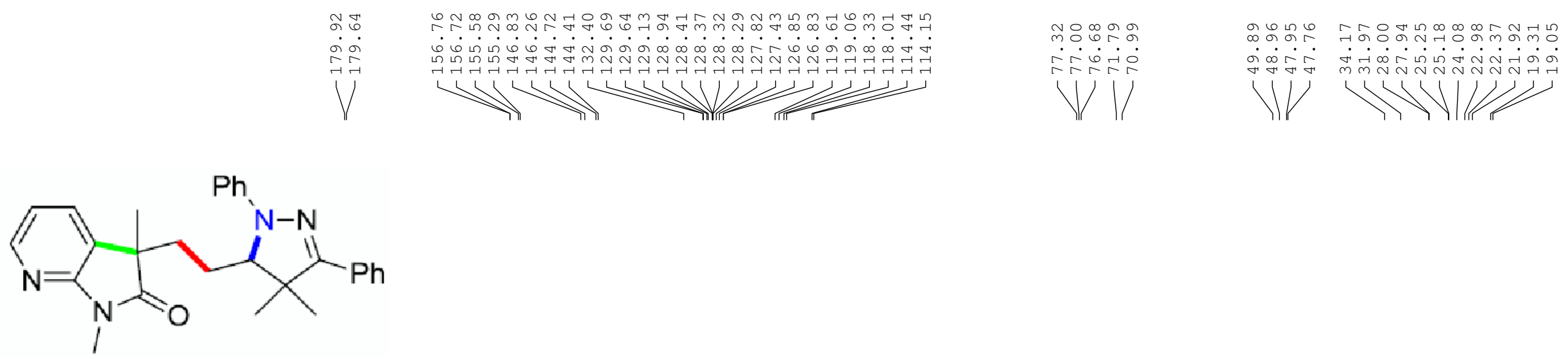

compound (3I)

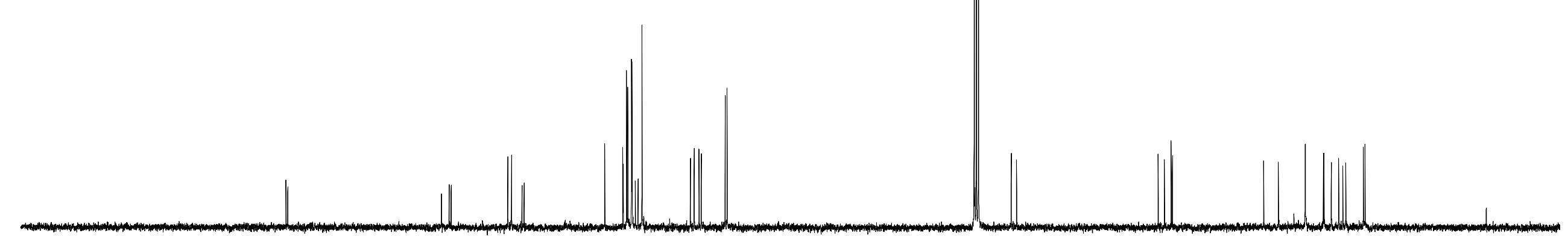




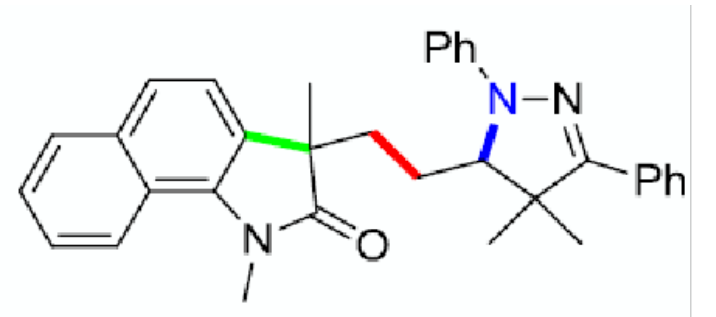

compound (3m)

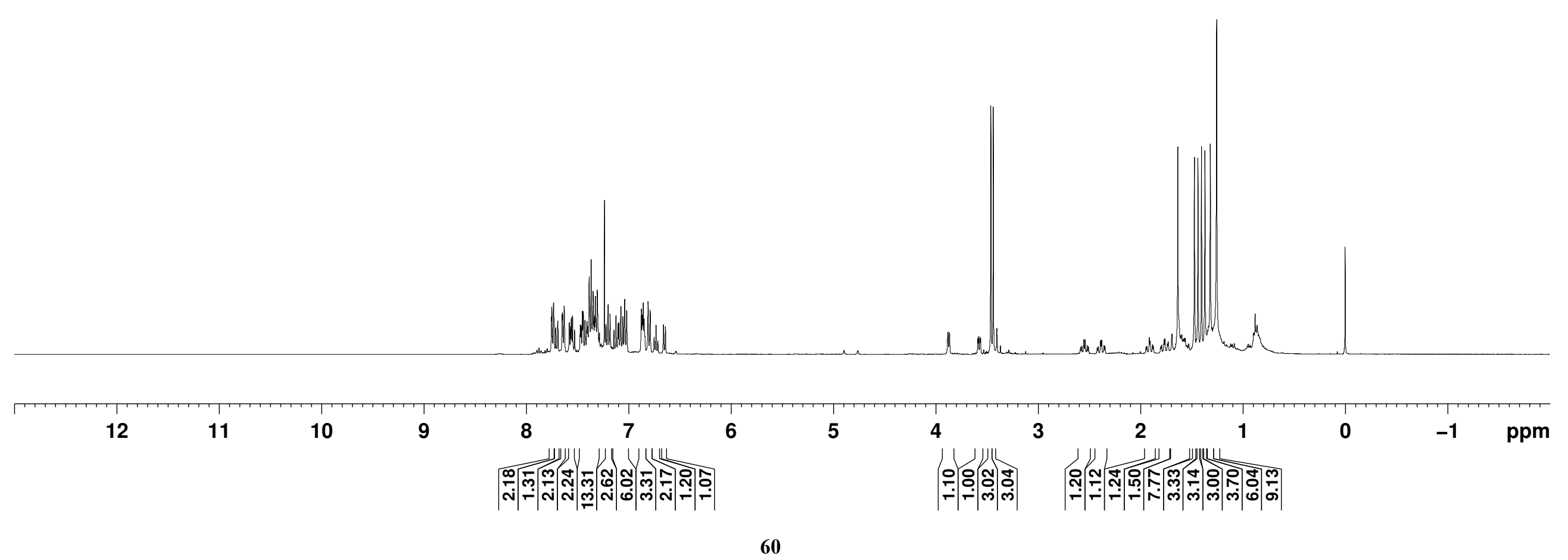




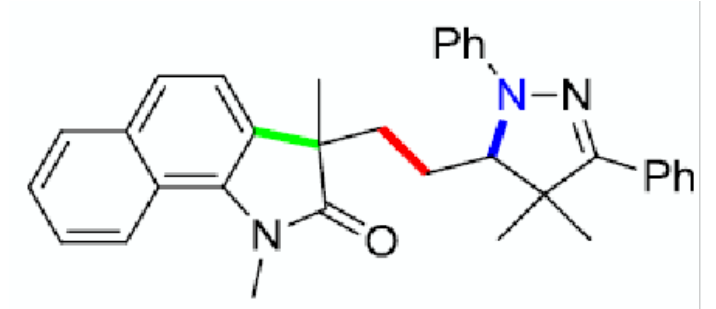

compound (3m)

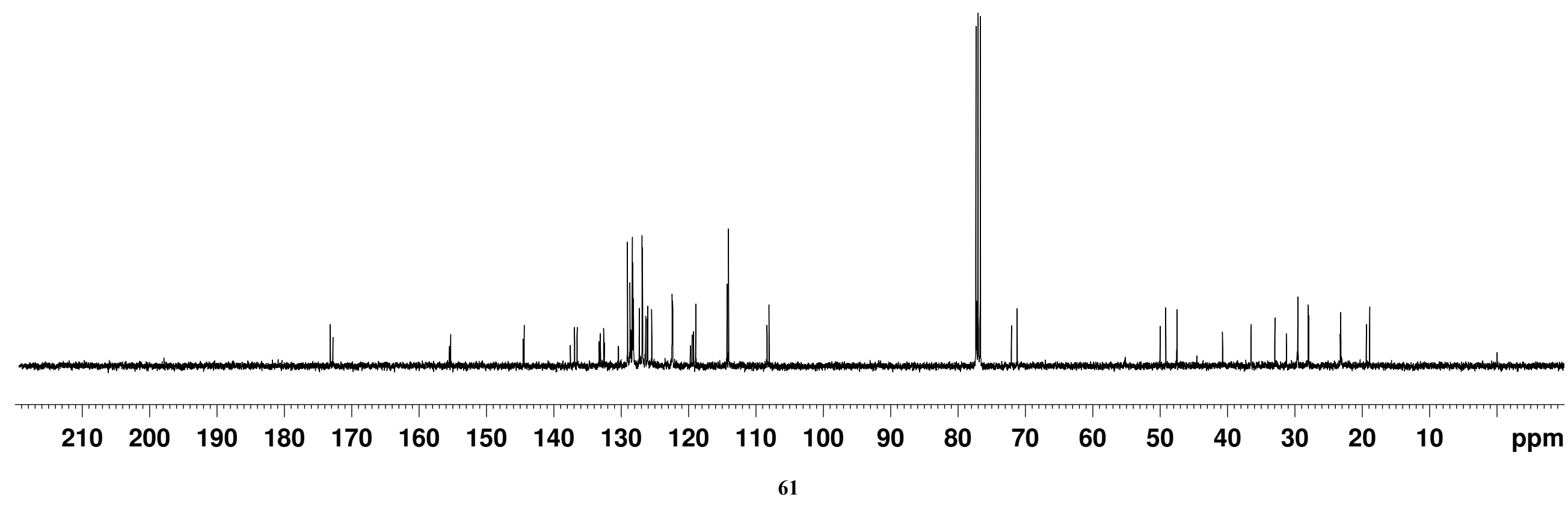




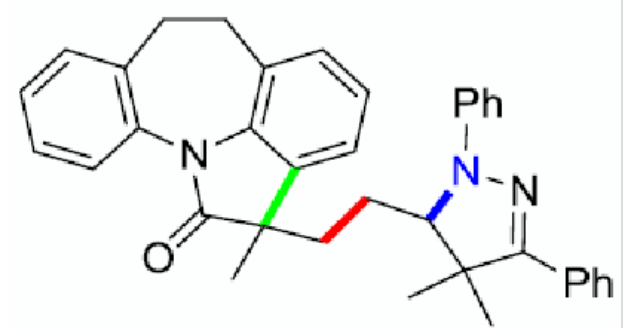

\section{compound (3n)}

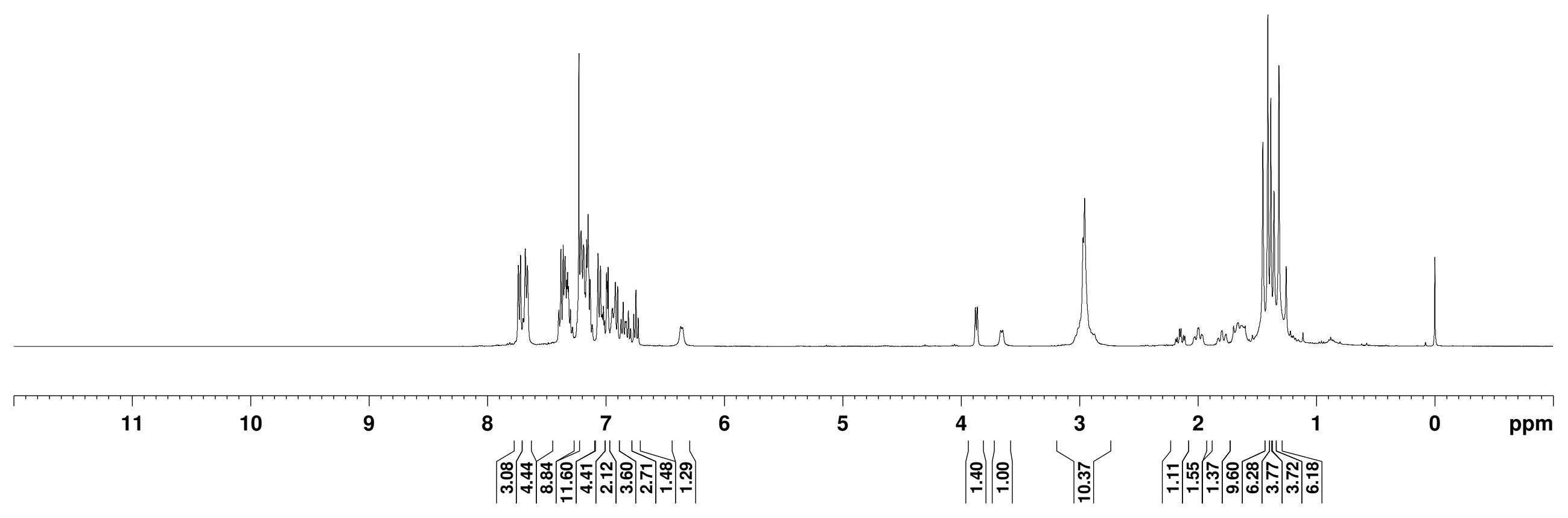




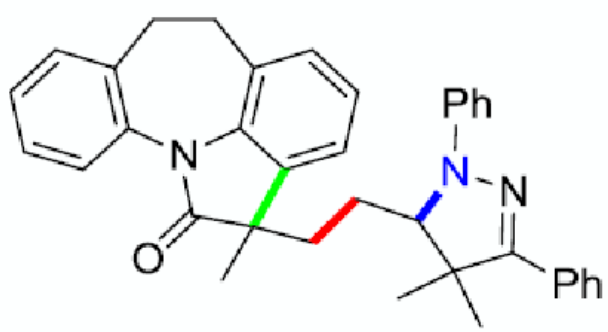

compound (3n)

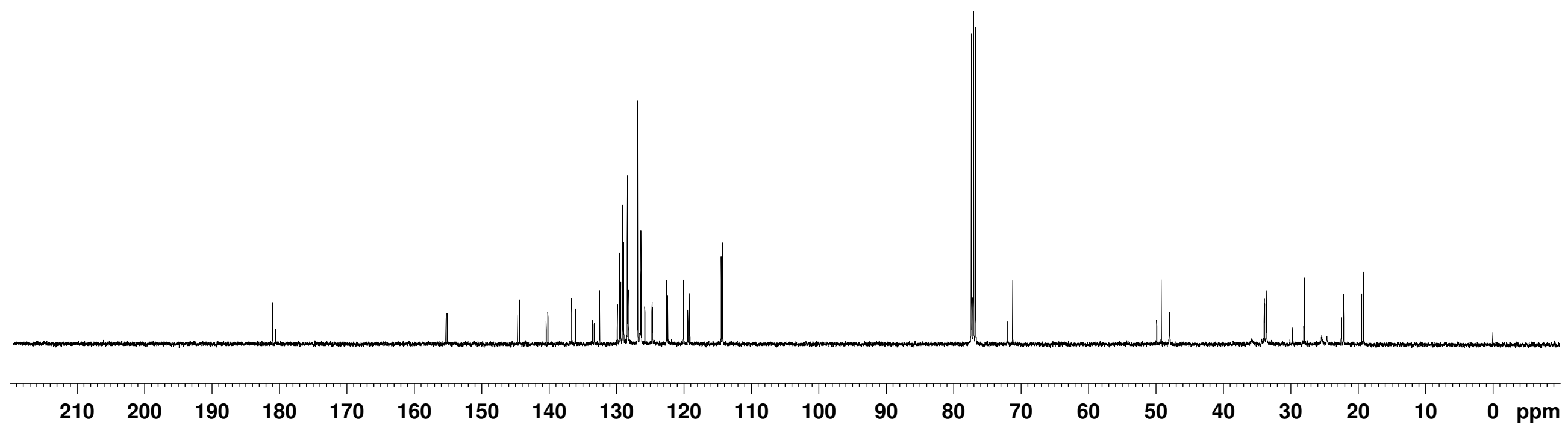



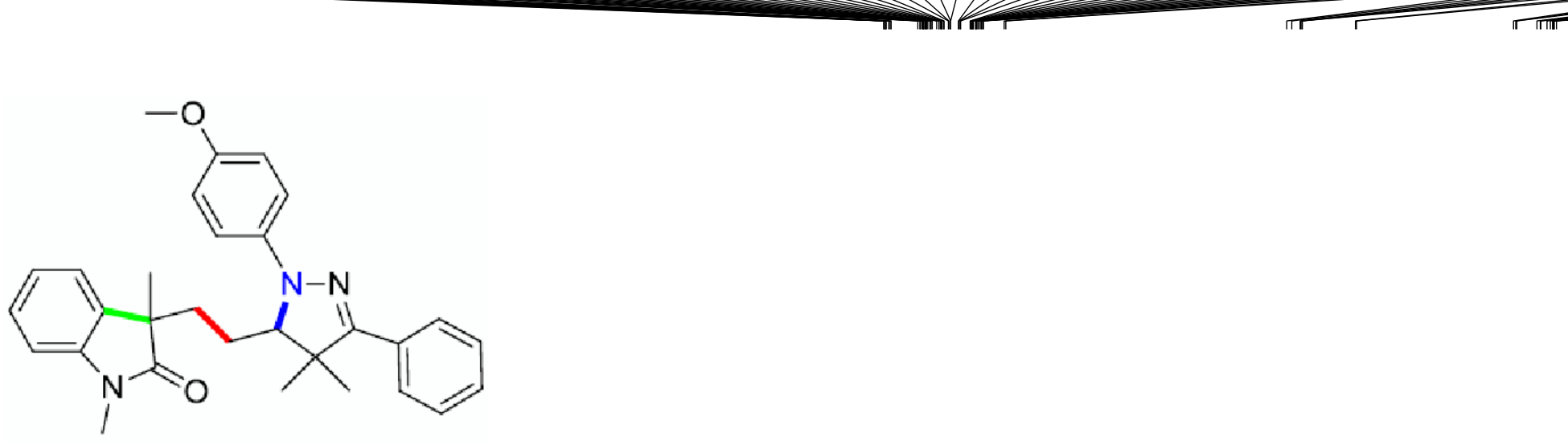

compound (3o)

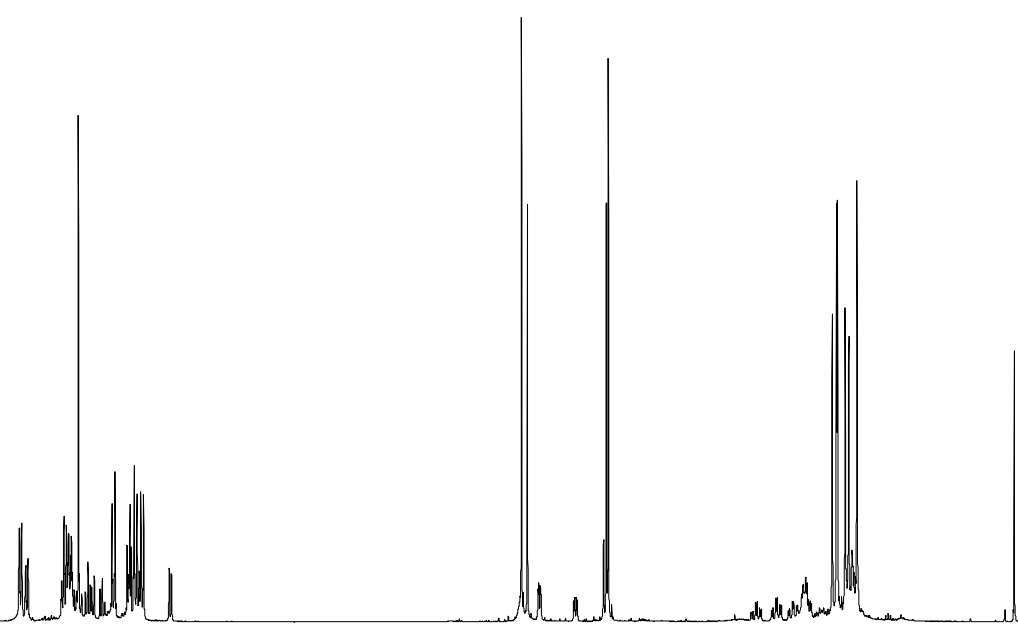




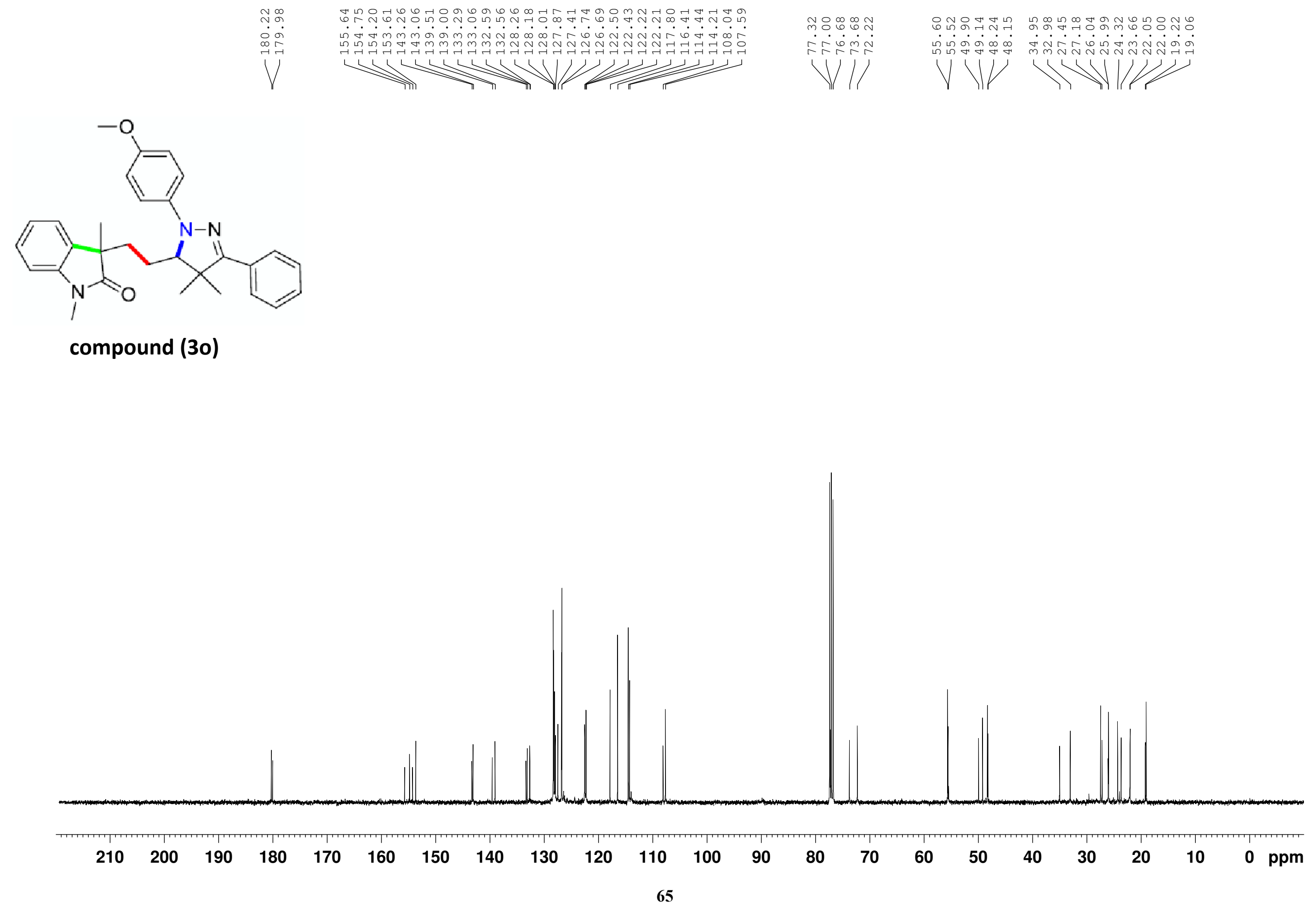




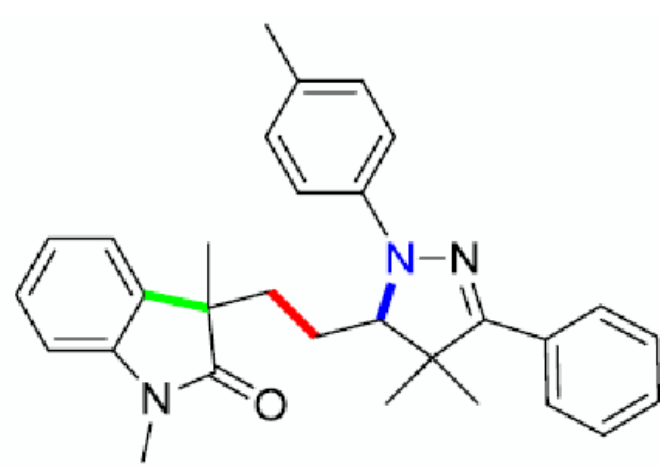

compound (3p)

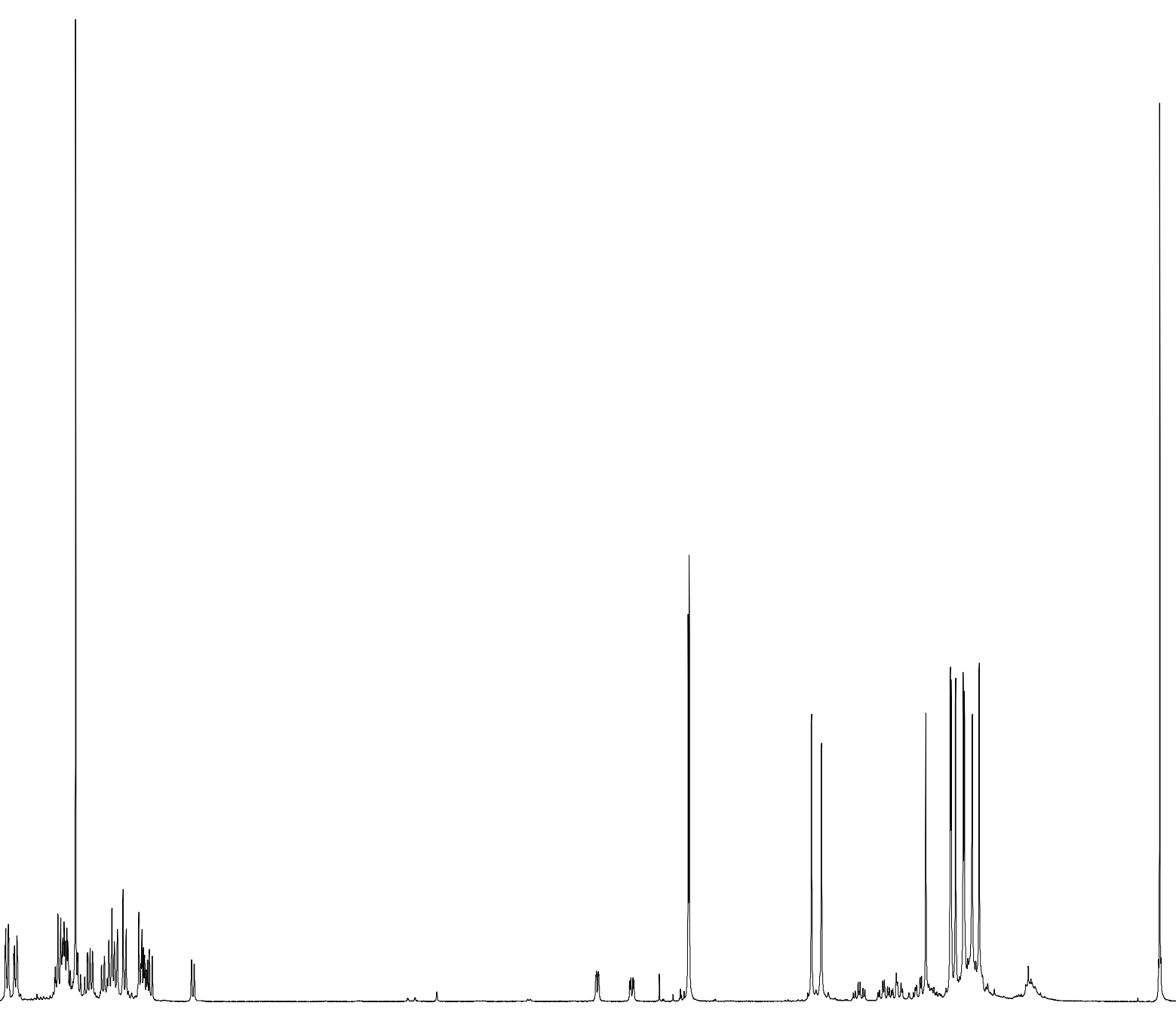
ஸึ่ 

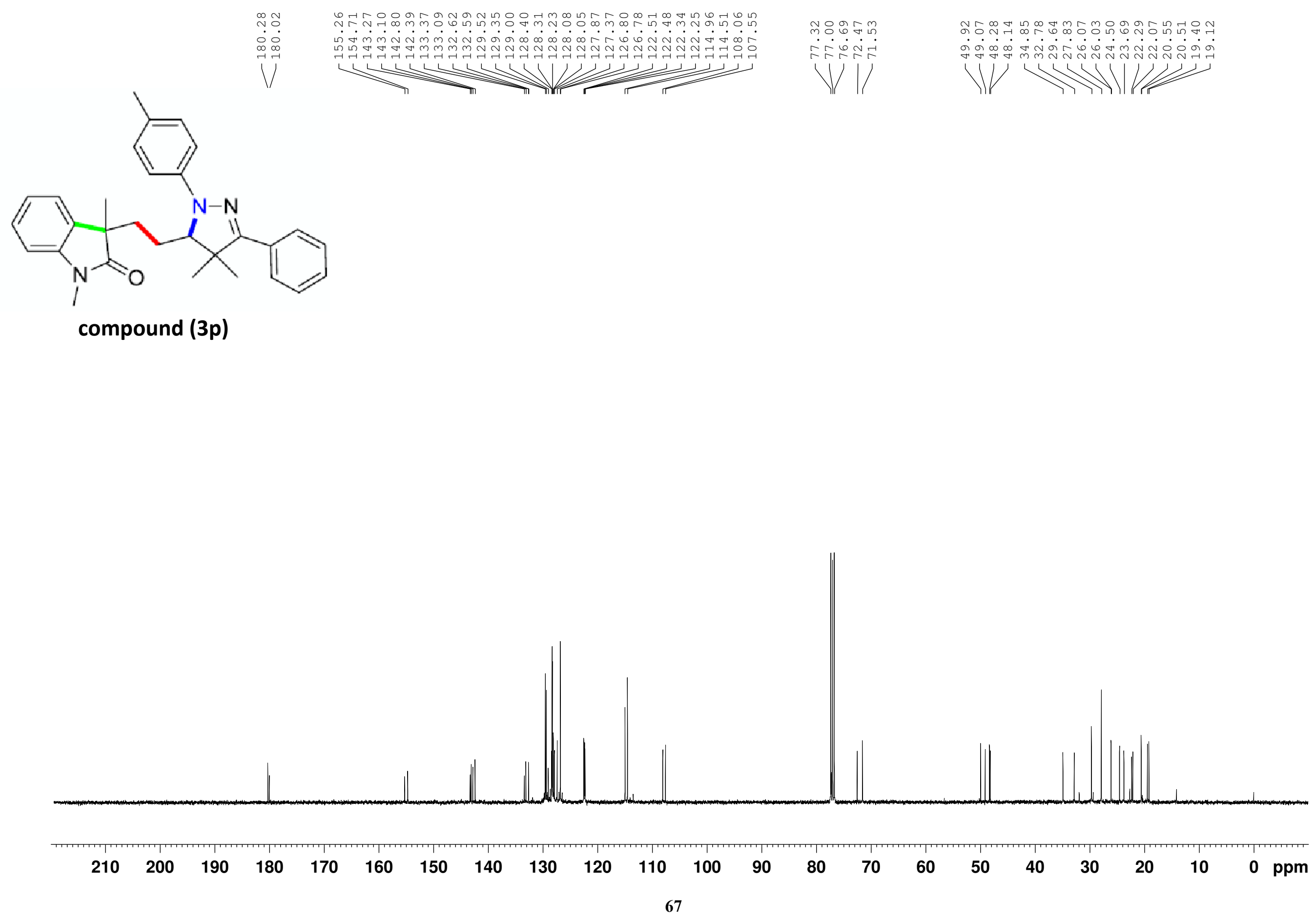

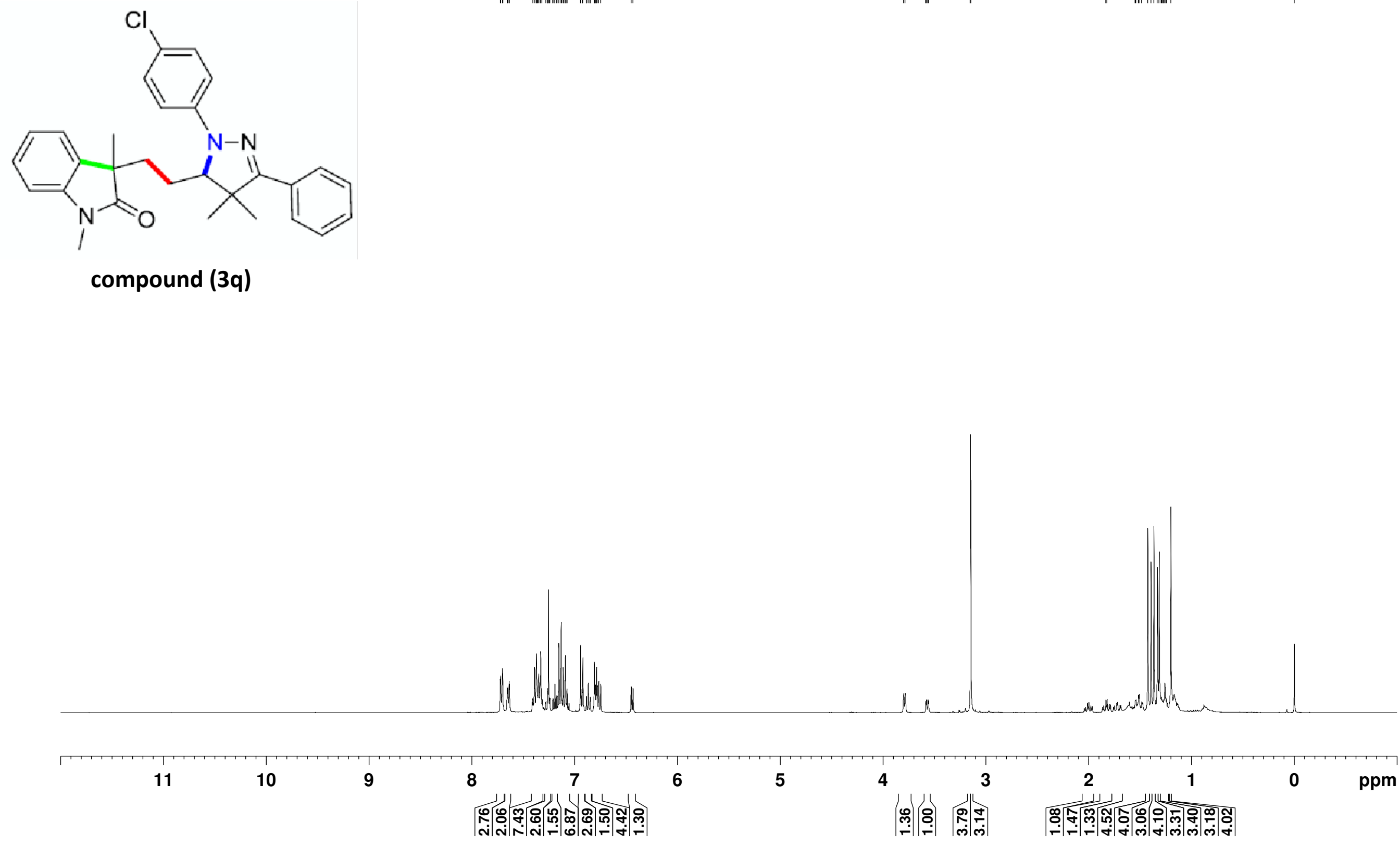

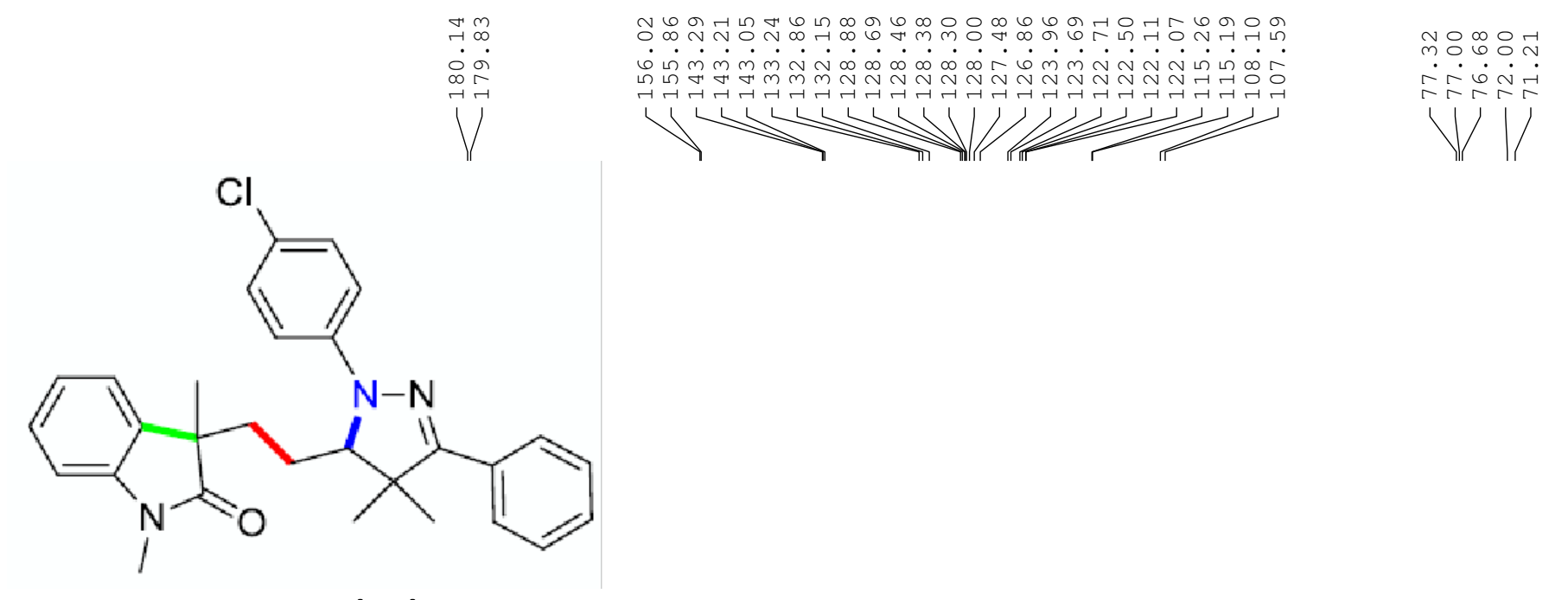

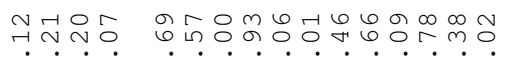

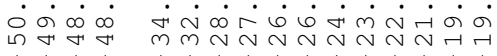
IV MWIIH

compound (3q)

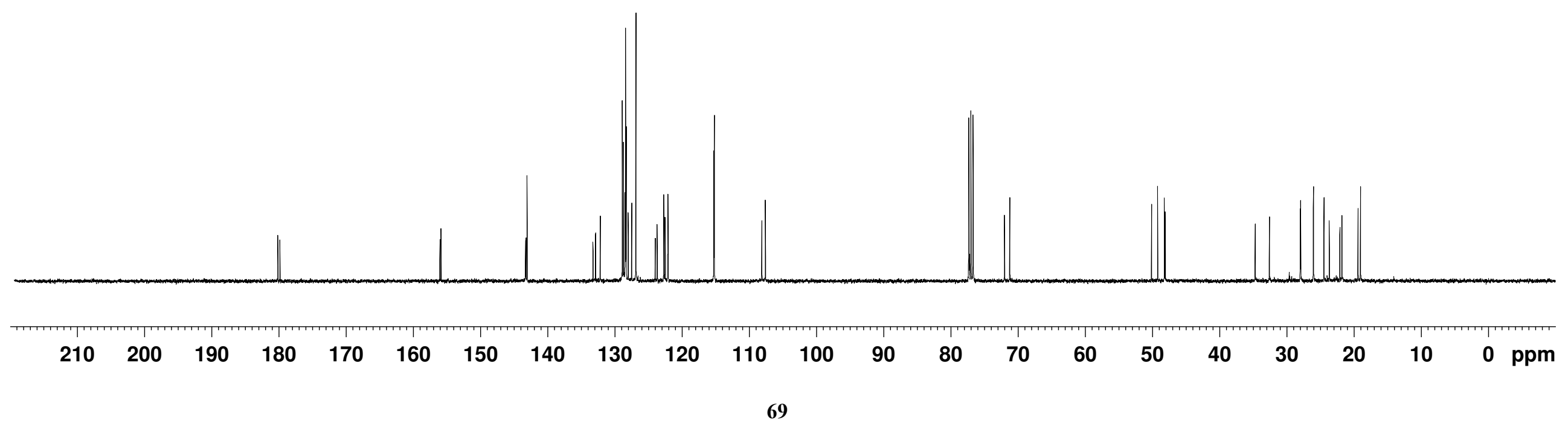




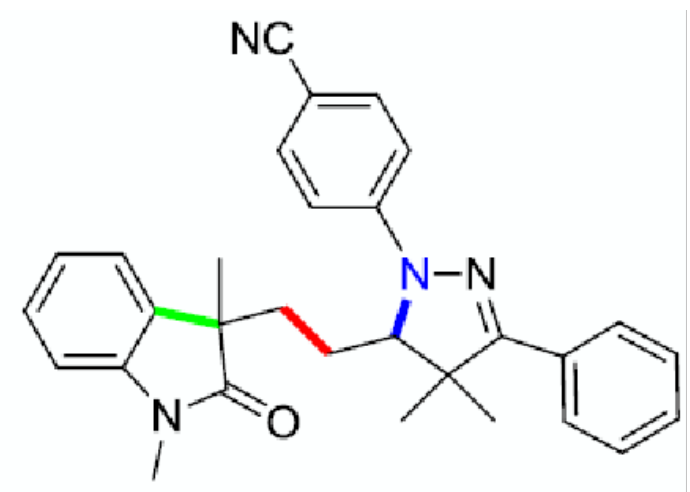

\section{compound (3r)}

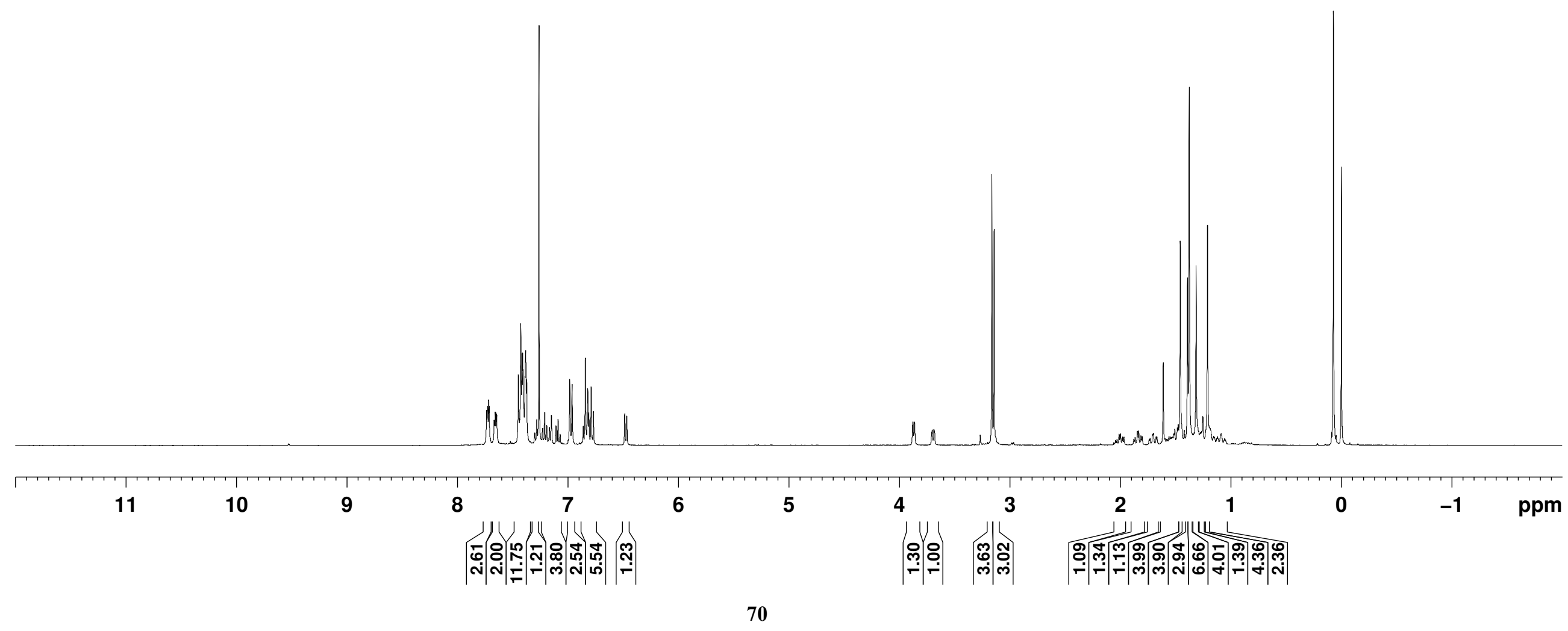



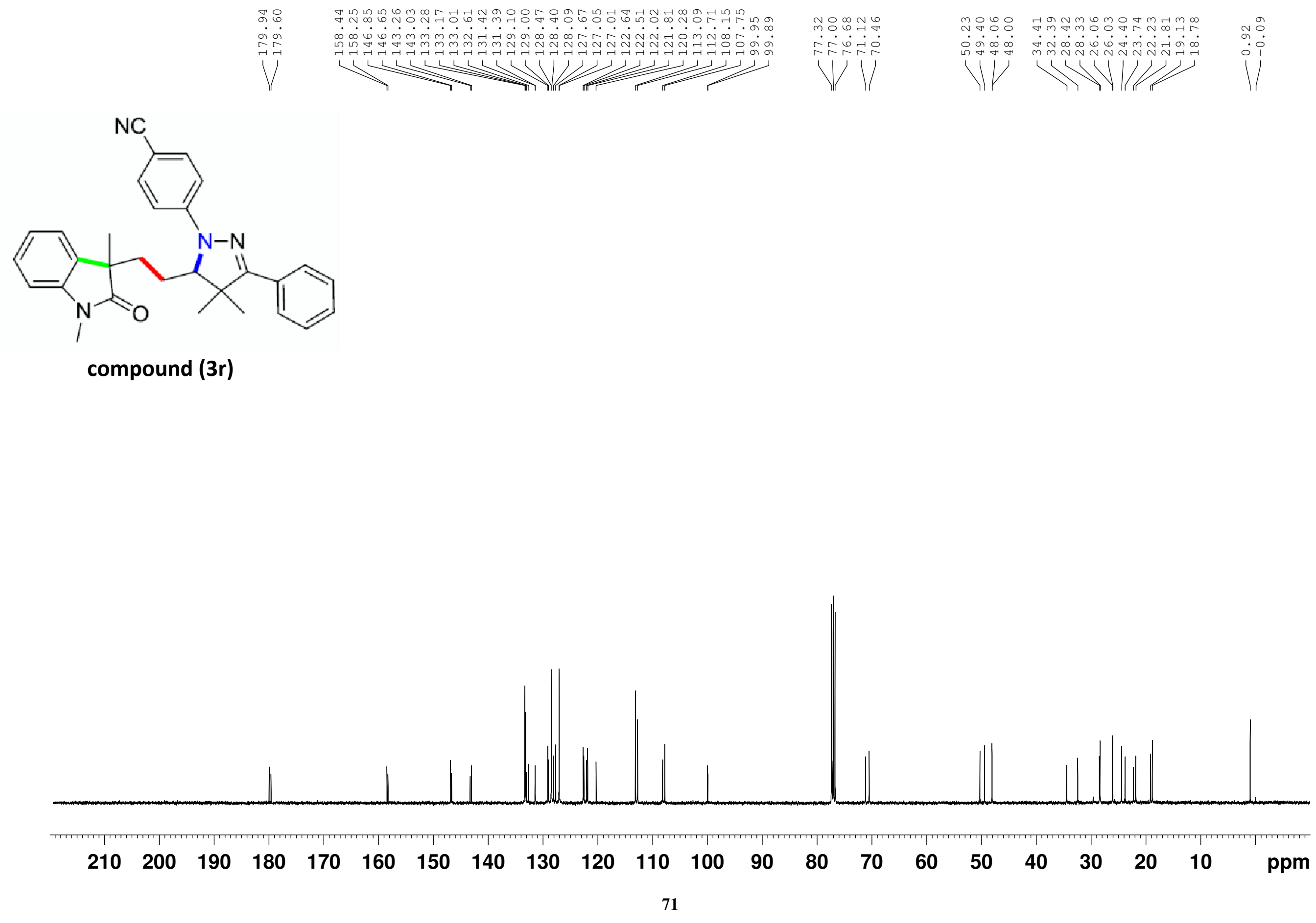

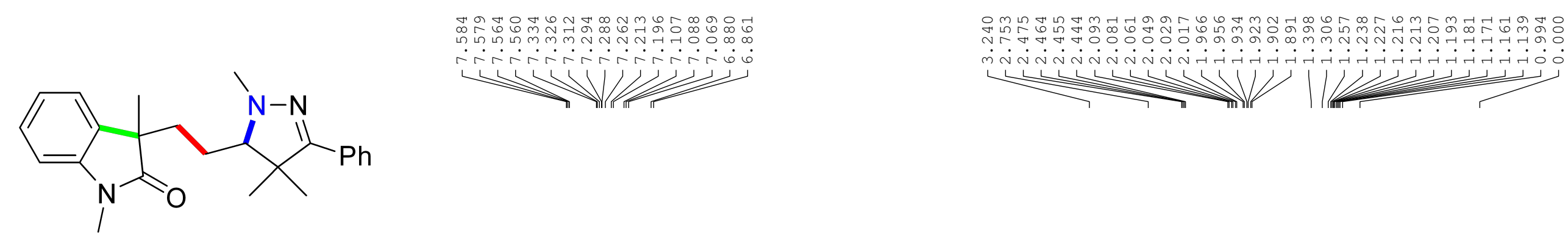

compound (3s)

major

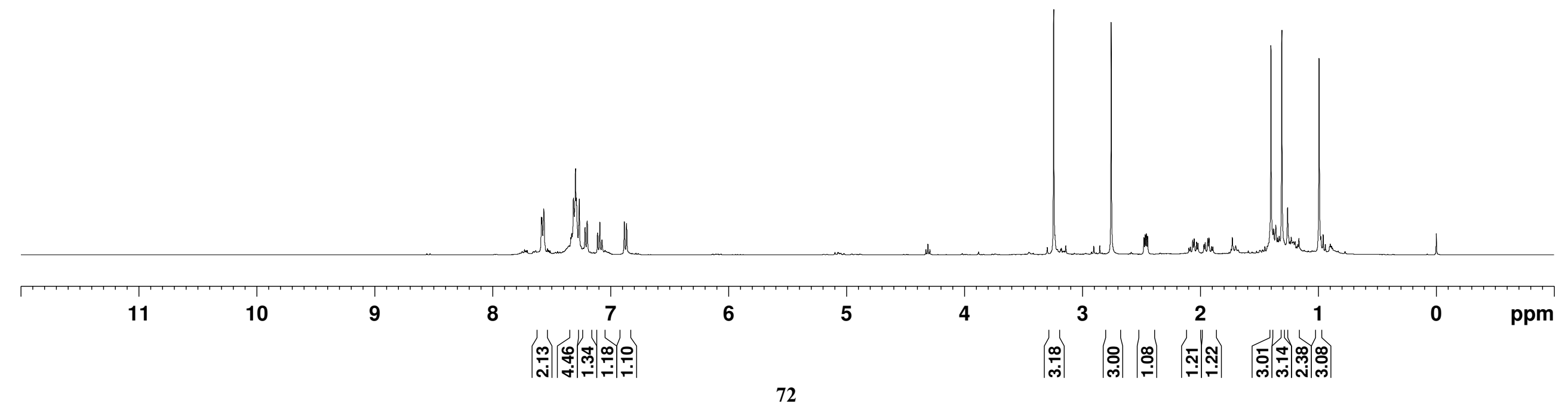



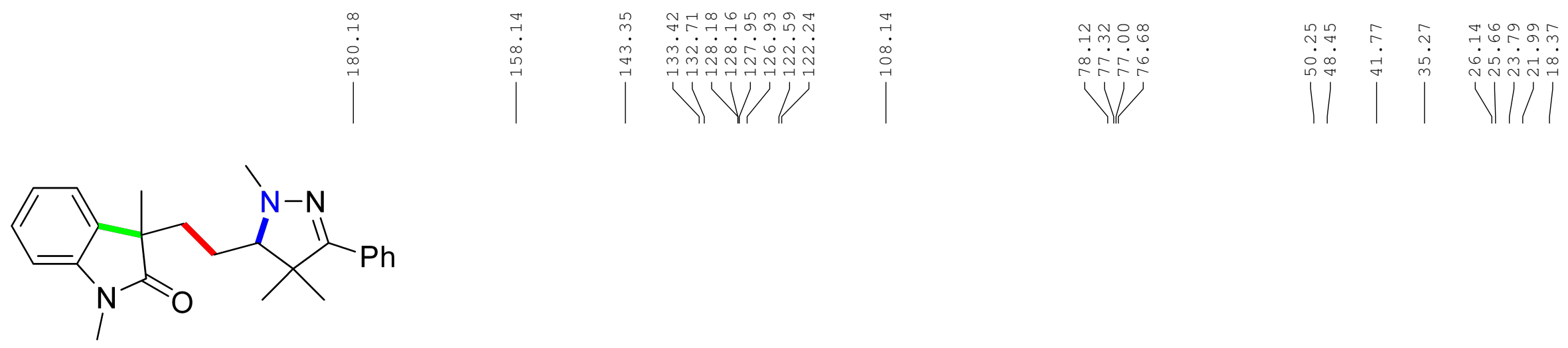

compound (3s)

major

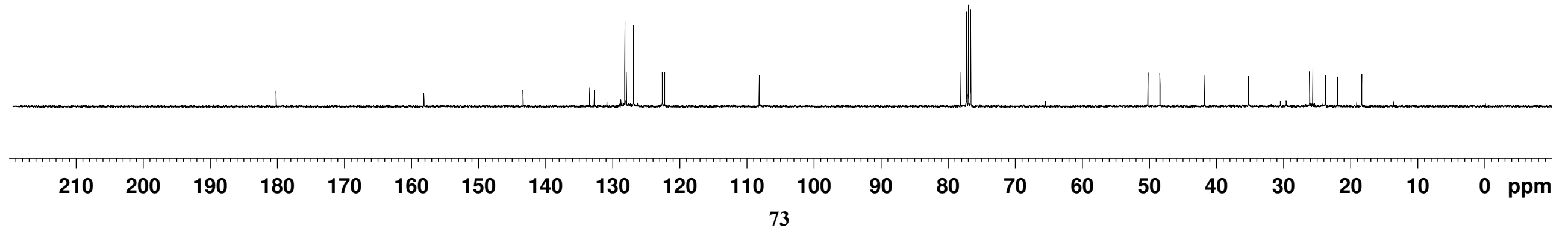




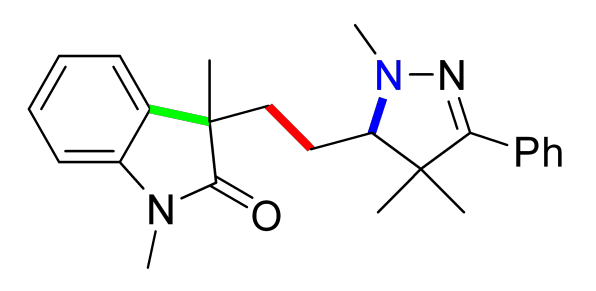

\section{compound (3s)}

minor

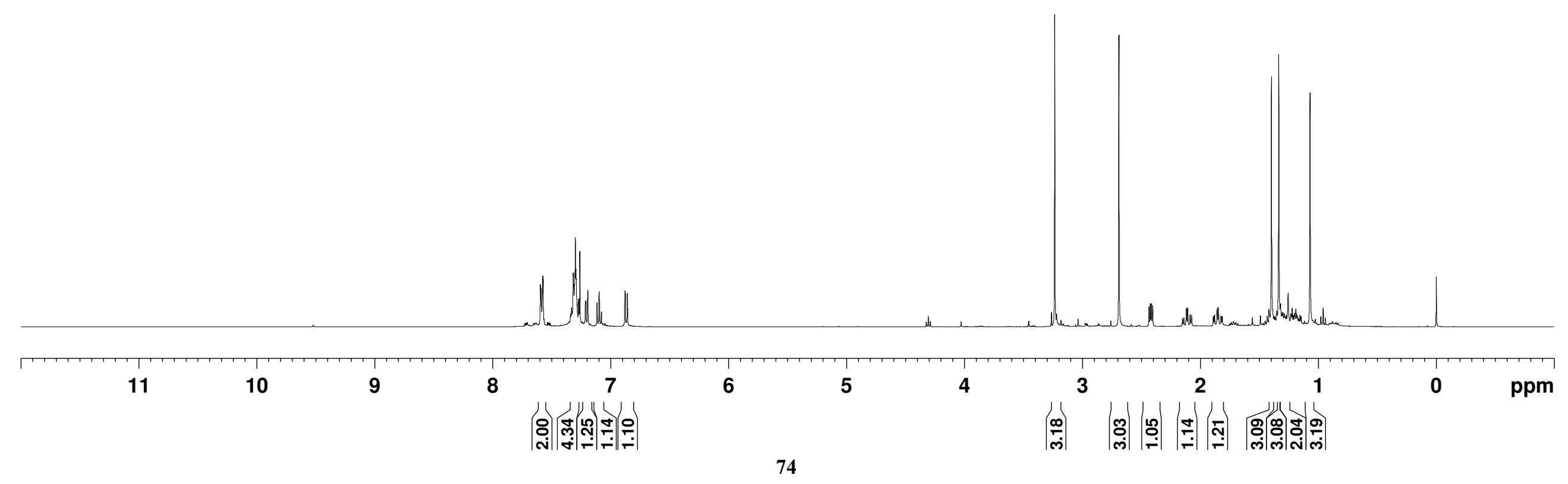



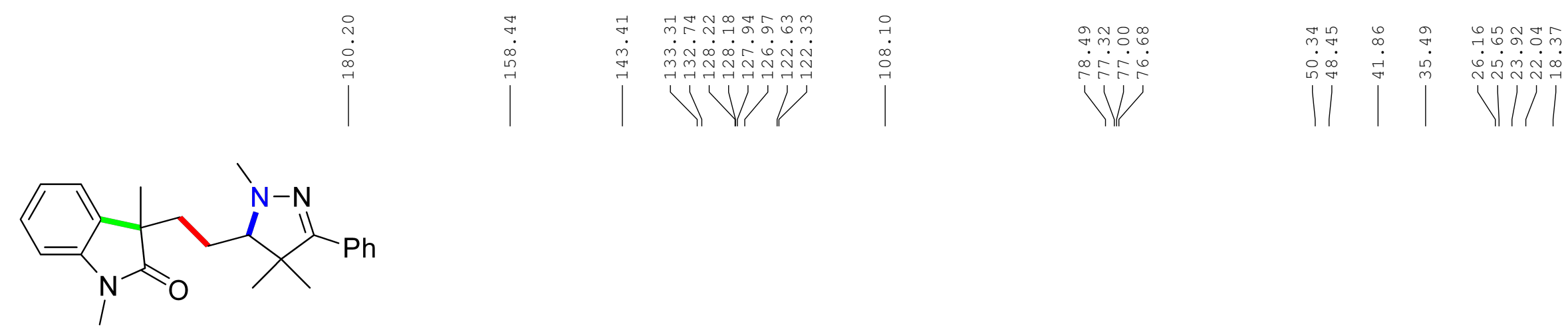

compound (3s)

minor

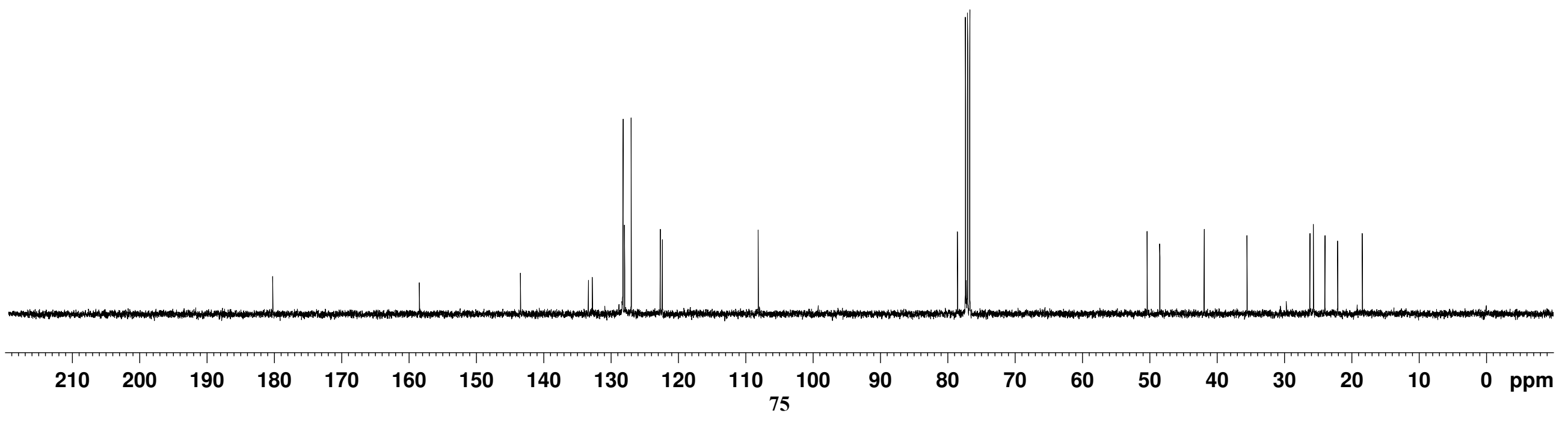




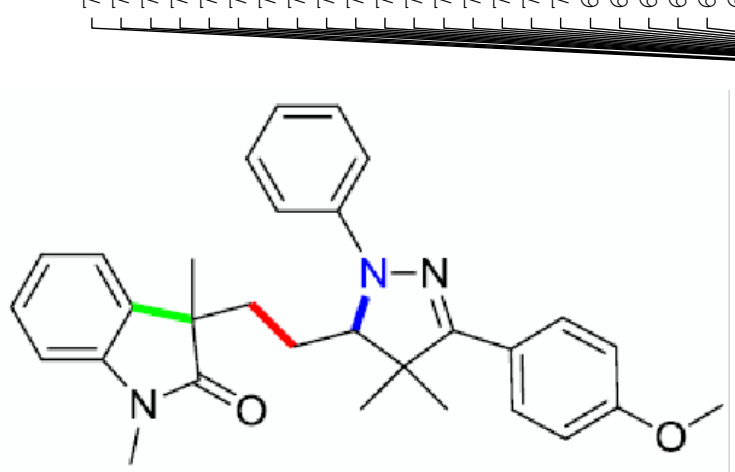

compound (3v)

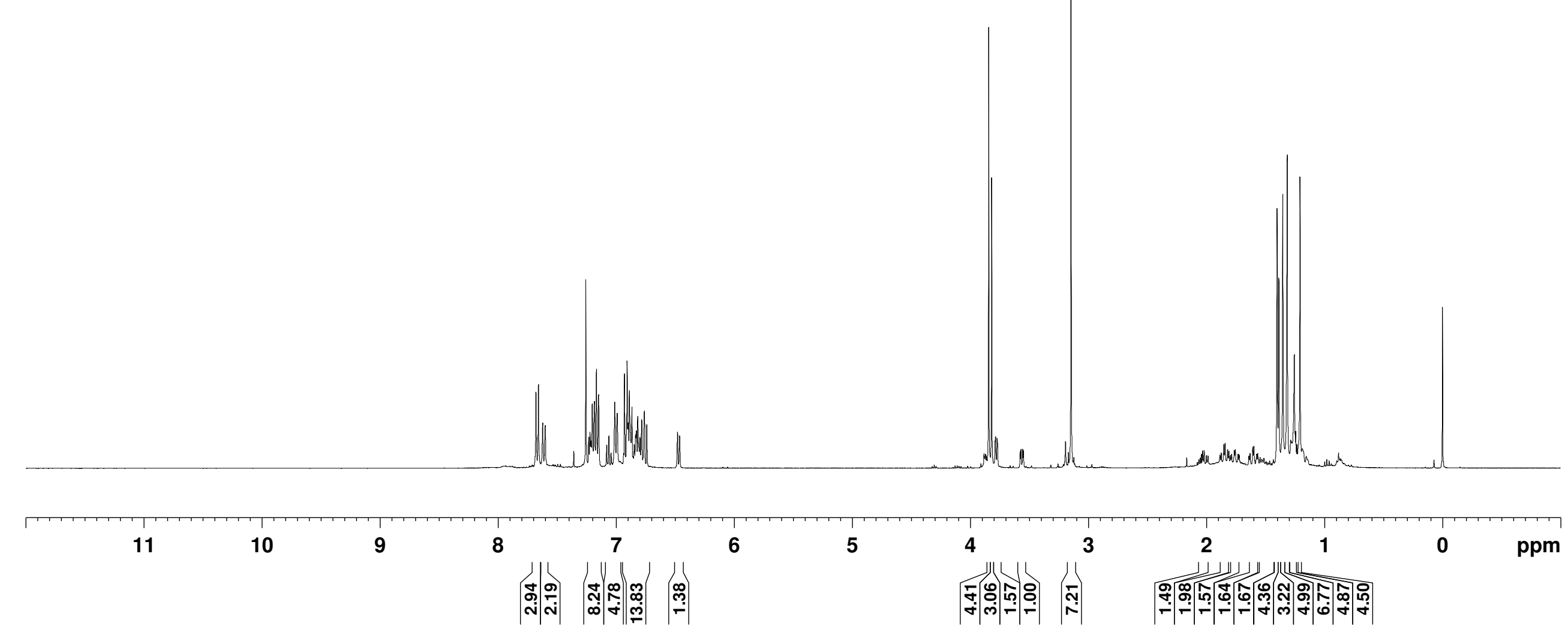



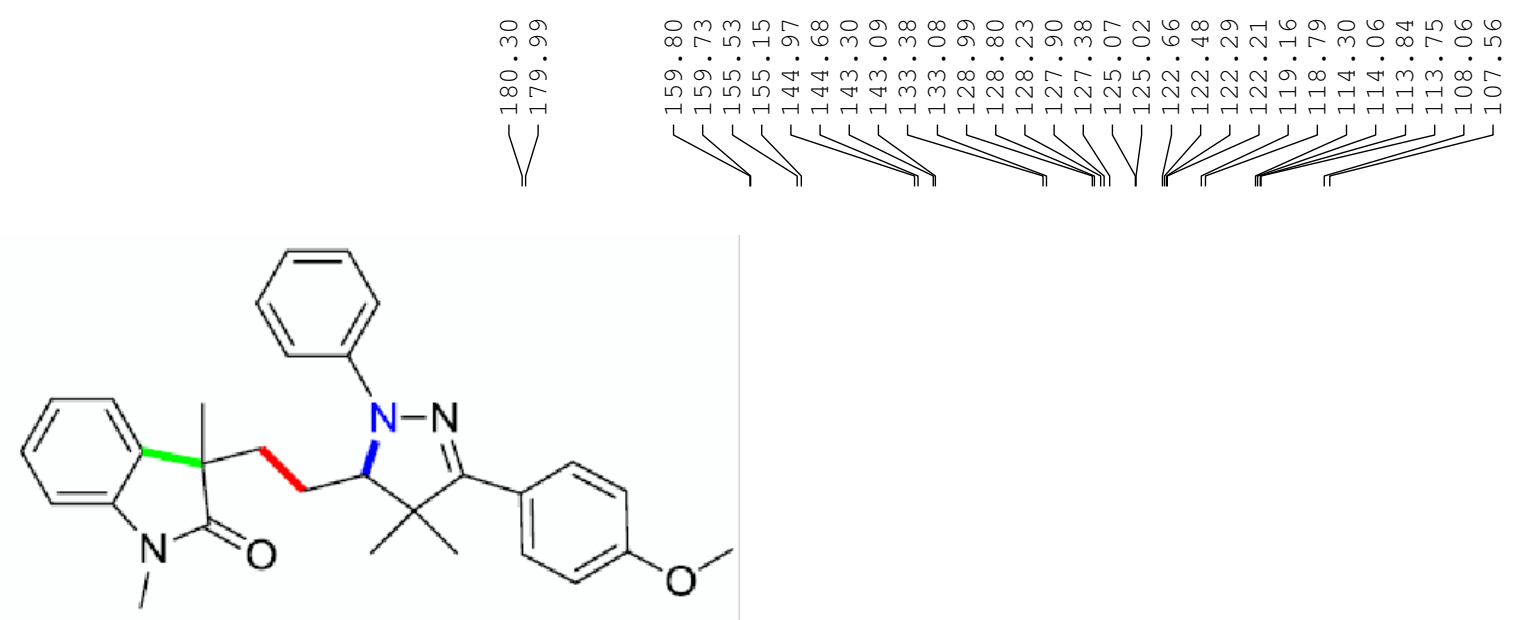

compound (3v)

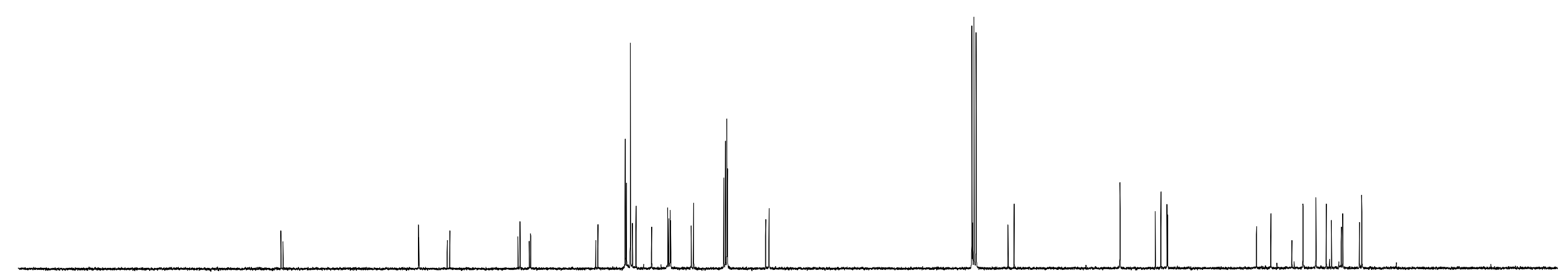

2102 

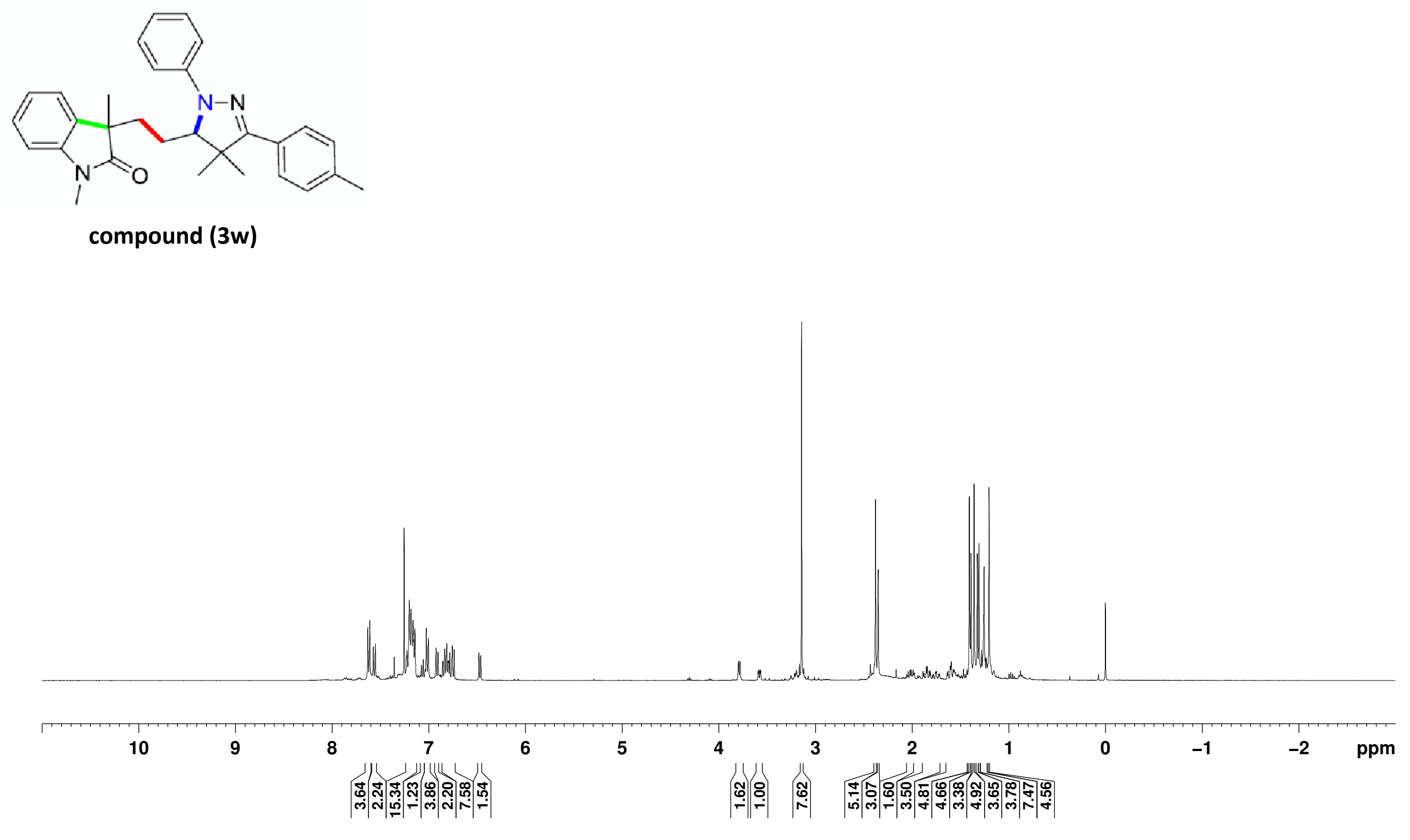


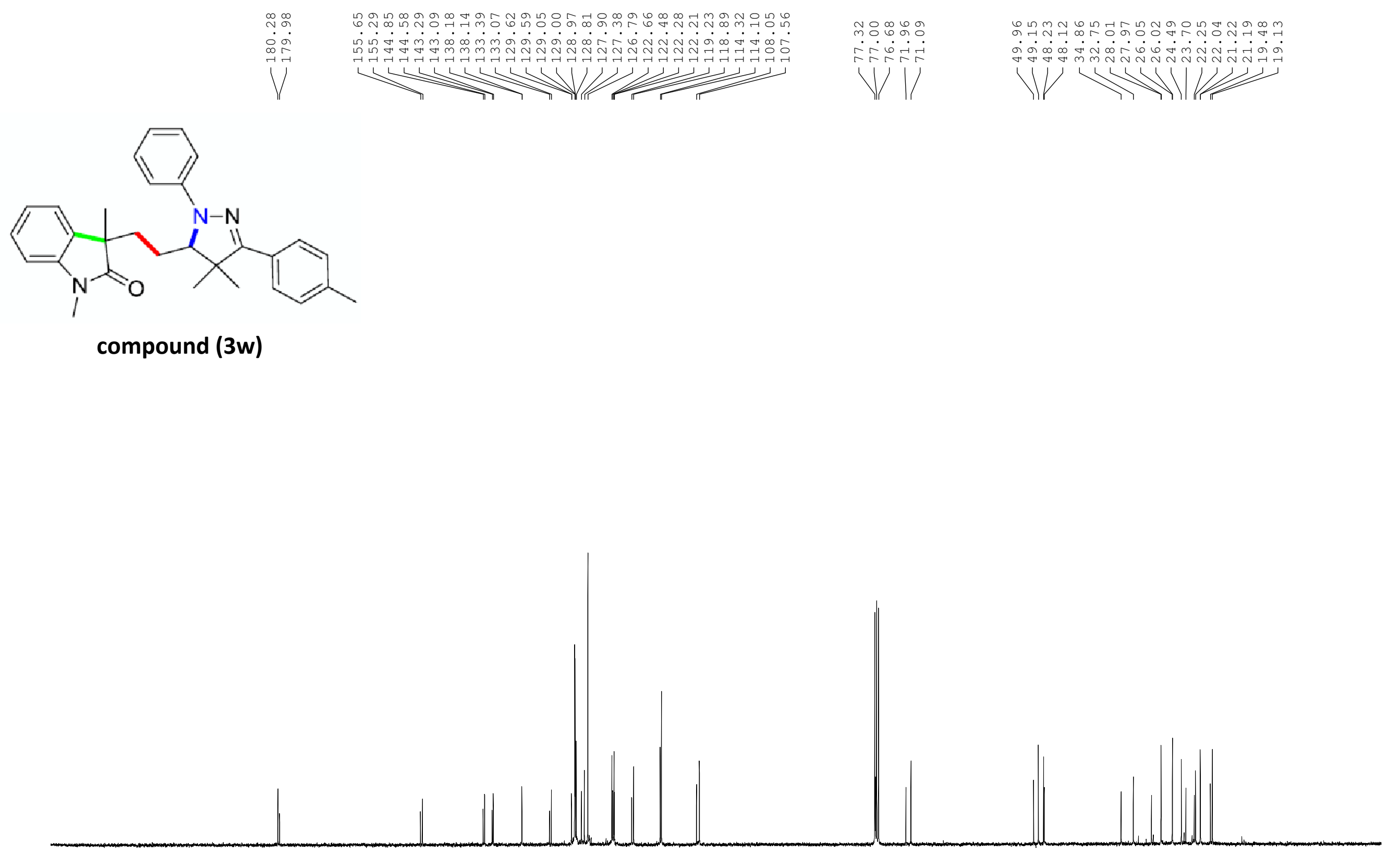

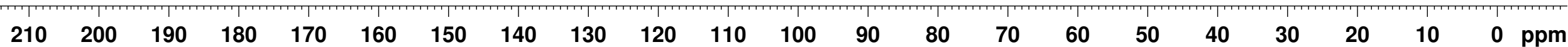




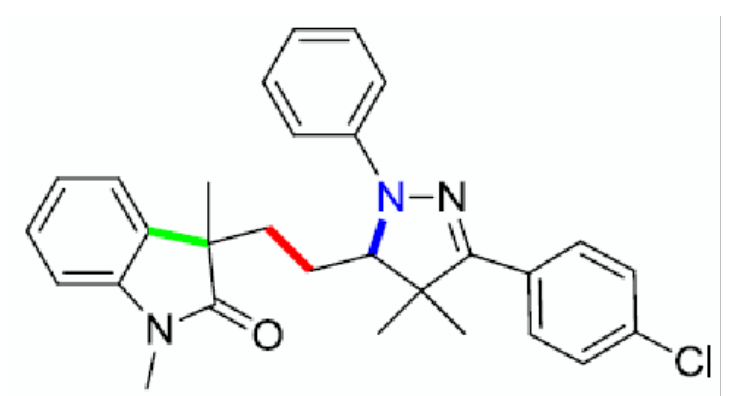

compound (3x)

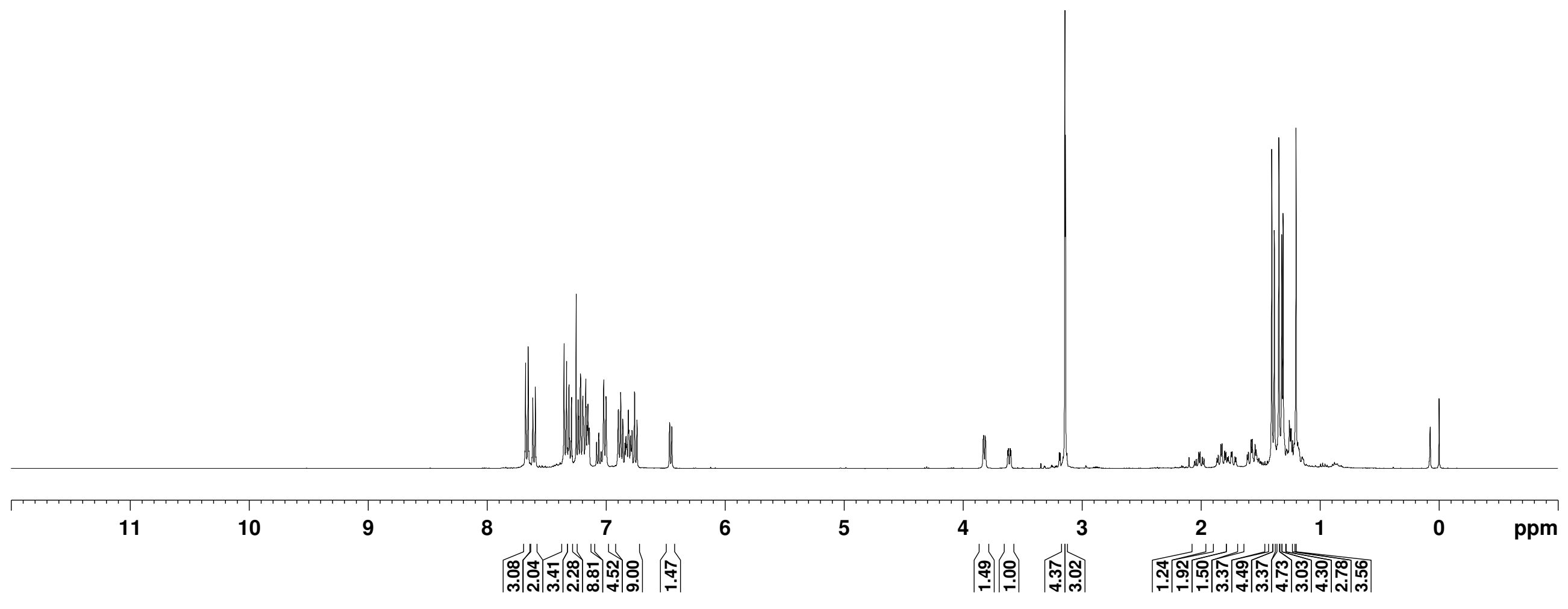



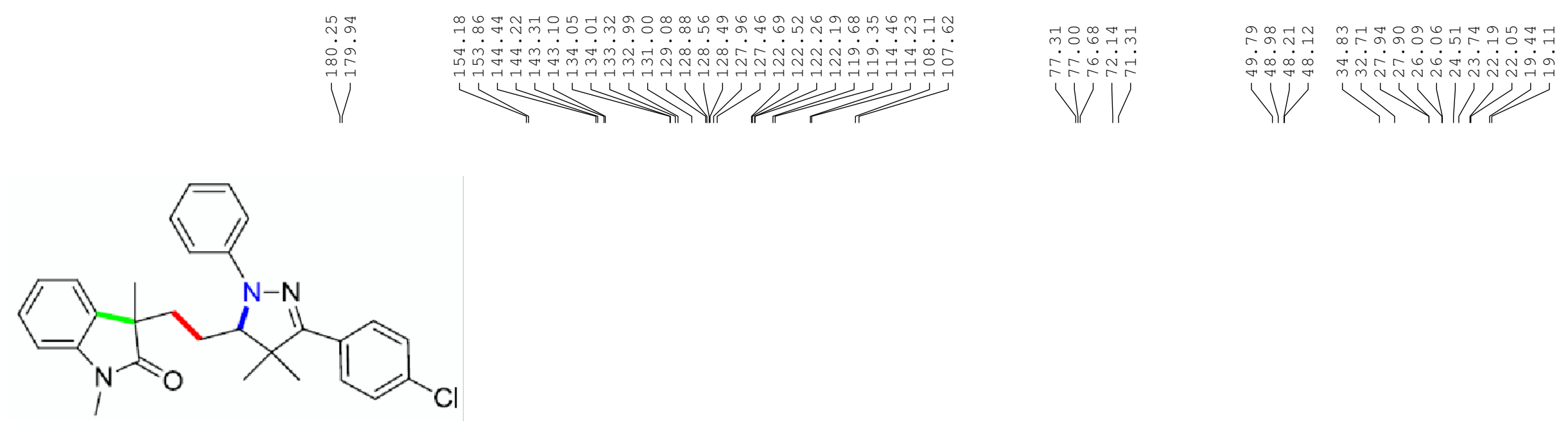

compound (3x)

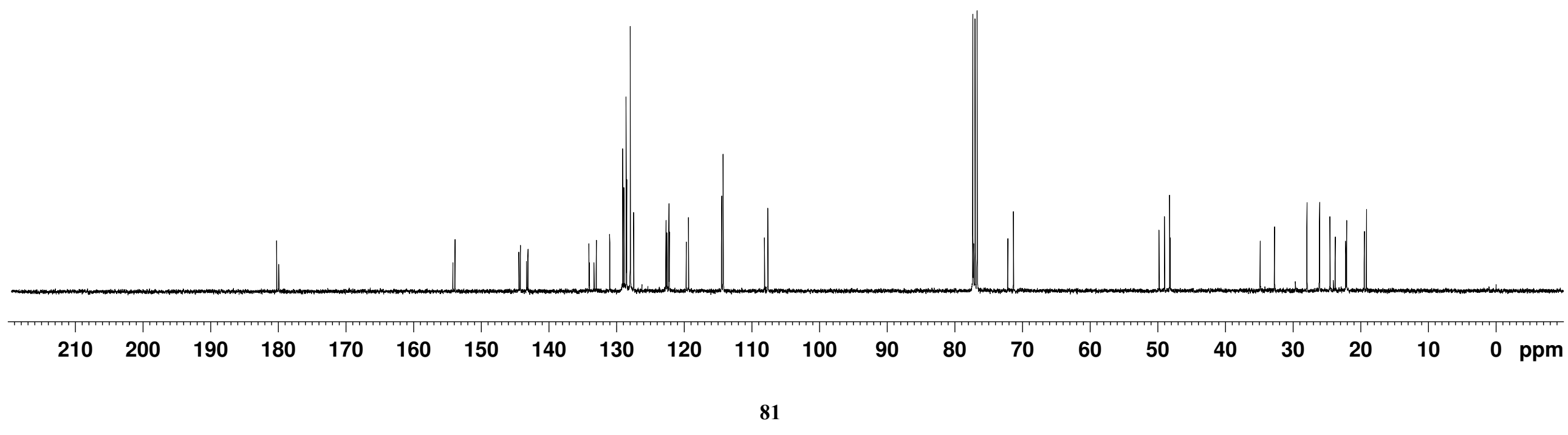




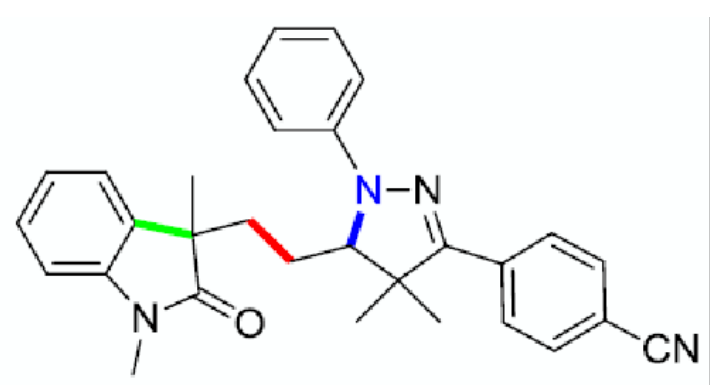

compound (3y)

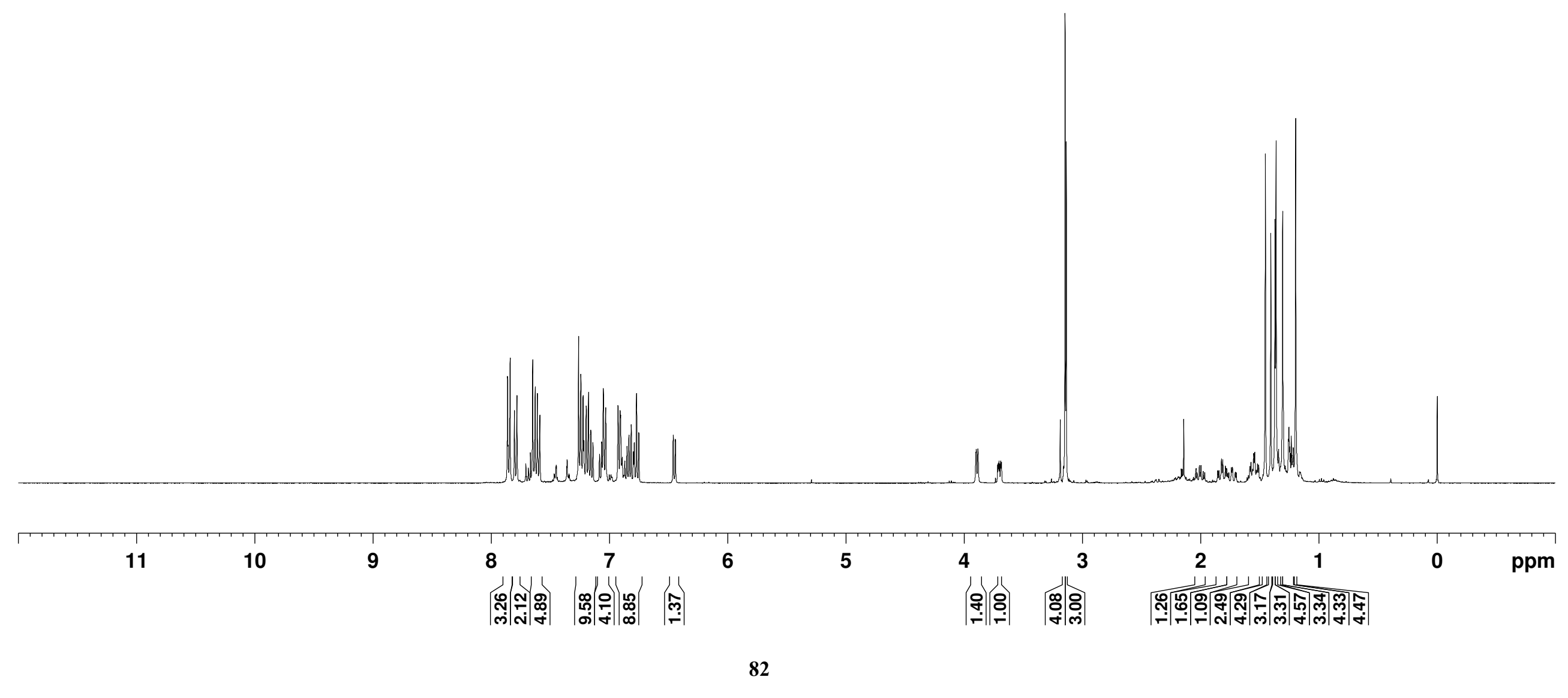



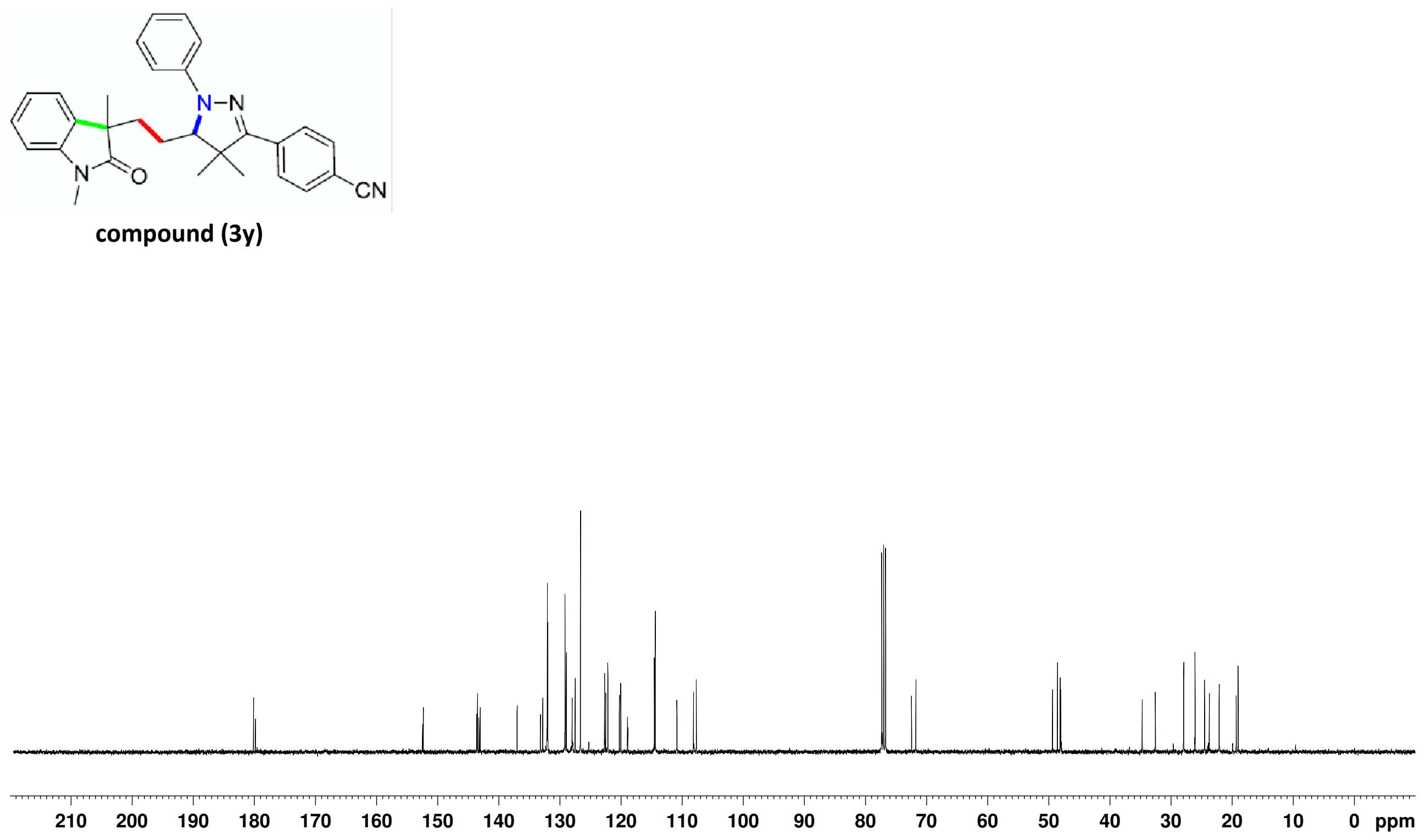


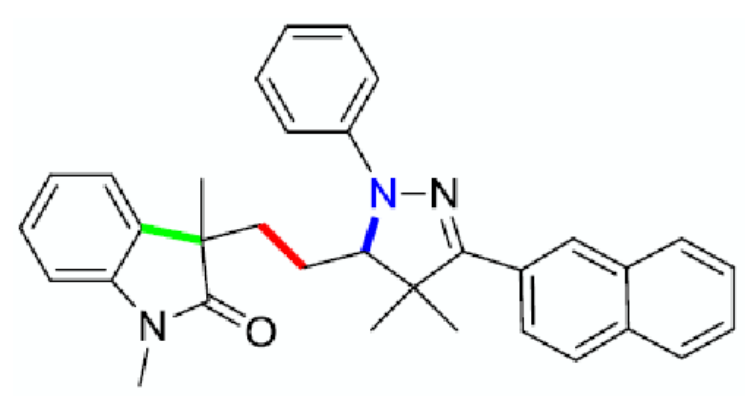

compound (3z)

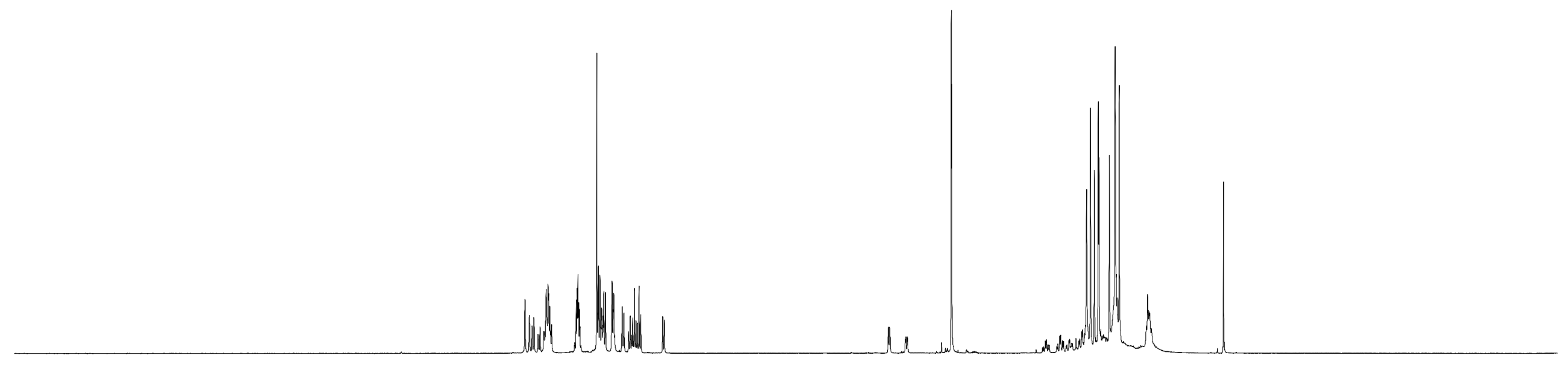




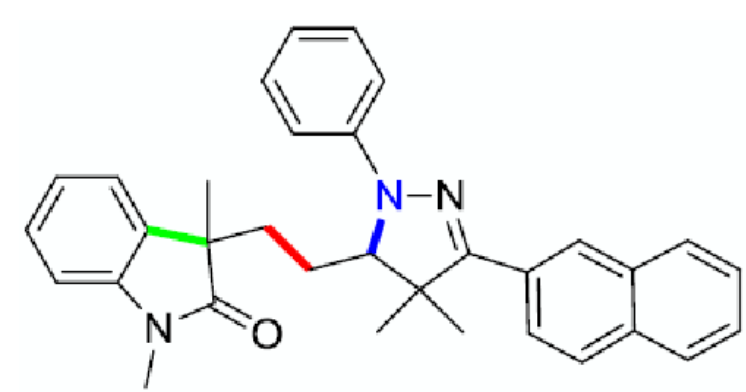

compound (3z)

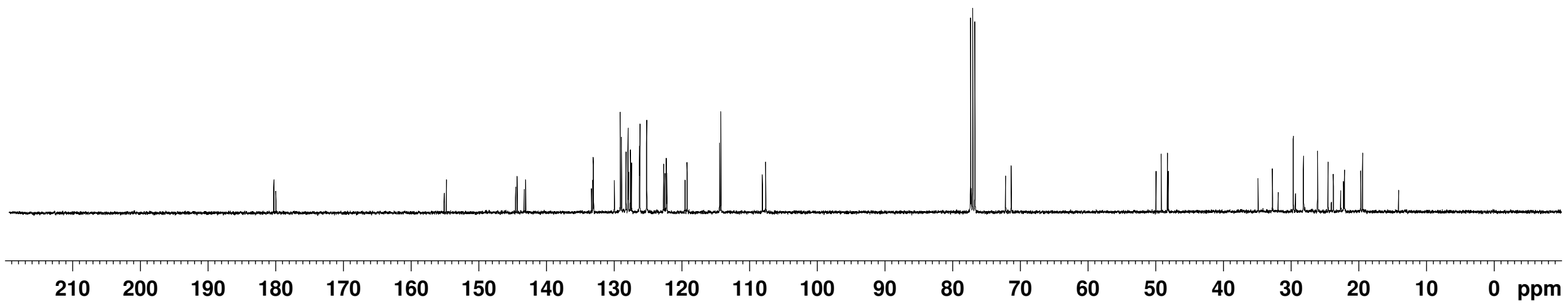



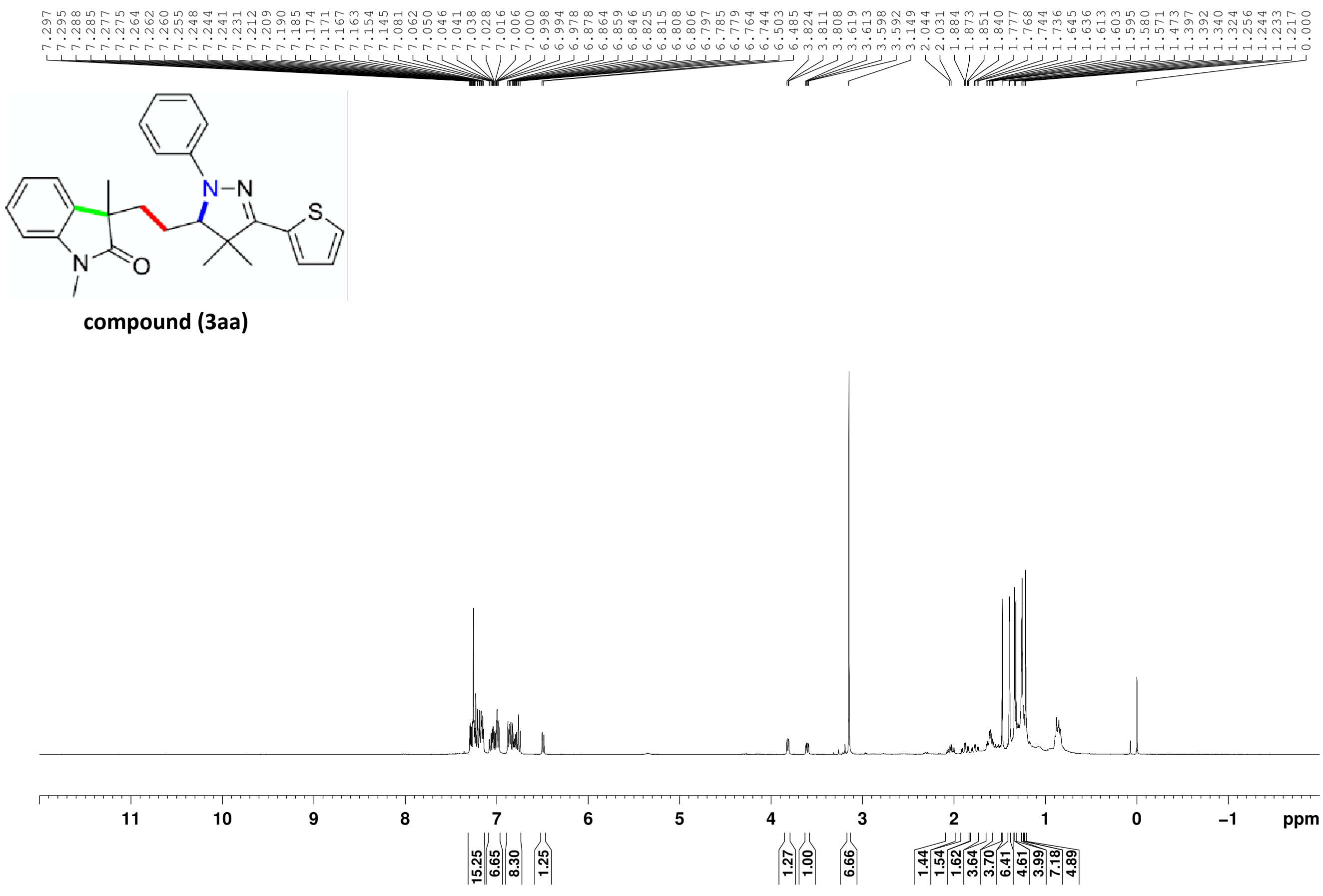


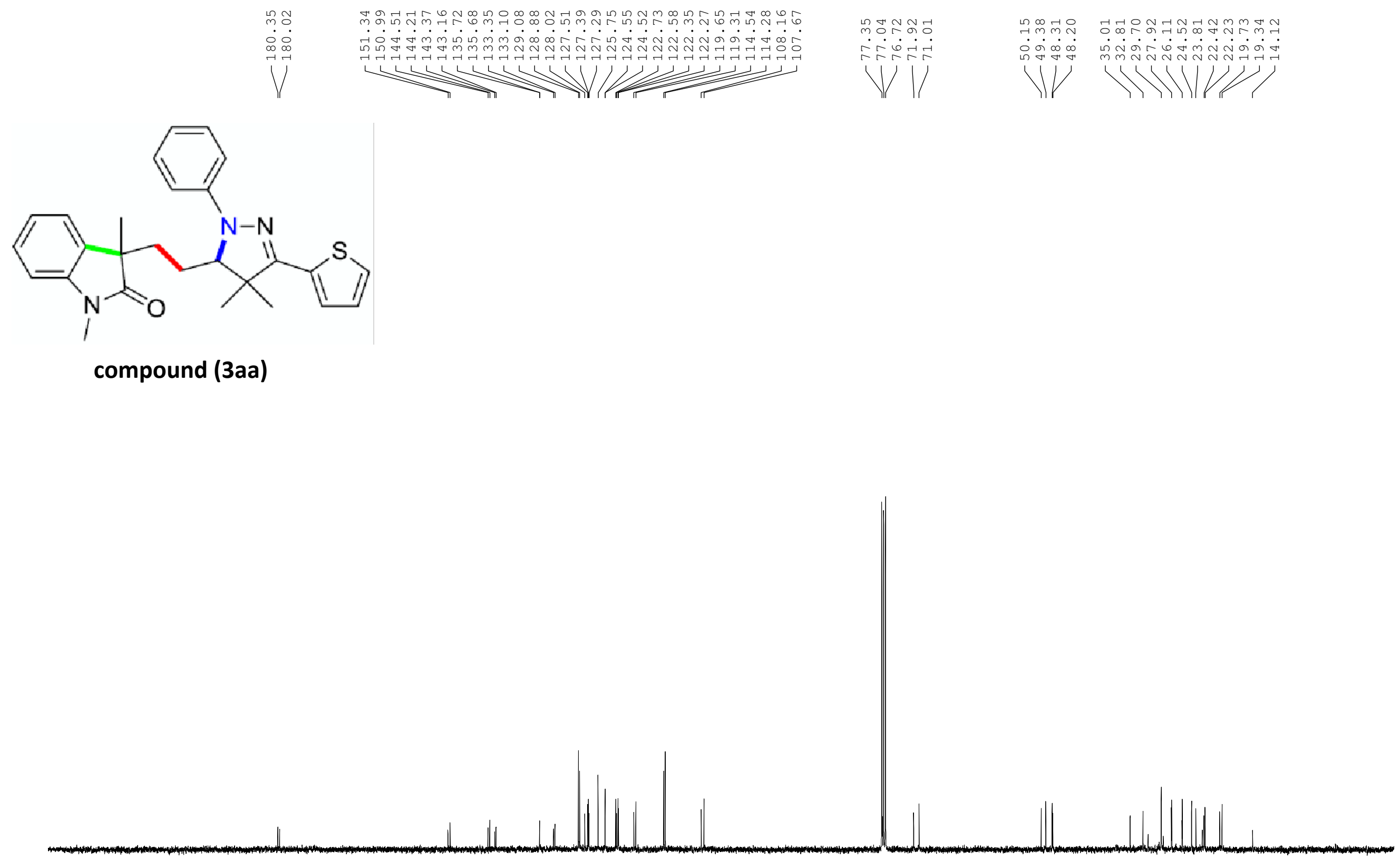

210200

190

180

170

150

$140 \quad 130$

120 

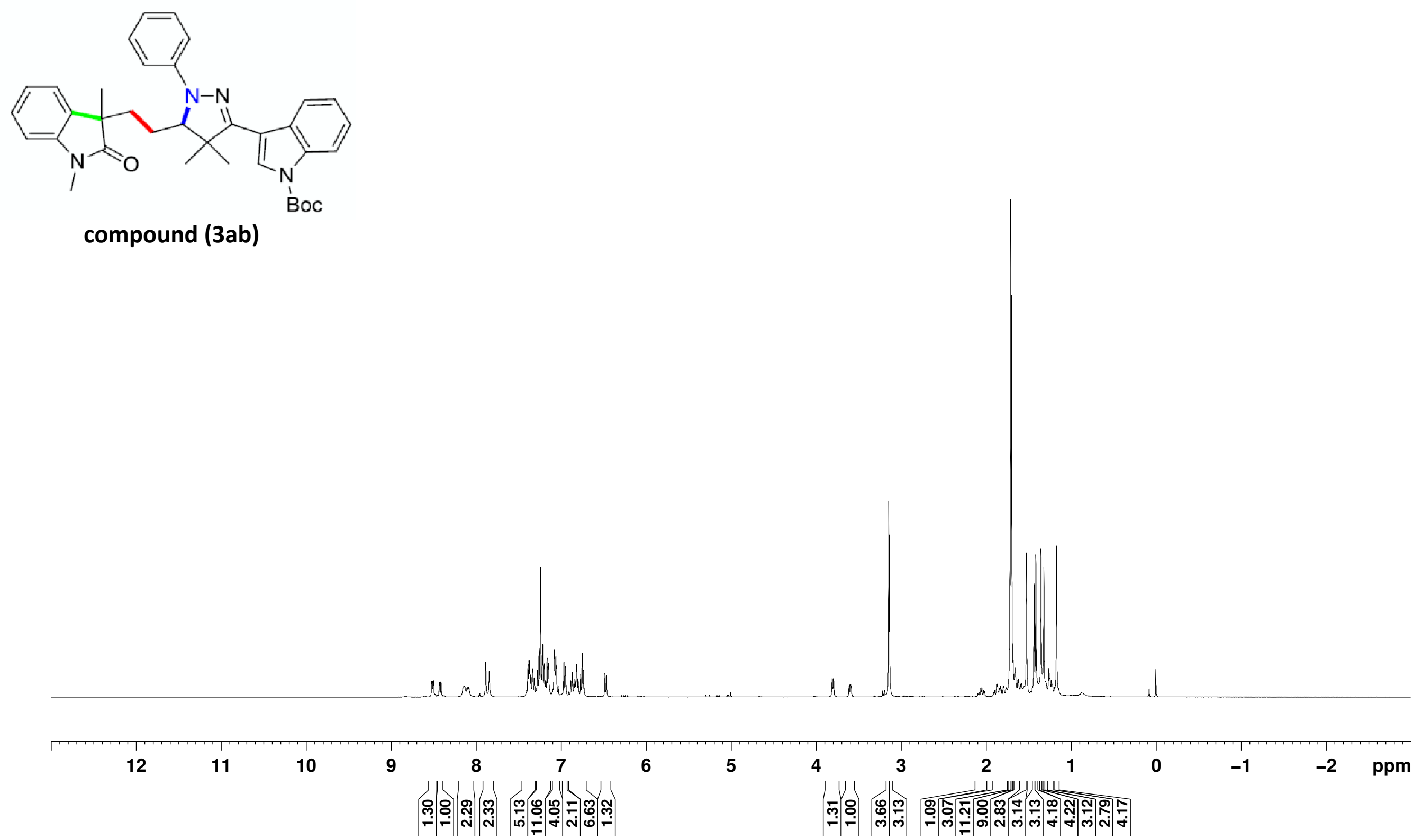

12

$11 \quad 10$

9

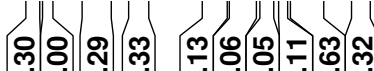

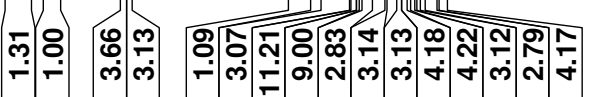




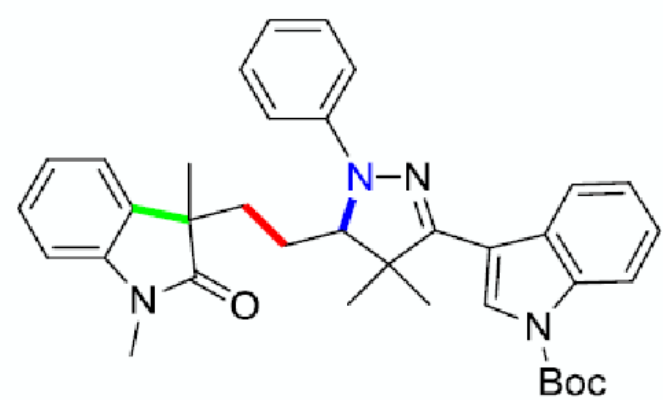

compound (3ab)

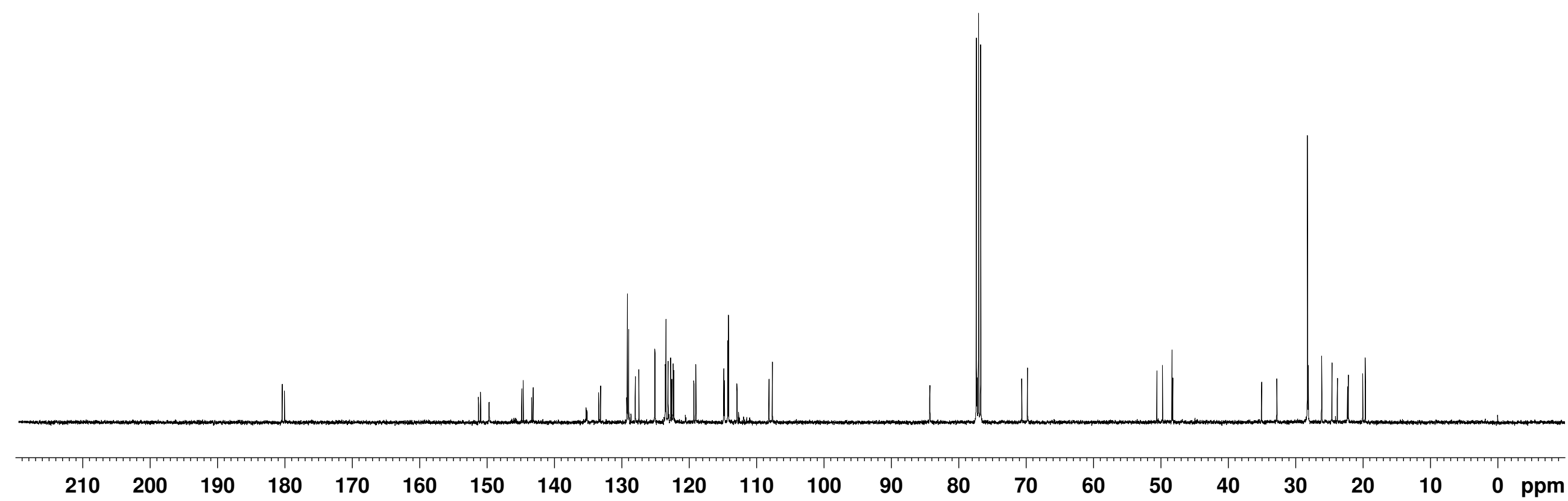




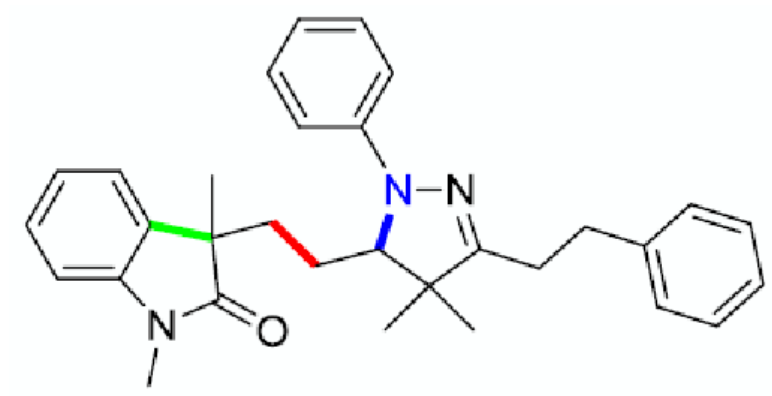

compound (3ac)

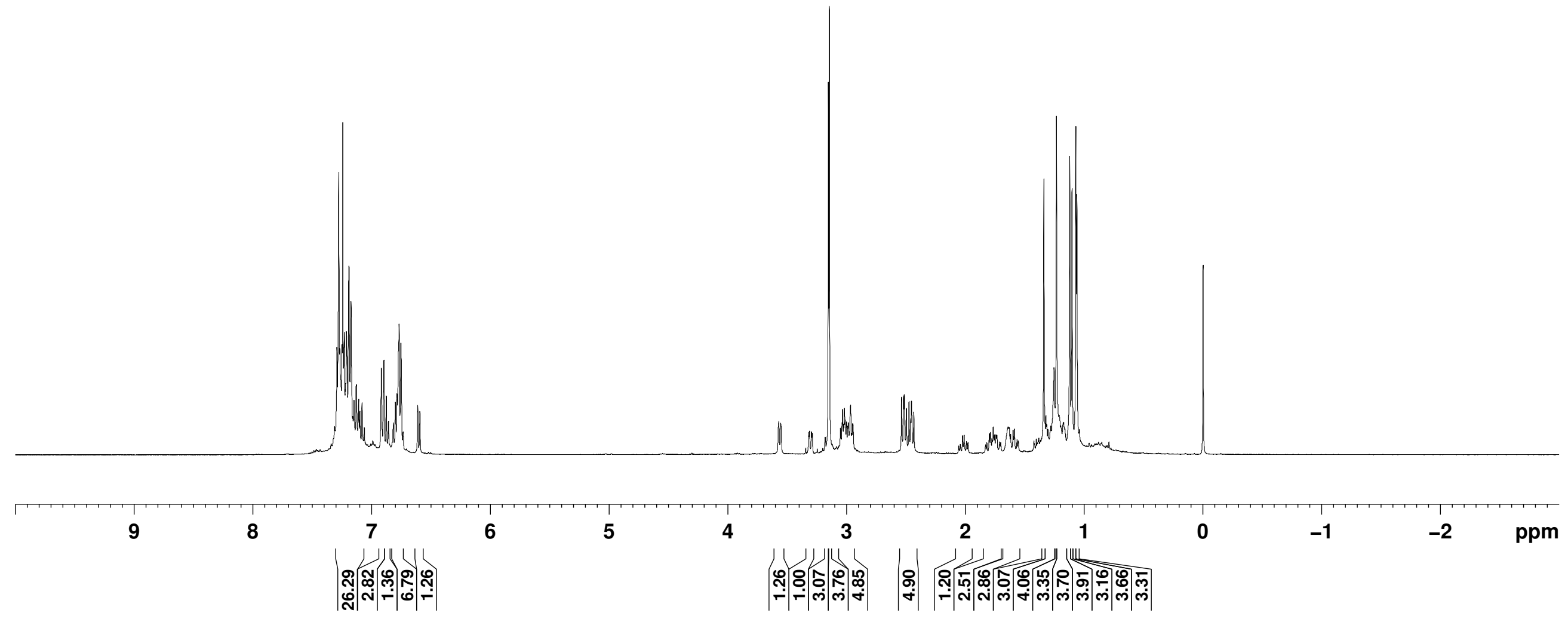



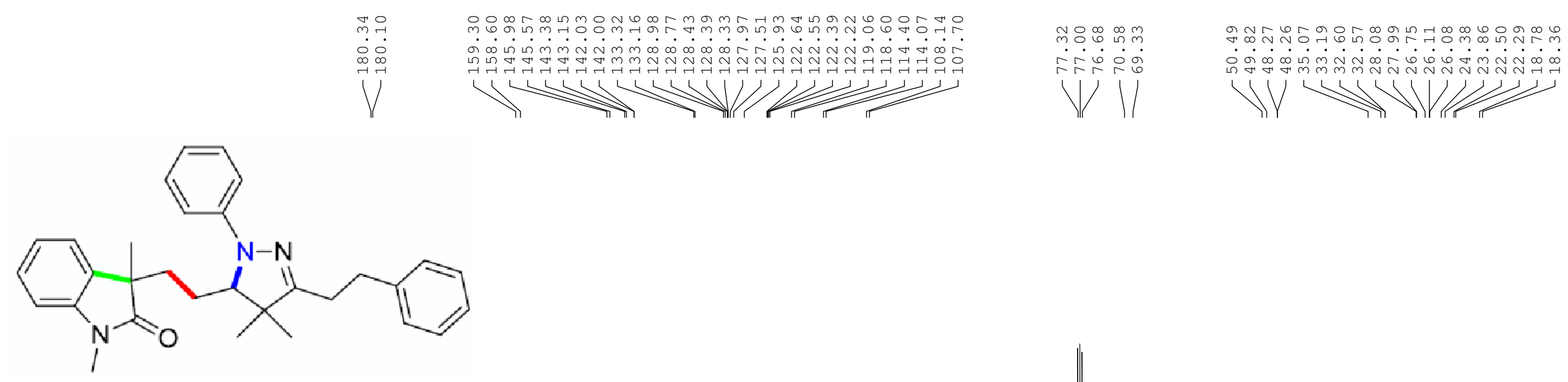

compound (3ac)

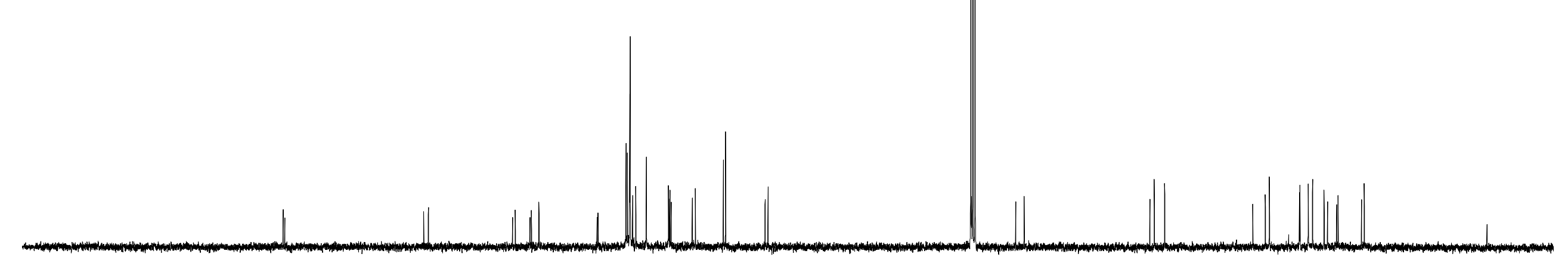




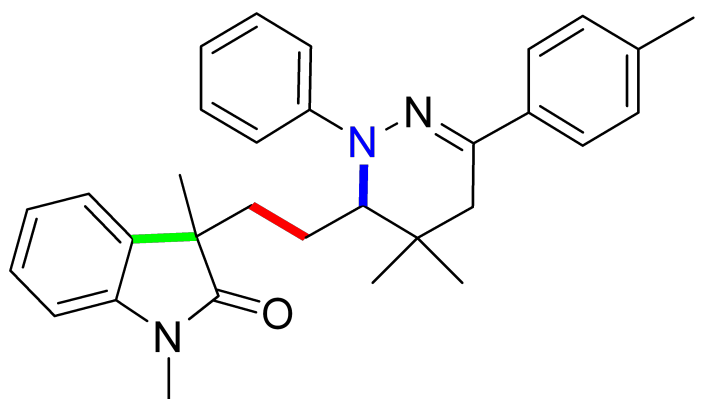

compound (5)
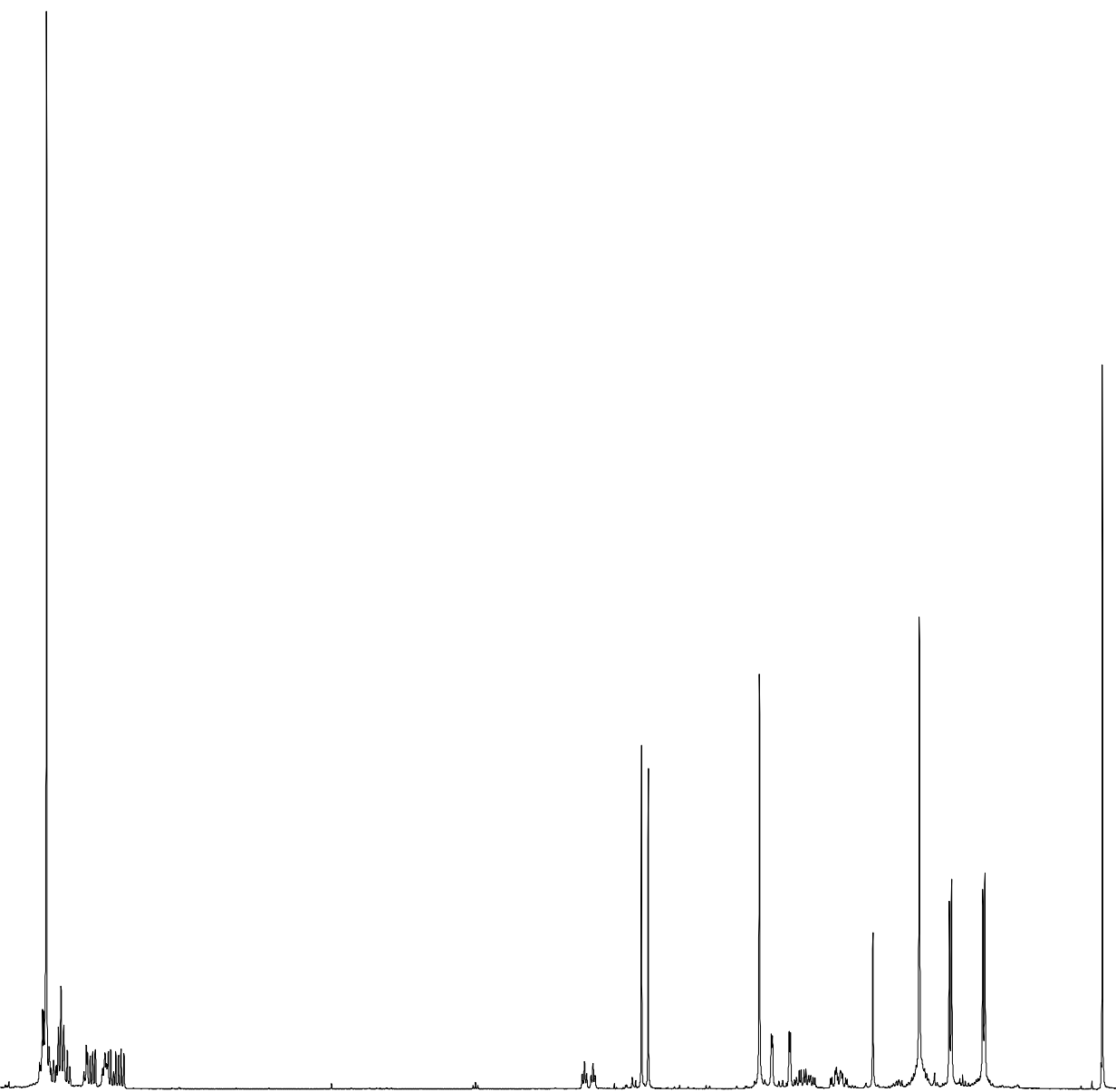

5

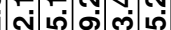
하잉
2
1

4

$\begin{array}{lll}0 & -1 & \text { ppm }\end{array}$ 


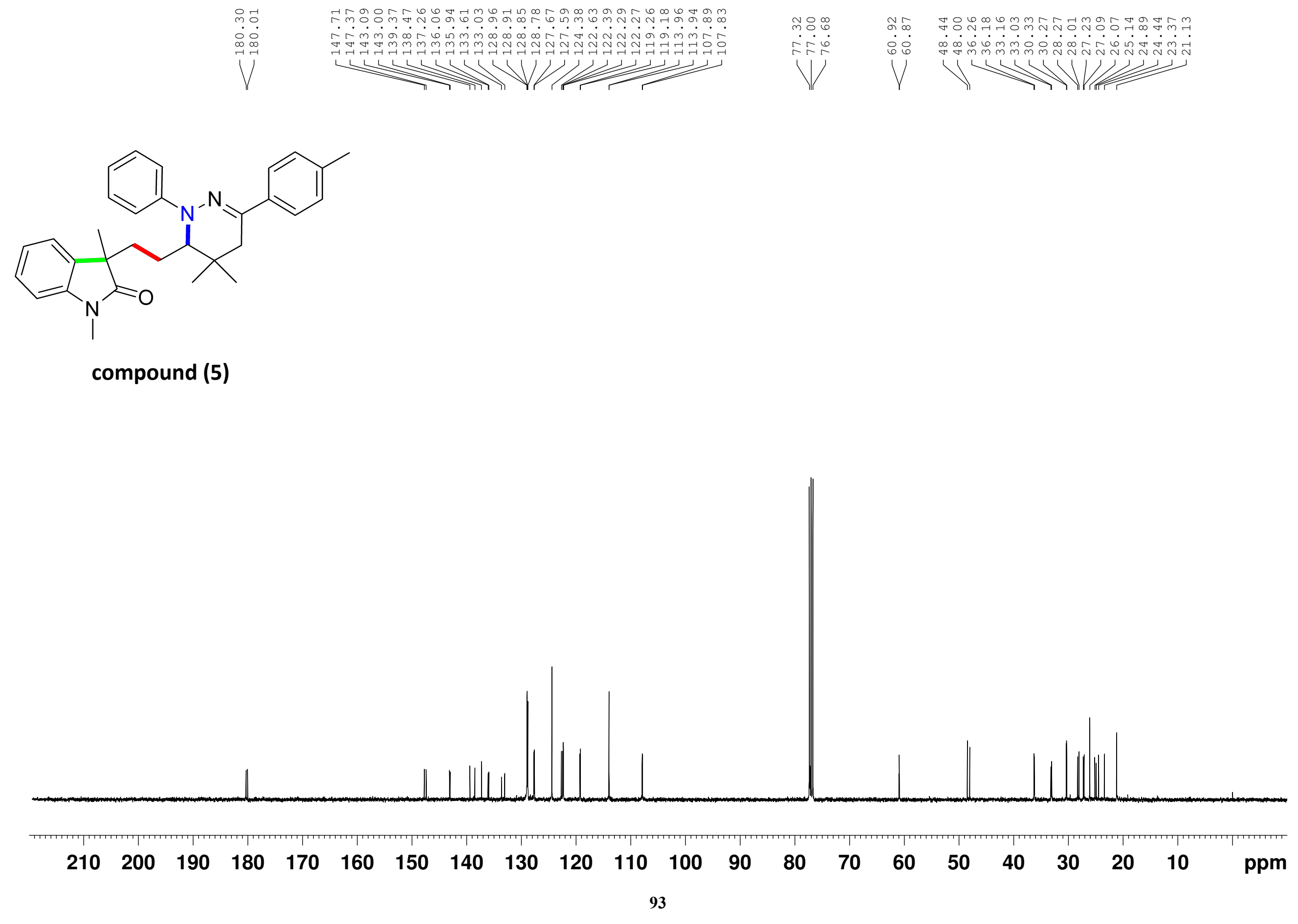

